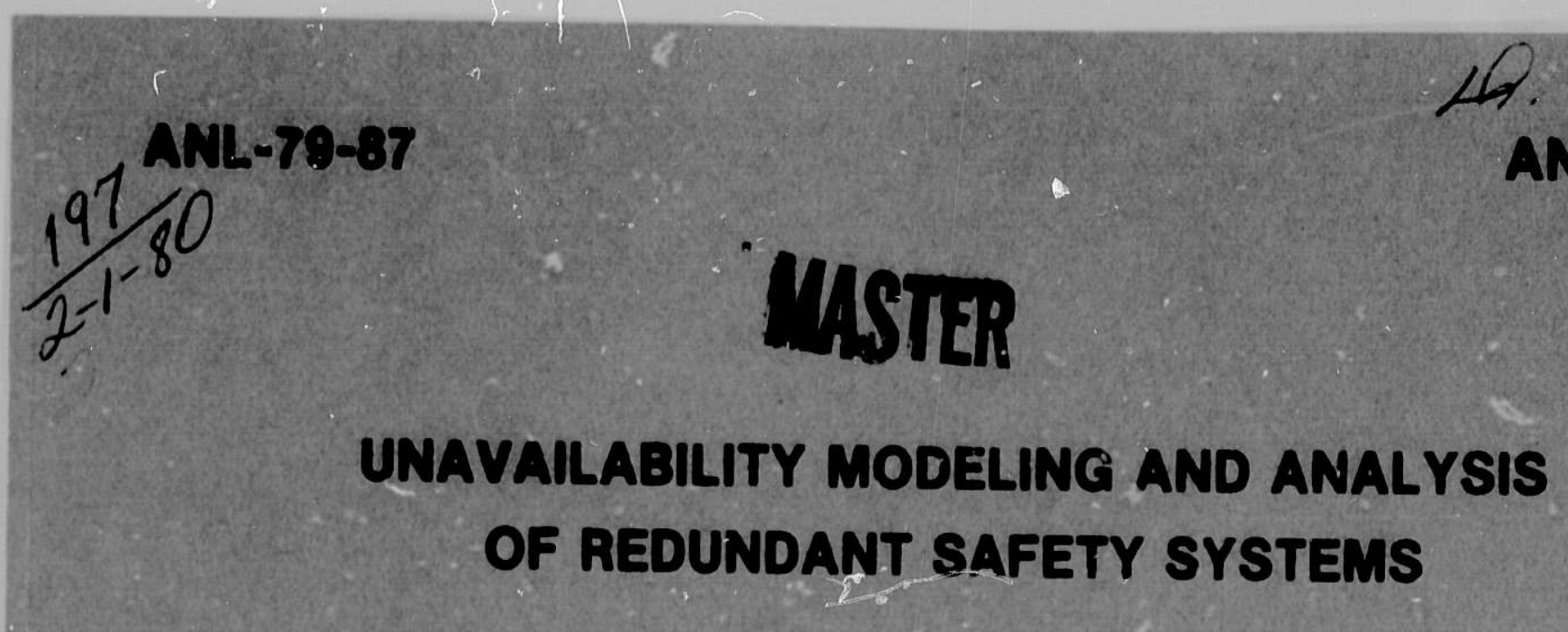

by

Jusel K. Vaurio and Dominic Sclaudone

y

3

BASE TECHNOLOGY

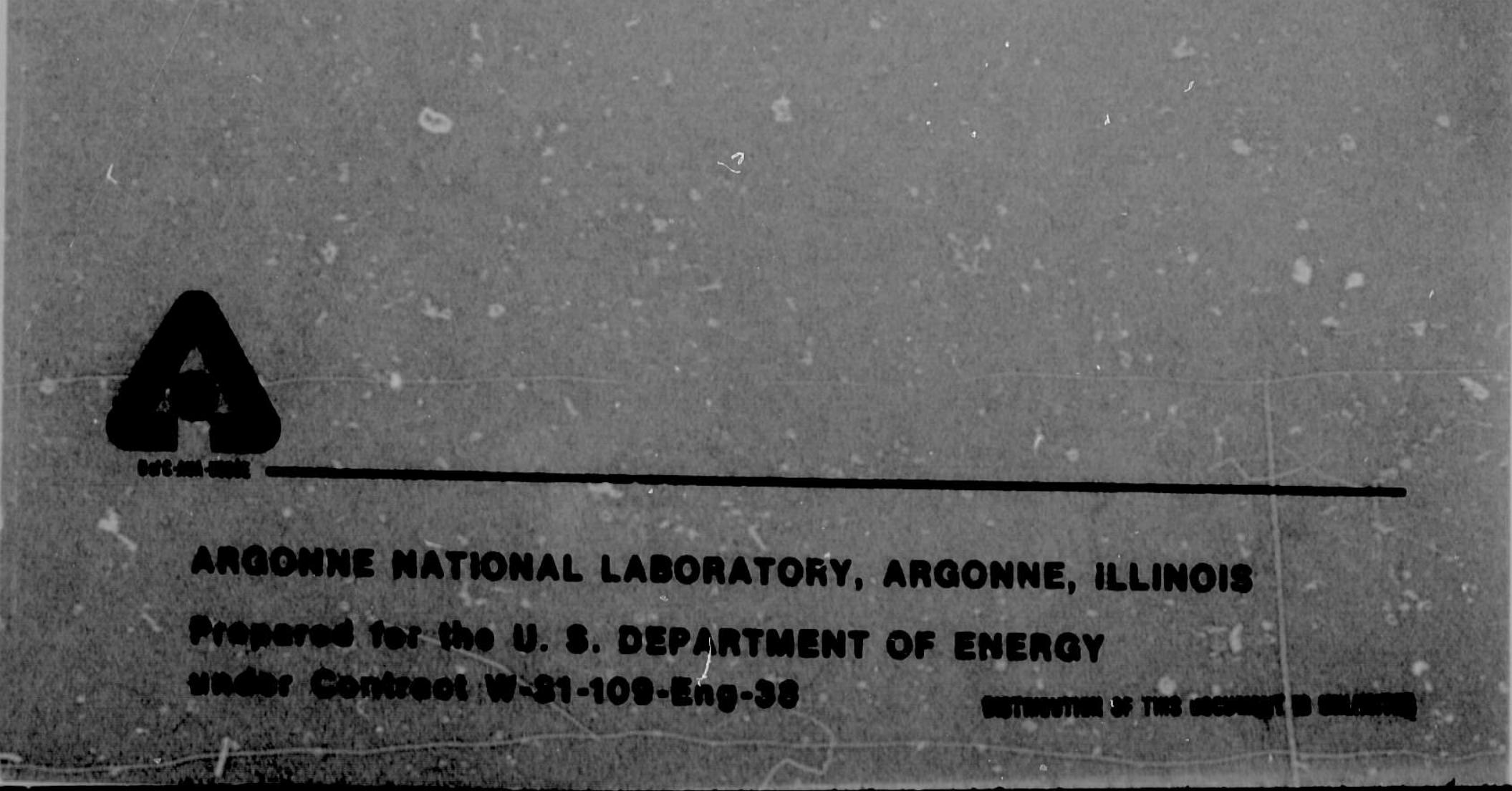


ANL-79-87

ARGONNE NATIONAL LABORATORY

9700 South Cass Avenue

Argonne, Iliinois 60439

UNAVAILABILITY MODELING AND ANALYSIS

OF REDUNDANT SAFETY SYSTEMS

by

Jussi K. Vaurio and Dominic Sciaułone*

Reactor Analysis and Safety Division

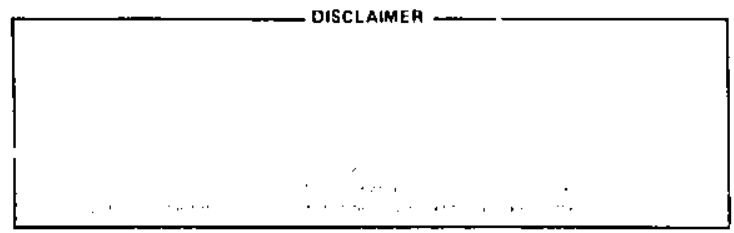

October 1979

*Current address: K1.olls Atomic Power Laboratory, Schenectady, New York 
TABLE OF CONTENTS

$\underline{\text { Page }}$

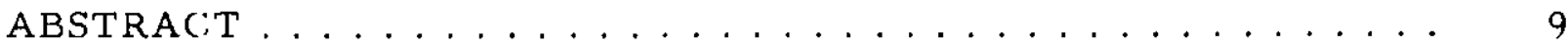

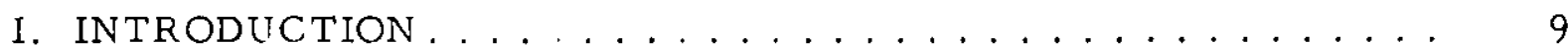

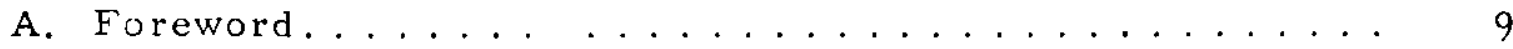

B. Terminology ....................... 10

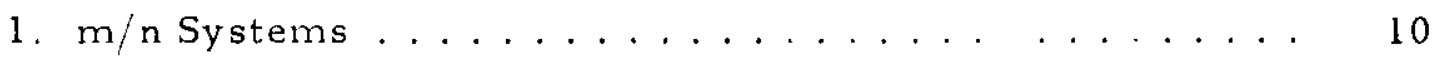

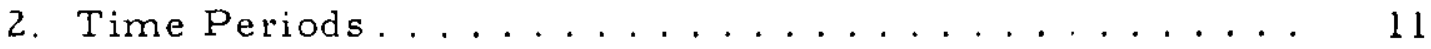

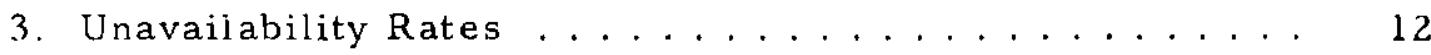

C. Literature Review ......................... 13

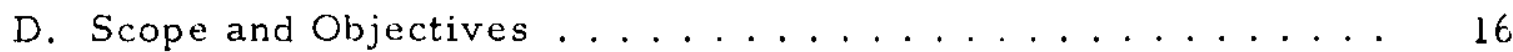

E. Presentation of Material................ 17

II. UNAVAILABILITY EQUATIONS FOR SINGLE-COMPONENT

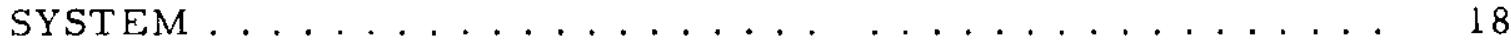

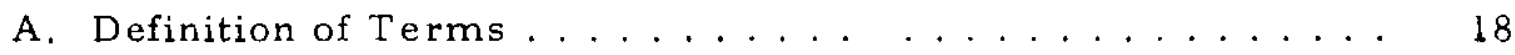

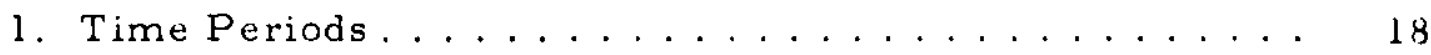

2. Failure Probabilities.................. 18

B. Derivation of Unavailability Equations.............. 20

1. Unavailability during Testing ............. 20

2. Unavailability during Repair .............. 20

3. Unavailability during Operational Standby ......... 20

4. Simplification of Equations ............... 22

C. Average Unavailability ................... 23

D. Optimum Test Interval .................. 23

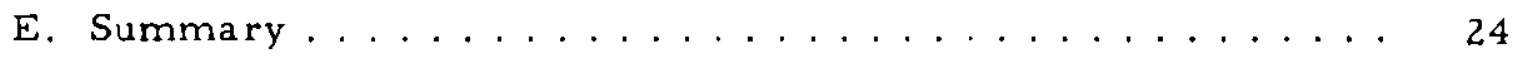

III. SYSTEM UNAVAILABILITY EQUATIONS ............. 25

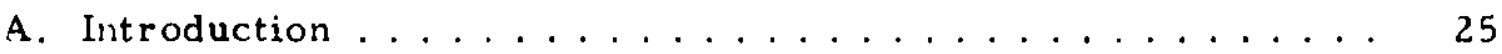

B. Random $\mathrm{Testing}$ Scheme ..................... 25

C. Uniformly Staggered Testing Scheme ............ 25

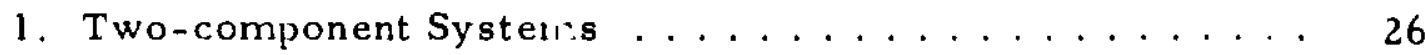

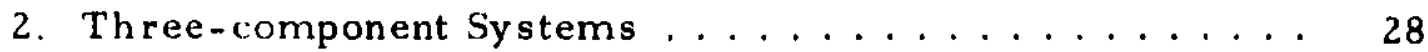

3. Four-component Systems ................... 30 
TABLE OF CONTENTS

$\underline{\text { Page }}$

D. Nearly Simultaneous Testing Scheme ............ 35

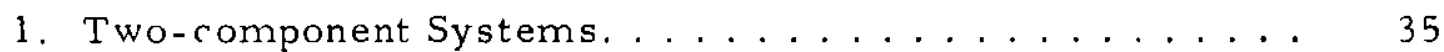

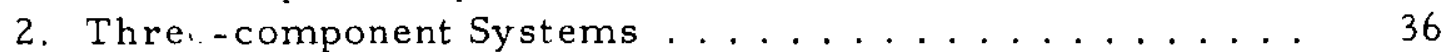

3. Four-component Systems .................... 39

E. Calculation of Optimum Test Interval . . . . . . . . 45

F. Calculation of Fractional Unavailabilities. . . . . . . . 46

G. Summary ............................. 47

IV. COMPARISON OF ICARUS AND FRANTIC . . . . . . . . . 48

A. Introduction .......................... 48

B. Differences between ICARUS and FRANTIC . . . . . . . 48

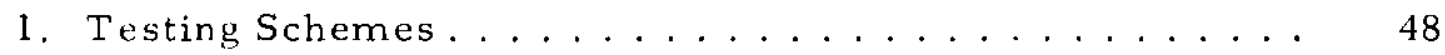

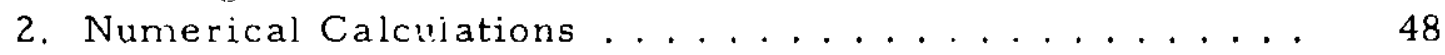

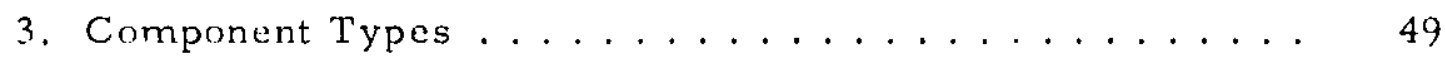

4. Failure Modes .................... 49

C. Comparison of ICARUS and FRANTIC for Simple Systerrs. . . 49

1. Comparison of Uniformly Staggered Testing Scheme . . . 49

2. Comparison of Nearly Simultaneous Testing Scheme.... 49

D. Treatment of Multicomponent Redundancies .......... $5 \mathrm{l}$

1. Test Interval, $\mathrm{T}$ eff $\ldots \ldots \ldots \ldots \ldots$

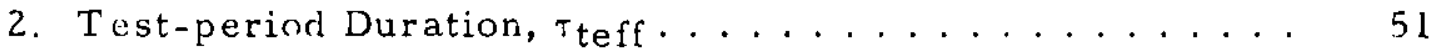

3. Repair-period Duration, ${ }^{\mathrm{r}}$ eff .............. 52

4. Fraction of Downtime during ' esting, qoeff ........ 52

5. Remainder of the Parameters ............. 52

E. ICARUS and FRANTIC Comparisons for Two-valves-per-

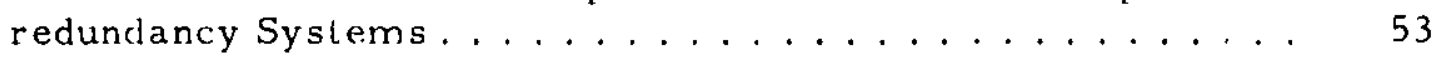

1. Comparison of Uniformly Stagored Testing Scheme .... 53

2. Comparison of Nearly Simultaneous Testing Scheme.... 53

3. Exact Calculations for Average Unavailability of

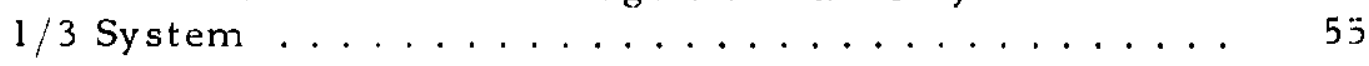

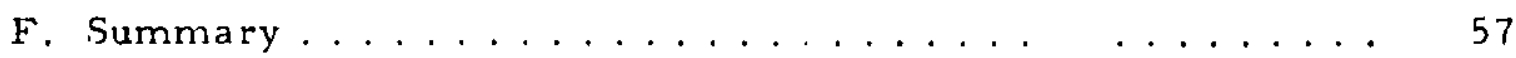

V. ANALYSIS OF AN AUXILIARY FEEDWATER SYSTEM ...... 58

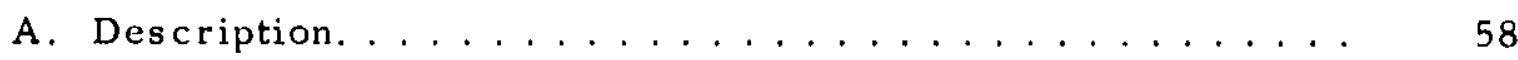


TABLE OF CONTENTS

$\underline{\text { Page }}$

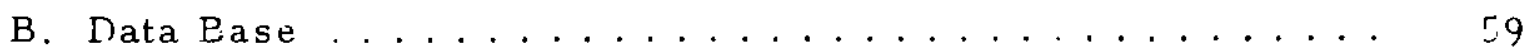

1. Values of $\rho_{u}$ and $\rho_{f} \ldots \ldots \ldots \ldots \ldots \ldots$

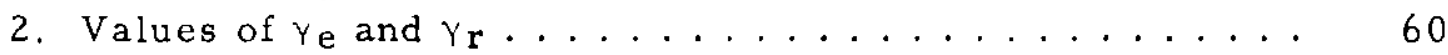

C. Numerical Analysis ..................... 60

1. Case I: Plant Steam Available, Offsite Power Available. . 60

2. Case II: Plant Steam Not Available, Offsite Power

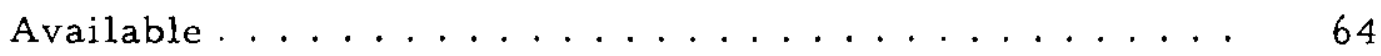

3. Case III: No Steam Available, No Offsite Power

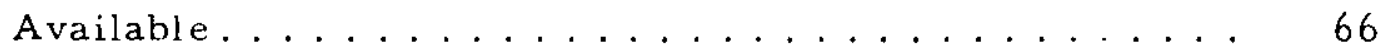

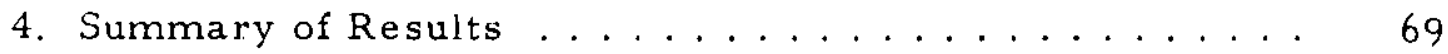

5. Sensitivity of ICARUS Results .............. 71

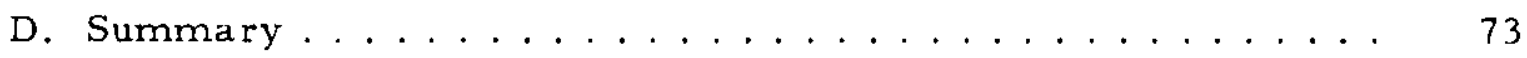

VI. SUMMARY AND CONCLUSIONS .................. 74

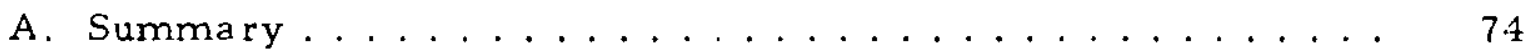

B. Eecommendations for Future Work. . . . . . . . 75

APPENDICES

A. Linearization of $\exp (-\lambda s) \ldots \ldots \ldots \ldots$

B. ICARUS Users' Manual . . . . . . . . . . . . . 77

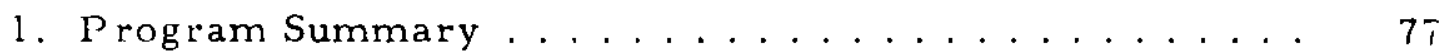

2. Program Abstract .................. 77

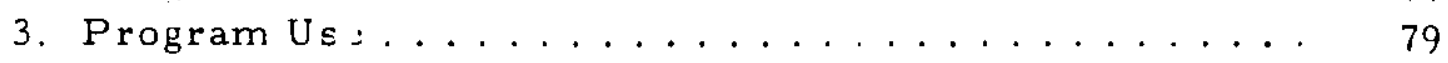

C. Flow Diagrams for ICARUS ................ 82

D. ICARUS Program Listing ................... 86

E. 1 ARUS Input and Output ................. 102

$F$. I)ata Base for $\rho_{u}, \rho_{f}, Y_{e}$, and $\gamma_{r}$ Type Failures ....... 105

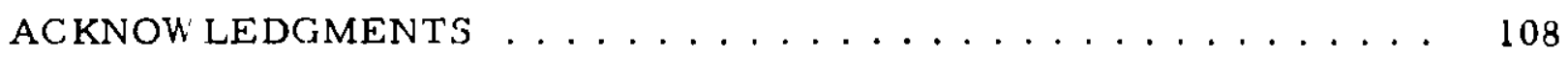

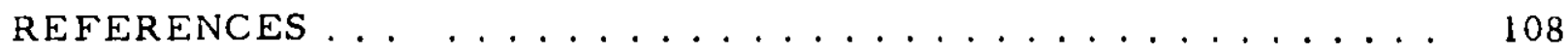




\section{LIST OF FIGURES}

No.

Title

$\underline{\text { Page }}$

1. Single-component Redundancies................ 10

2. Multicomponent Redindancies ................ 10

3. Testing, Repair, and Operational Standby Periods of Component Test Interva! . . . . . . . . . . . . . . . . . . . . 11

4. Uniformly Staggered Testing Scheme ............. 11

5. Simultaneous Testing Scheme ............... 11

6. 1/l System Unavailability Curve from Dressler and Spindler . . $\quad 14$

7. 1/1 System Unavailability Curve from Signorut ......... 15

8. 1/1 System Unavailability Curve from Apostolakis and Bansal. . I5

9. 1/1 System Unavailability Curve from Vesely and Goldberg . . 16

10. Simplified Drawing of Auxiliar Feedwater System . . . . . . . 58

11. System Drawirg for Cases I and II .............66 61

12. Average Unavailability v's Test Interval for Case I . . . . . . 64

13. Average Unavailability vs Test Interval for Case II . . . . . . 66

14. Syster Drawing for Case III . . . . . . . . . . . . 67

15. Average Unavailability vs Test Interval for Case III . . . . . . 69

C.1. Flow Diagram for MAIN Routine ..... . . . . . . . . 82

C.2. Flow Diagram for CALC Subroutine ........... 84

C.3. Flow Diagram for ITERAT Subroutine ............ 85 


\section{LIST OF TABLES}

No.

Title

$\underline{\text { Page }}$

I. Variable Symbols and Definitions ............. 19

II. Unavajlability Equations during Testing. Repair, and

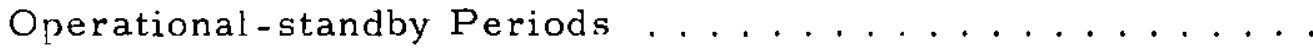

III. Average Unavailability Equations for $\mathrm{m} / \mathrm{n}$ Systems with Random Testing Scheme .................... 25

IV. Errurs in $(T / 2)^{n}-\sigma^{n}=(T / 2)^{n}$ Approximation ........ 27

V. Errors in $\left(\mathrm{I}^{\prime} / 3\right)^{\mathrm{n}} \quad \theta^{\mathrm{n}}=(\mathrm{T} / 3)^{\mathrm{n}}$ Approximation $\ldots \ldots . \ldots 27$

VI. Errors in $(\mathrm{T} / 4)^{\mathrm{n}}-\mathrm{O}^{\mathrm{n}}=(\mathrm{T} / 4)^{\mathrm{n}}$ Approximation ........ 27

VI. Errors in $T^{n}-\left(\theta+T_{t}\right)^{n}=T^{n}$ Aproximation ......... 36

VIII. Errors in $T^{n}-\left(\beta+2 \tau_{t}\right)^{n}=T^{n}$ Approximation ........ 37

IX. Errors in $\mathrm{T}^{\mathrm{n}}-\left(\theta+3 \mathrm{~T}_{\mathrm{t}}\right)^{\mathrm{n}}=\mathrm{T}^{\mathrm{n}}$ Approximation ........ $4 \mathrm{l}$

$\mathrm{X}$. Equation Numbers for System Unavailability . . . . . . . . . 45

XI. Input Data for Single-valve Redundancy. . . . . . . . . . 50

XII. Comparison of ICARUS and ERANTIC Results for Uniformly Staggered Testing ................... 50

XIII. Comparison of ICARUS and FRANTIC Results for Nearly Simultaneous Testing ................... 51

XIV. ICARUS and FRANTIC Input Values for Multicomponent Redurdancies ......................... 53

XV. Comparison of ICARUS and FRANTIC Results for Two Valves per Redundancy and Uniformly Staggered Testing . . . . . . 54

XVI. Comparison of ICARUS and FRANTIC Results for Tivo Valves per Redundarcy and Nearly Simultaneous Testing. . . . . . . 54

XVII. Parameter Values for Test System . . . . . . . . . . 56

XVIII. ICARUS Results for Case I. . . . . . . . . . . 63

XIX. ICARUS Results fur Case II .................. 65

XX. ICARUS Results for case III . . . . . . . . . . . . 68

XXI. Summary of Data from All Cases . . . . . . . . . . . 70

XXII. Results of Sensitivity Studies . . . . . . . . . . . . 72

A.1. $\lambda s$ vs $1-\exp (-\lambda s) \ldots \ldots \ldots \ldots$ 



\title{
UNAVAILABILITY MODELING AND ANAI,YSIS OF REDUNDANT SAFETY SYSTEMS
}

by

Jissi K. Vaurio and Dominic Sciaudone

\begin{abstract}
Analytical expressions have been devaloped to estimate the average unavailability of an $m$-out-of $-n(m / n, 1 \leq m \leq n \leq 4)$ standby safety system of a nuclear power plant. The expressions take into account contributions made by testing, repair, equipment failure, human error, and different testing schemes. A computer code, ICARUS, has beeis written to incorporate these analytical equaticns. The code is capable of calculating the average unavilability, optimum test interval, and relative contributions of testing, repair, and randron failures for any of three testing schemes.

After verification of the methodology and coding in ICAR! S, a typical auxiliary feedwater system of a nuclear power plant was analyzed. Tile results show that the failure modes as sociated with testing and true demands contribute considerably to the unavailability and that Diesel generators are the most critical components contributing to the overall unavailability of the system.
\end{abstract}

\section{INTRODUC' 1 ION}

4. Forevord

Tne devclopment of nuclear power as a viable energy source has put added imphasis on making reactor systems as safe as possible. Reactor designers bear the responsibility of designing systems in which accidents are rare and of developing special systems to mitigate the consequences of an accident if one should occur. As a result, redundant standby safety systems a re built into nuclear power plants. These systems do not operate under normal conditions, but should be reidy to respond if some emergency situation develops. The emiergency core cooling system (ECCS) and the auxiliary fer:dwater system (AFWS) are two examples of this type of standby safety system. These systems are periodically tested to ensure proinpt availability upon dimand. 
Due to the nature of mechanical components and the human inter actions with them, there is a finite probability that the siandby system may fail to function once it has been called upon. The factors contributing to this unavailability may range from the random failure of a component in the system, to simply taking the componert out of service to test its operability. A method capable of estimating the unavailability of a system as a function of various types of failure modes would greatly aid in determining which factors contribute most to the unavailability and may lead to modifications to the system or maintenance schedules that would rrduce the probability of failing to siart upon demand.

B. Terminology

Before discussing some of the previous work done on this subject, we will present some basic background and terminology.

\section{1. $1 \mathrm{n} / \mathrm{n}$ Systems}

-1 systcm is designated as being m-out-of-n $(\mathrm{rr} / \mathrm{n})$ when it is made up of $n$ reduntancies, $m$ of which must be available for the system to be available. Clearly, $m$ inust be less than or cqual to $n$. For example, an emergency power system may consist of three separate devices, any one of which c. 1 supply the remand. This system is designated as being a $1 / 3$ system.

Each redundancy and system may be composed of single components in parallel (Fig. 1) or a series of components in parallel (Fig. 2). For simplicity, further references will be to single-rather than multicomponent redundancies ivher" cath redundancy will be referred to as a component.

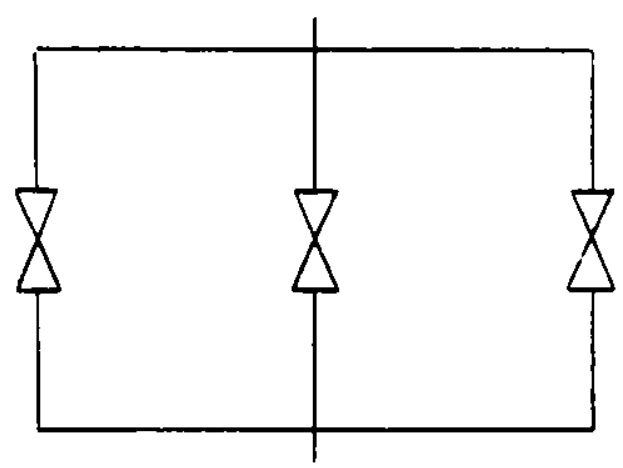

Fig. 1, ingle-component Redundancies

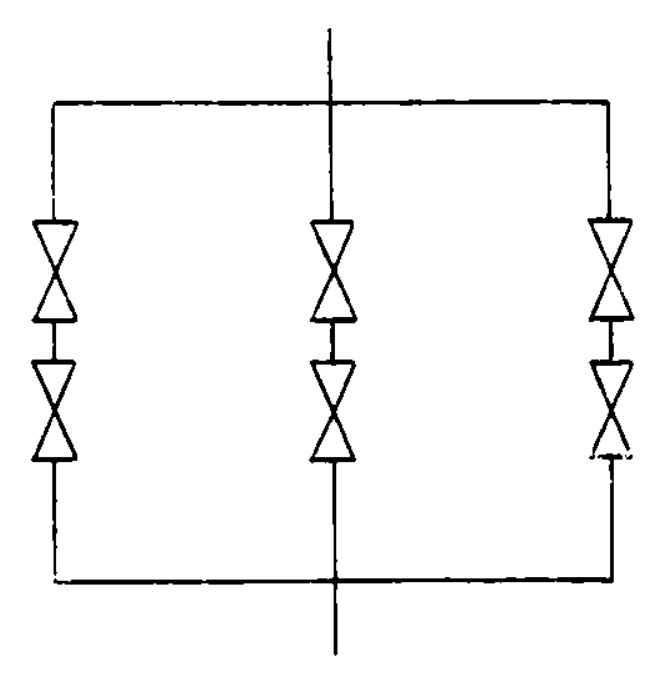

Fig. 2. Multicomponent Redundanc ies 
Each component in the system is tested periodically, and the following definitions are used: test interval, test period, repair period, and operational standby period.

\section{Time Periods}

The test perlod is the time when the component is checked to see if it is operational. If necessary, the component then goes into a repair period. Here, all repairs are done and the component is retested to make sure the repair was successful. Finally, the tested and repaired component goes on operational standby until the next test period. A test interval is the time between the start of successive test periods. These periods are shown schematically in Fig. 3.

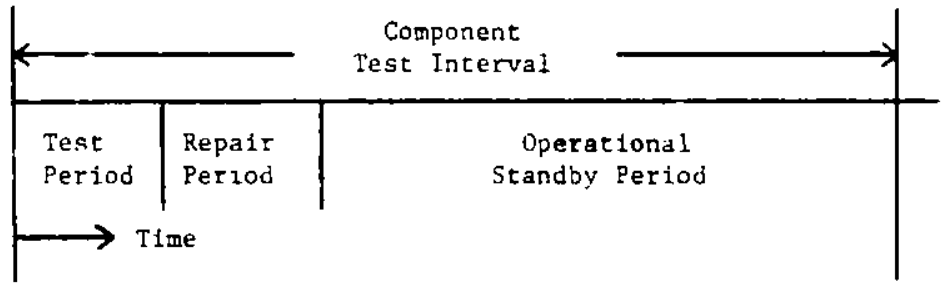

Fig. 3. Tesung, Repair and Operational standisy Periods of cimponent Test interval
A test interval can be identified for an individual component within a system or for the system itself. In either case, the test interval will be determinerl by the testing scheme, which may be uniformly staggered, simultaneous, or random. In the uniformly staggered testing scheme, the testing of the components is performed at equally spaced times throughout the test interval as indicated in Fig. 4 . In the simultaneous testing scheme, all $\mathrm{n}$ components are tested at the beginning of the test interval, as shown in Fig. 5. No two components a re voluntarjiy taken out of service exactly simultaneously, but one after another. Finally, in the random testing schtme, each component is tested at randomly shifted times during the test interval.

\section{Fig. 4}

Uniformly staggered Testing scheme (Threc-component system, 720-h test interval)

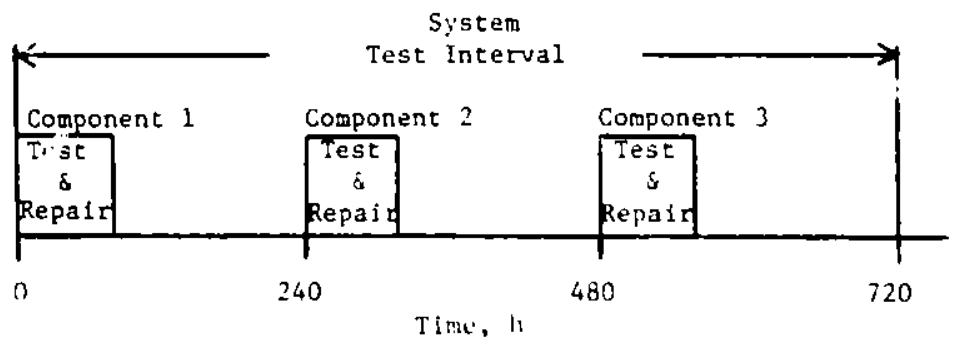

Fig. 5

simultaneous Testing Scheme (Threc-component system. 72u-h test interval) 


\section{Unavailability Rates}

If a component has only random mechanical failures, then its availability or reliability is simply a function of its random failure rate, $\lambda$. If $R(s)$ is the arailability at some time $s$, then the availability at any time $s: d s$ can be written as

$$
R(s+d s)=R(s)(1-\lambda d s)
$$

where $\lambda \mathrm{ds}$ is the probability of the component failing during time ds.

The availability at any time $s$ is found by rearranging Eq. I and by the calculus to yield

$$
\mathrm{R}(\mathrm{s})=\exp \left(-\int_{0}^{\mathrm{s}} \lambda \mathrm{ds}\right)
$$

where it is assumed that $R(0)$ is unity.

If $?$ is constant during the time interval of interest,

$$
R(s)=\exp (-\lambda s)
$$

Another way to use this result is to note that, if the availability falls off exponentially, then the failure rate is a constant and can be interpreted as being equal to the reciprocal of the mean time to component failure. ${ }^{1}$

The unavailability of the component is defined as the complement of the availability and is given the symbol u(s). Thus, for a constant failur $\epsilon$ rate,

$$
u(s)=1-\exp (-\lambda s)
$$

The unavailability of a component at the end of a test interval due to random failures unly during the test interval $T$, is given by

$$
u(T)=1-\exp (-\lambda T)
$$

However, at the end of an arbitrary test interval, we would expect the unavailability to be at some different value than this, since the component rnay have been taken out of service during testivis or underwent some repair curing the test interval. An asymptctic state is defined when the value of $u(T)$ no linger changes so that the unavailability at any. unavailability at that same $t$ me in any subsequent test intervai. This idealizaion is a sensible approximation, especially after two or three test intervals, when the initial system faults have been worked out. 
The average unavailability $\overline{\mathrm{u}}$ is defined as

$$
\bar{u}(\Gamma)=\frac{\int_{0}^{T} u(s) d s}{\int_{0}^{T} d s} .
$$

In the asymptotic state, $\bar{u}$ is a function of only the test interval, $T$, not of the absolute time from the beginning of the plant operation. There exists an optimum value for the test interval such that the lowest average asymptotic unavailability for the system will be achieved. This value, $T_{0}$, is defined by

$$
\frac{\mathrm{d} \overline{\mathrm{u}}}{\mathrm{dT}}=0
$$

If a system undergoes only random failures, then the average unavailability can be determined by Eqs. 3 and 5 to be

$$
\bar{u}(T)=1-1 / \lambda T+(1 / \lambda T) \exp (-\lambda T)
$$

Using Eq. 6, we find the optimum test interval $T_{0}$ to be

$$
\mathrm{T}_{0}=0
$$

This result means that, as long as the component is continually tested, the system is always available. Although this is not realistic since, for example, downtime during testing has been neglected, Eqs. 5 and 6 can be applied to more rigorously derived unavailability equations and more meaningful values of the average unavailability and optimum test intervals can be determined. These topics are covered in Chapter II of this report.

C. Lite rature Review

Several researchers have addressed the subject of unavailability analysis and optimum testing intervals. Dressler and Spindle ${ }^{2}$ have analytically calculated the time-dependent and average unavailabilities for all periodically tested safety systems with $\mathrm{m} / \mathrm{n}(\mathrm{l} \leq \mathrm{m} \leq \mathrm{n} \leq 4)$ redundancy for both staggered and simultaneous testing schemes. 'The methodology assumes constant failuı rate, repair time, and test intervals, along with a test-period duration of zero. Testing is assumed not to take the component "off-line," thus allowing no errors during testing.

The results for a $1 / 1$ system give rise to a sawtooth-shaped curve shown in Fig. 6. Since the failure of the component is due only to random causes, the optimum test interval is zero. Although many realistic failure 
modes are lidissing in this model, it contains perhaps the most comprehensive analytical equations currently available for the unavailability of $\mathrm{m} / \mathrm{n}$ standby systems.

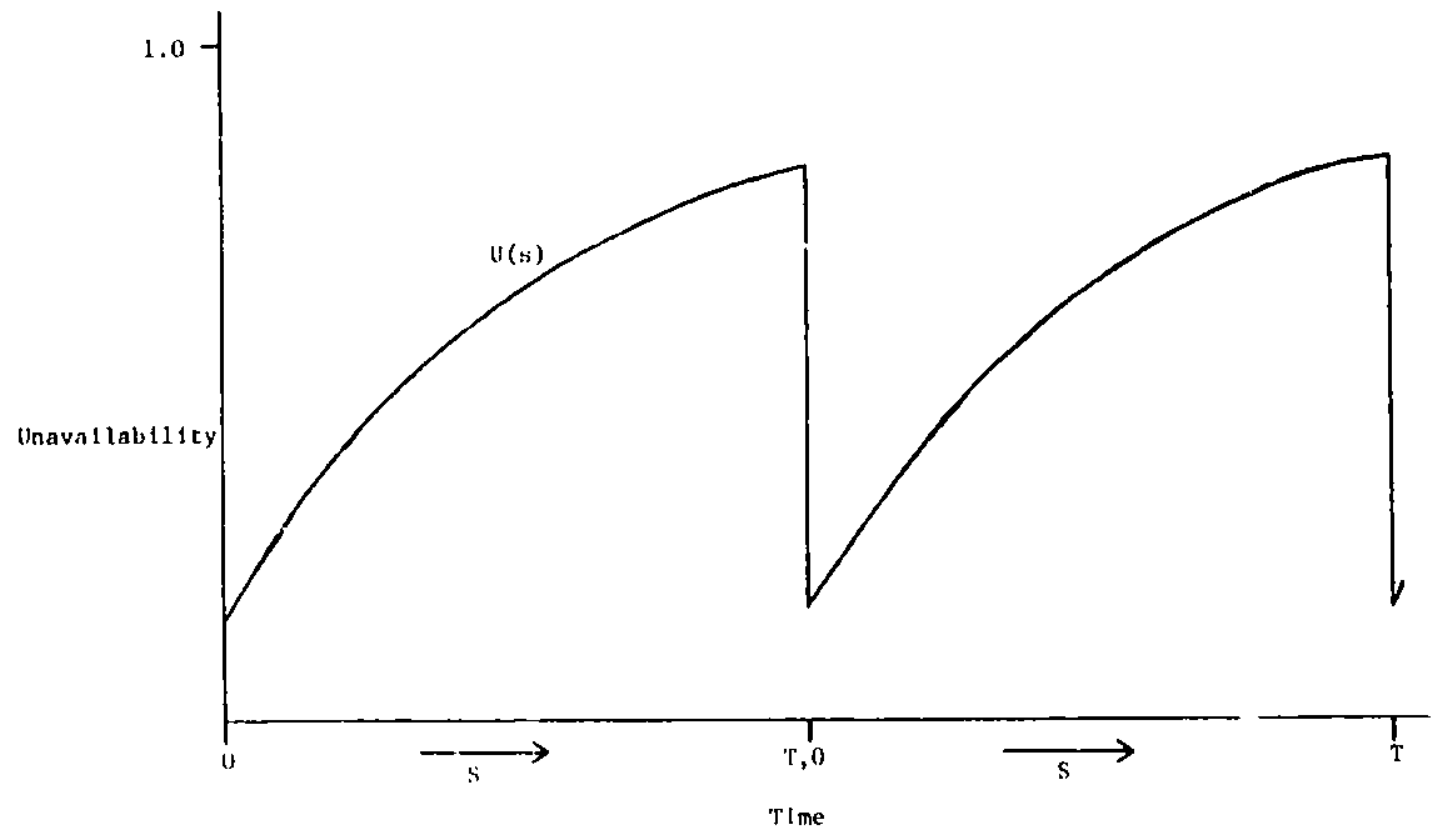

Fin. 1. 1/1 system Unavailability Curve from Dessler and spindler ${ }^{2}$

Signoret ${ }^{3}$ attempts to analyze the contributions made to component failure not only from random failures, but from other sources as well. As in the previous reference, the failure rate and test interval a re assumed to be conrtant and the test duration is zero. The repair period, however, is pres.. $d$ in the form of a constant repair rate or mean time to repair. Also included is the probability of failing to start due to a test-caused failure and the probabilizy of failing to stari due to a true demand. These results give rise to the curve in Fig. 7.

The discontinuity of the curve at $s=0, T, 2 \mathrm{~T}$, etc., is due to the probability that a test fails the component. The curve then drops sit exporientially as a result of the constant repair rate before incrcasing again due to the constant failure rate. Analytical expressions are not derived for the other $\mathrm{m} / \mathrm{n}(\mathrm{m}, \mathrm{n}>1)$ systems. Instead, $1 / 1$ curves such as is found in Fig. 7 for various components arc multiplied or added together to get numerical results for multicomponent systems.

A further step toward a more realistic assessment of safety-system unavailability has been taken by Apostolakis and Bansal. ${ }^{4}$ An attempt is made to recognize the contributions of human errors and common-cause errors to system unavail bility where a finite probability is given for leaving a tested component in a lailed state. The probability of failure due to a real demand is also taken into account. 


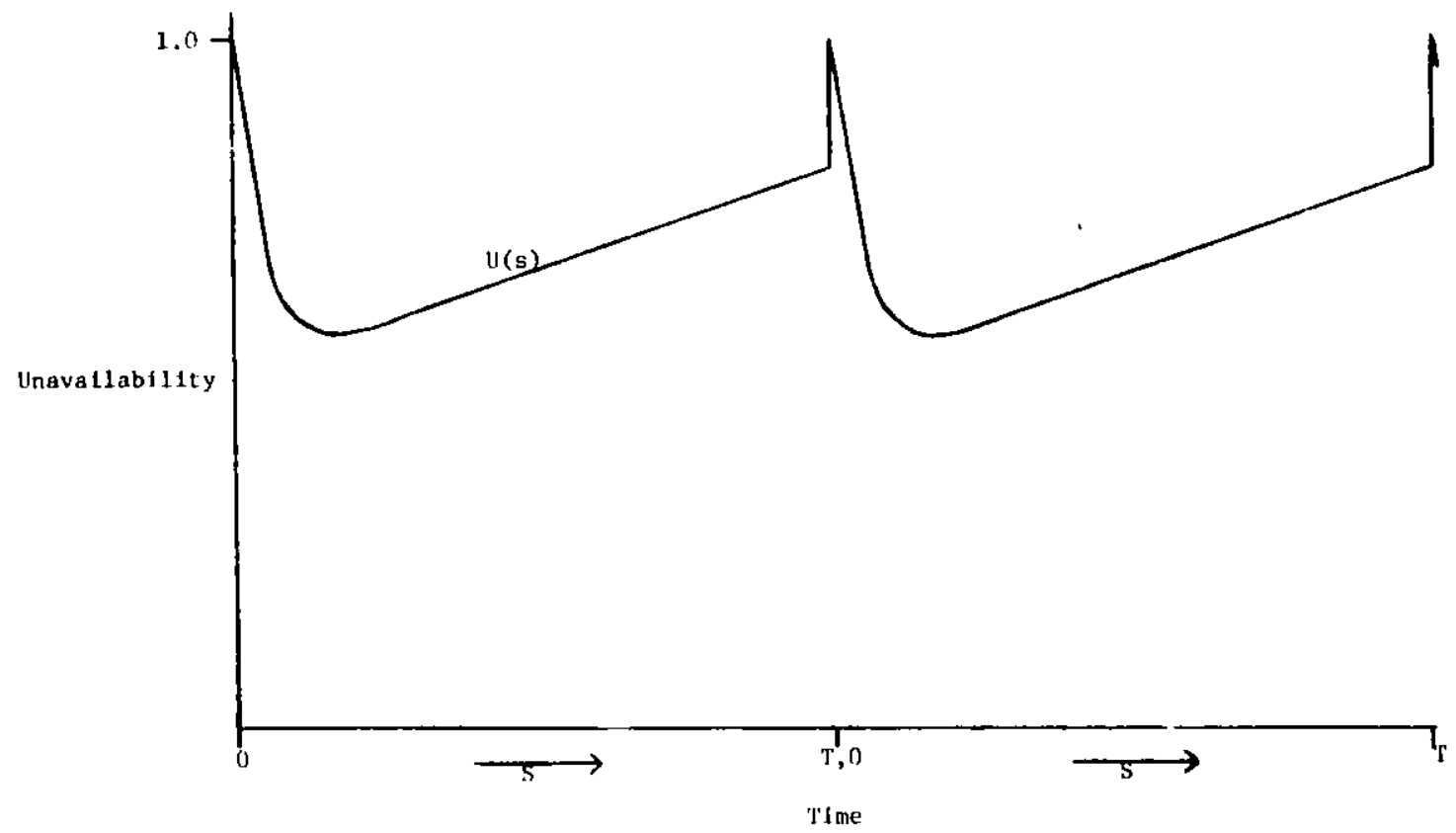

Fig. 7. 1/1 System Unavailability Curve from Signoret ${ }^{3}$

Other assumptions made by Apostolakis and Bansal are the use of constant failure rates, constant test interval, and constant test duration. Furthermore, it is assumed that the compon'nt is down throughout the test period and that this period includes all the repair time seded to bring the component on line. The unavailability as a function of time for this rethod is found in Fig. 8. Note that the component is completely unavailable $[u(s)=$ 1.0] throughout the test $\left(0<s \leq \tau_{t}\right)$. Another feature of this curve is the linearization of the random-failure contribution to the operationalstandby-phase uncvailability. This assumption is discussed further in Appendix A.

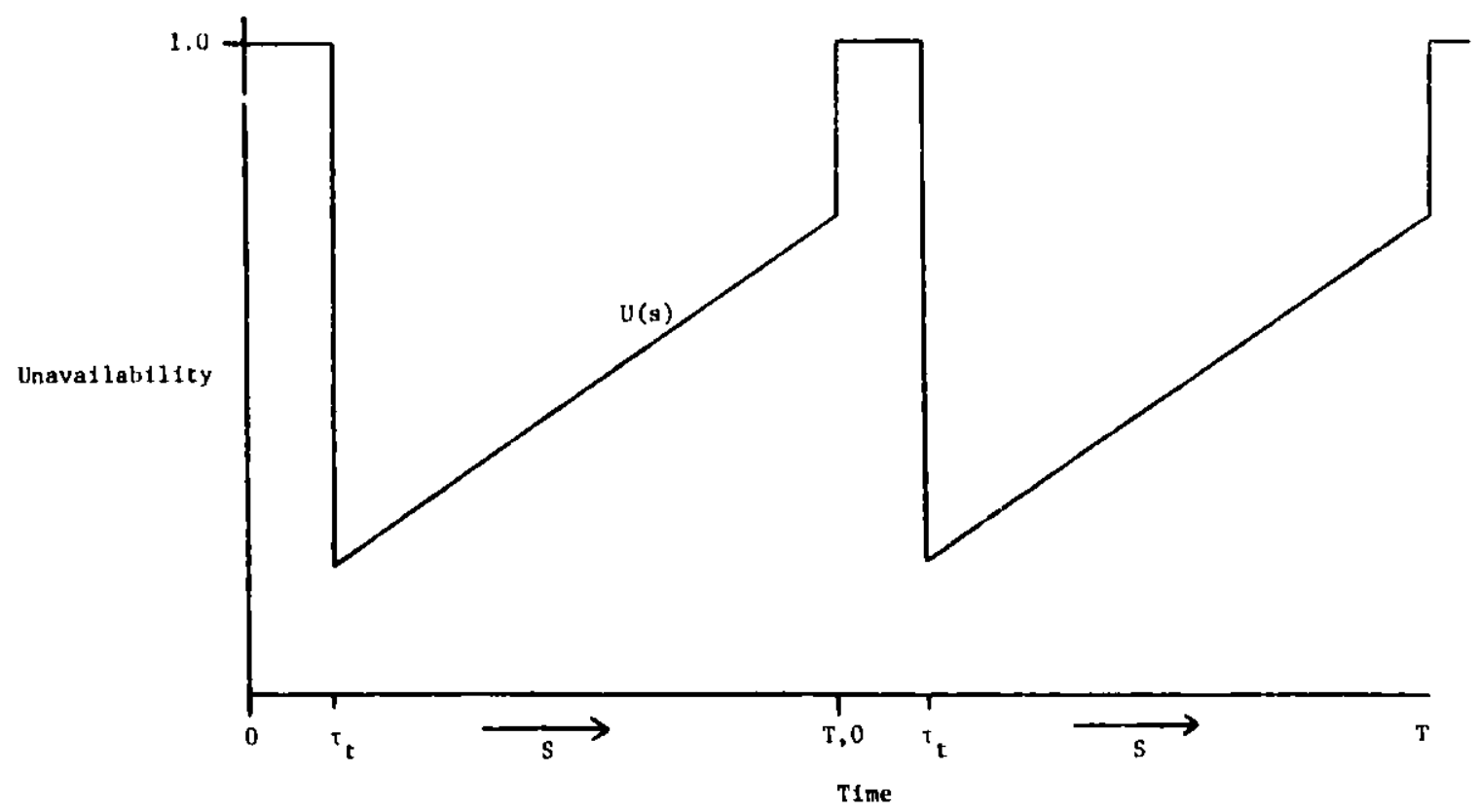

Fig. 8. 1/1 System Unavailability Curve from Apostolakis and Bansal ${ }^{4}$ 
Vesely and Goldberg ${ }^{i, 6}$ have dereloped the FRANTIC computer code, which calculates time-dependent and average unavailabilities due to the effects of test downtime, repair time, test-caused failures, and test staggering. The failure rate, test interval, test time, and repair time arc constant, and all detectable failures are assumed to be detected and repaired. A typical unavailability curve for the FRANTIC code methodology is shown in Fig. 9. Note that the unavailability is constant during testing $\left(0<s \leq \tau_{t}\right)$ and repair $\left(\tau_{t}<s \leq \tau_{t}+\tau_{1}\right)$ and that the linearization for the rardom-failure contribution is used. The FRANTIC code is cliscussed furthor in Chapter IV.

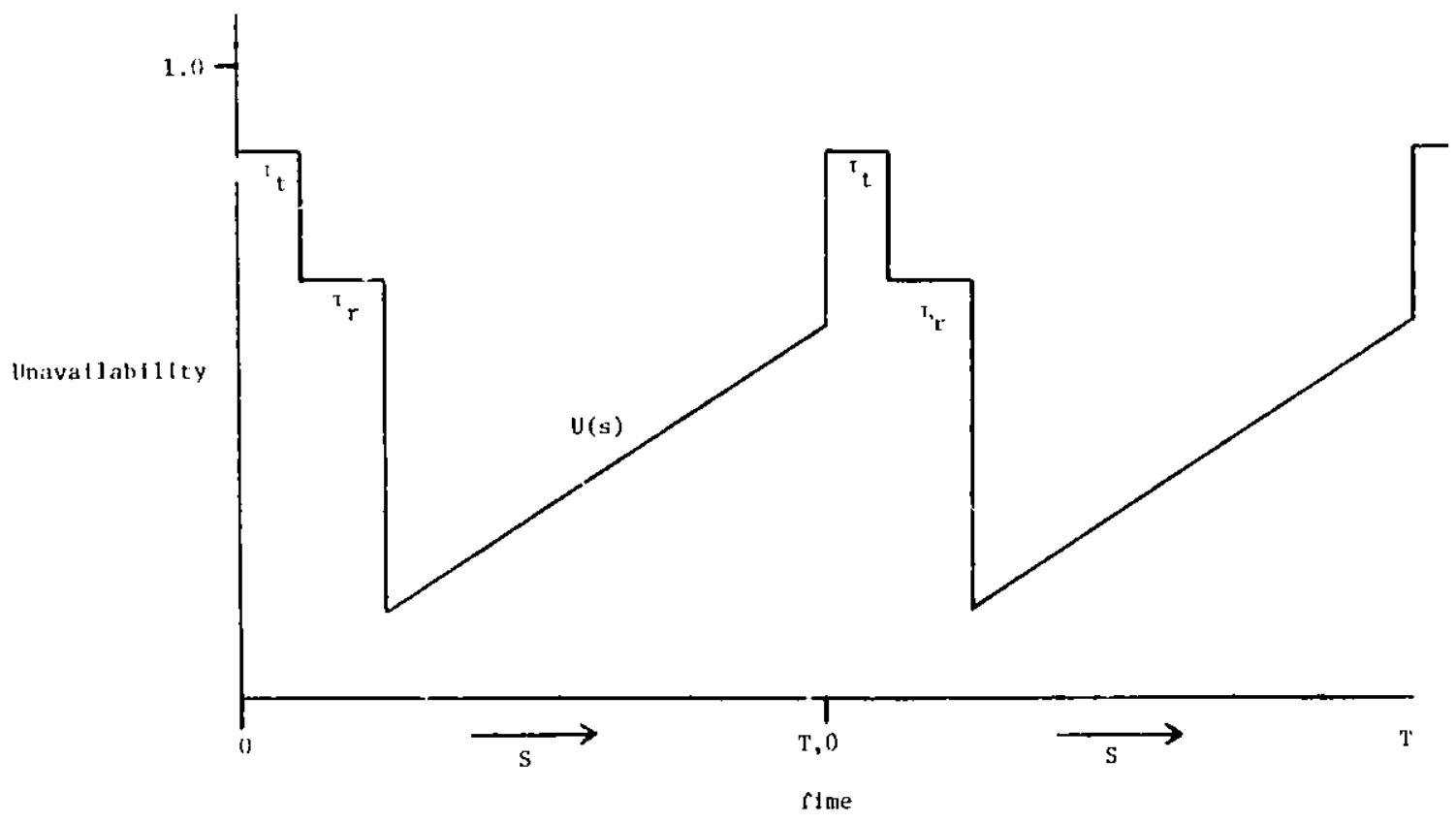

Fịn. 9. 1'1 system Unavailability Curve from Vesely and cioldberg ${ }^{5}$

A comprchensive unavailability model has been developed by Vaurio ${ }^{7}$ for a single standby component and for a series system consisting of several components. Reference 7 can be viewed as a theoretical framework and basis for many assumptions made in this work.

D. Scope and Objectives

The scope of the present work is to identify as many types of failure modes as possible and to include them in the development of analytical expressions for estimating the unavailability of redundant safety systems. (The word "type" is used in this context to distinguish between human, random, and mechanical failures.) To achieve this, the basic equations from FRANTIC 6 are modified and new variables incorporated to represent new types if failures. Average unavailabilities are then calculated for all $\mathrm{m} / \mathrm{n}$ systems $(\mathrm{l} \leq \mathrm{m} \leq \mathrm{n} \leq 4)$ for uniformly staggered, nearly simultaneous, and random-testing schemes. In addition to this, the optimum test interval and fractional una vailabilities due to testing, repair, and random failure are evaluated. 
A computer code, ICARU'S, has been written to inccrporate the new equations. Since the exact integrals and derivatives indicated in Eqs. 5 and 6 have been analytically programmed, no time-consuming numerical methods are used in arriving at the average unavailabilities and optimum test intervals. Consequently, the runring time is short, and coupling with the probabilistic response-surface code $\mathrm{PROSA}^{8}$ to do sensitivity analysis of the input data is facilitated. Several sets of simple systems have been analyzed with ICARUS to test the efficacy of the new equations and programming.

A final objective of this work is to apply the ICARUS corle to an actual standby iafety system. Using data collected from various sources, we analyzed an auxiliary feedwater system of a pressurized water reactor (PWR).

E. Presentation of Material

Chapter II describes the basic equations for treating a single-component (1/1) system. Each parameter is defined, and the unavailability equations for testing, repair, and operational standby periods are developed.

The system average unavailability equations are derivod in Chapter III for $\mathrm{m} / \mathrm{n}$ systems where $1 \leq \mathrm{m} \leq \mathrm{n} \leq 4$. Included are the system unavailability equations for the random, uniformly staggered, and nearly simultaneous testing schemes that have been programmed into the ICARUS computer code.

Chapter IV compares the ICARUS and the FRANTIC computer codes. A short description of FRANTIC is followed by a comparison of results between the two codes for single- and multicor, tponent redundancy systems. Details of the ICARUS computer code including flow charts, listing, and a users' manual are given in the appendices.

An auxiliary feedwater system is described and analyzed using the ICARUS code in Chapter V. An analysis is performed to determine the sensitivity of selected output variables to the it data.

Finally, in Chapter VI, the conclusions drawn from this research are presented alcing with recommendations for future work. 


\section{UNAVAILABILITY EQUATIONS FOR SINGLE-COMPONENT SYSTEM}

In this chapter, the terms used in the development of the unavailability equations are defined and discussed. Also, the unavailability equations for the test, repair, and operational-standby periods are developed leading to the average unavailability and optimum test interval of a 1/l system.

A. Definition of Terms

Although some of the methods and ideas developed by the authors of previous works were the same, different symbols were used in many cases to represent the same concept. The most commonly used symbols will be used in this work.

\section{Time Periods}

The test interval, $T$, is defined as the time between the starts of two consecutive tests for any one component. The variable $s$ is the time since the beginning of the interval. The intw val, therefore, starts at $s=0$ and ends at $s:=T$. The test interval is typically one month $(720 \mathrm{~h})$ long.

The beginning of a test interval is marked by a componont test. The length of this period, $\tau_{i}$, depends on the component undergoing lesting. Immediately after the test, a repair period is begun and lasts for a period $\tau_{r}$. Again the length of $T_{r}$ is dictated by the component. The sum of the test and repair times is given the symbol $\theta$. At time $s=\theta$, the component is ready to go into operational standby for the remainder of the test interval.

\section{Failure Probabilitics}

During the test period, a component may be taken "off-line" for a certain amount of time to perform the test. The variable, $q_{l}$, is defined as the fraction of the test period that the component is unavailable to answer a real demand.

There is a finite probability that when the safety system is tested, the very act of operating the component may cause it to fail. This can occur in two ways in the testing period. With a probability of $\gamma_{e}$ (per test), a failure is caused that is detected and the component is repaired and ready to go into operational standby at $s=\rho$. With a probability of $\rho_{u}$ (per test), a failure is caused that is not repaired after the test. This nonrepair could be due to human negligence or sume type of hidden failure not detected by testing.

The component sreaks down on the initiation of a real or true demand with a probability of $\gamma_{r}$ (per true demand). The distinction between $\gamma_{e}$ and $\gamma_{r}$ is the initiating event. Human errors may play a larger role in $\gamma_{e}$ and $\rho_{u}$ than in $\gamma_{r}$. 
This work assumes that true demands are rare events compared to the testing frequency. With this assumption, the unavailability (= the protability that the component does not perform its function if needed at time s) can be written as ${ }^{7}$

$$
u(s)=\gamma_{r}+\left(1-\gamma_{r}\right) u\left(s \mid \gamma_{r}=0\right),
$$

where $u\left(s \mid \gamma_{r}=0\right)$ contains contributiuns from all failures other than those caused by true demands.

A comporent may go into a test in a failed state due to the failurerate contribution $\lambda$, or any of the test-related failures. This probability is designated $\hat{\mathrm{u}}$. It may, however, not be detected as bein; failed, or, if detected, not properly repaired. This may te due to human testing error, repair error, or some kind of detection error. This is defined as occurri. $g$ with a probability of $\rho_{f}$ (per test).

The ternis and their meanings relative to this work are summarized in Table $I$.

TABLE I. Variable Symbols and Definitions

\begin{tabular}{|c|c|}
\hline $\mathrm{T}$ & Test interval \\
\hline $\mathbf{s}$ & Time since beginning of test inte ryal \\
\hline$\tau_{\mathrm{t}}$ & Test-period duration \\
\hline$\tau_{r}$ & Repair-period duration \\
\hline$\theta$ & Sum of $\tau_{t}$ and $\tau_{r}$ \\
\hline $\mathrm{q}_{0}$ & Fraction of $\tau_{t}$ that component is down \\
\hline$\gamma_{\mathrm{e}}$ & $\begin{array}{l}\text { Probability of failure due to a test demand that } \\
\text { is repaired }\end{array}$ \\
\hline $\mathrm{Pu}_{\mathrm{u}}$ & $\begin{array}{l}\text { Probability of failure due to a test demand that } \\
\text { is not repaired }\end{array}$ \\
\hline$\gamma_{r}$ & Probability of failure due to a true demand \\
\hline$\rho_{f}$ & $\begin{array}{l}\text { Probability that a failed component is not detected } \\
\text { by a test or not repaired }\end{array}$ \\
\hline$\lambda$ & Failure rate of component \\
\hline$\hat{\mathbf{u}}$ & Probability that a component enters the lest failed \\
\hline 8 & $\rho_{\mathrm{f}}-\rho_{\mathrm{u}}$ \\
\hline $\bar{\alpha}$ & $\begin{array}{l}1-\alpha, \text { where } \alpha \text { is any of the previously defined } \\
\text { probabilities }\end{array}$ \\
\hline
\end{tabular}




\section{B. Derivation of Unavailability Equations}

1. Unavailability during Testing

A component may be unavailable in its test period because it came into the test failed, it failed during the test, or it may be down a fraction of the test time to perform the test. These events are mutually exclusive, rud by using the definitions in Table $I$, the unavailability during testing, $u_{\mathrm{T}}$, can be written as

$$
\begin{aligned}
0<s \leq \tau_{t}, u_{T}(s)= & q_{0}+\bar{q}_{0} \gamma_{e}+\bar{q}_{0} \bar{\gamma}_{e} \bar{\gamma}_{r}+\bar{q}_{0} \bar{\gamma}_{e} \bar{\gamma}_{r} \rho_{u}+\bar{q}_{0} \bar{\gamma}_{e} \bar{\gamma}_{r} \bar{\rho}_{u} \hat{u} \\
& +\bar{q}_{0} \bar{\gamma}_{e} \bar{\gamma}_{r} \bar{\rho}_{u} \overline{\hat{u}} \lambda s,
\end{aligned}
$$

where

$\lambda s=$ linearized probability of random component failure. (See Appendix A for further discussions.)

If $\gamma_{\mathrm{e}}, \gamma_{\mathrm{r}}$, and $f_{\mathrm{u}}$ are small $(\ll 1), * \mathrm{Eq}$. 7 can be written as

$$
0<s \leq \tau_{t}, u_{T}(s)=q_{0}+\left(1-q_{0}\right)\left[\gamma_{e}+\gamma_{r}+\rho_{u}+\hat{u}+\left(1-\hat{u} i_{i s}\right] .\right.
$$

2. Unavailability during Repair

$\therefore$ component in its repair period may be unavailable due to a failure occurring before or during the repair period. Again these are mutually exclusive, and the unavailability can be written as

$$
\tau_{t}<s \leq \theta, u_{R}(s)=\gamma_{e}+\bar{\gamma}_{e} \gamma_{r}+\bar{\gamma}_{e} \bar{\gamma}_{r} \rho_{u}+\bar{\gamma}_{e} \bar{\gamma}_{r} \cdot \vec{\rho}_{u} \hat{u}+\bar{\gamma}_{e} \bar{\gamma}_{r} \bar{\rho}_{u} \overline{\hat{u}} s .
$$

Again note that, if $\gamma_{e}, \gamma_{r}$, and $\rho_{u}$ are small $(\ll 1)$, Eq. 9 becomes

$$
\tau_{t}<s \leq \theta, u_{R}(s)=\gamma_{\epsilon}+\gamma_{r}+\rho_{u}+\hat{u}+(1-\hat{u}) \lambda s .
$$

\section{Unavailability during Operational Standby}

In this period, a component may be failed because of a previous event, cr may fail due tu a real demand or random failure. The unavailability during this time can be written as

$$
\theta<s \leq T, u(s)=\gamma_{r}+\bar{\gamma}_{r}\left(\hat{u}_{\rho_{f}}+\overline{\hat{u}}_{\rho_{u}}\right)+\bar{\gamma}_{r}\left[1-\left(\hat{u}_{\rho_{f}}+\overline{\hat{u}}_{\rho_{u}}\right)\right] \lambda s .
$$

The term $\hat{u}_{\rho f}+\overline{\hat{u}}_{\rho_{u}}$ evolves from the fact that the probability of a failure not being detected prior to testing can only be valid if the component is failed before the test $(\hat{\mathrm{u}})$, and if it was not failed ( $\overline{\hat{u}})$, the pu probability is valid.

${ }^{*} \gamma_{e} \gamma_{\mathrm{r}}$, and $\rho_{\mathrm{u}}$ are usually on the order of $10^{-3}$. 
Neglecting second-and higher-order terms gives

$$
\theta<s \leq T, u(s)=\gamma_{r}+\rho_{u}+\left(\rho_{f}-\rho_{u}\right) \hat{u}+\lambda s .
$$

Using Eq. 12, we can develop an expression for $\hat{u}$. It was previ.. ously said that $\hat{u}$ was the probability of entering the test period in a failed state. This would correspond to $u(T)$, given that there were no true demand failures $\left(\gamma_{r}=0\right)$. This line of thought is used since true demands are few (i.e., rare events), and if a failure during a true demand should occur, steps would be taken to repair the component immediately. Thus,

$$
\hat{\mathrm{u}}=\mathrm{u}\left(\mathrm{T} \mid \gamma_{\mathrm{r}}=0\right) \text {, or } \hat{\mathrm{u}}=\rho_{\mathrm{u}}+\lambda \mathrm{T}+\hat{\mathrm{u}}\left(\rho_{\mathrm{f}}-\rho_{\mathrm{u}}\right)
$$

and

$$
\dot{u}=\frac{\rho_{u}+\lambda T}{l-\delta},
$$

where

$$
\delta=\rho_{\mathrm{f}}-\rho_{\mathrm{u}} .
$$

Equation 13 can be inserted into Eq. 8, giving

$$
\begin{aligned}
0<s \leq \tau_{\mathrm{t}}, u_{\mathrm{T}}(\mathrm{s})= & \mathrm{q}_{0}+\left(1-\mathrm{q}_{0}\right)\left[\gamma_{\mathrm{e}}+\gamma_{\mathrm{r}}+\rho_{\mathrm{u}}\left(1+\frac{1}{1-\delta}\right)\right. \\
& \left.+\lambda \mathrm{s}\left(1+\frac{\rho_{\mathrm{u}}}{1-\delta}\right)+\frac{\lambda \mathrm{T}}{1-\delta}(1-\lambda \mathrm{s})\right] .
\end{aligned}
$$

Since $\lambda \tau_{t}$ is usually orders of magnitude less than $\rho_{u}$, Eq. 14 can be written as

$$
0<s \leq \tau_{t}, u_{T}(s)=q_{0}+\left(1-q_{0}\right)\left(\gamma_{e}+\gamma_{r}+\rho_{u} \frac{2-\delta}{1-\delta}+\lambda s+\frac{\lambda T}{1-\delta}\right) .
$$

Using the same method and approximations, we can rewrite Eq. 10 as

$$
\tau_{t}<s \leq \theta, u_{R}(s)=\gamma_{e}+\gamma_{r}+\rho_{u} \frac{2-\delta}{1-\delta}+\lambda s+\frac{\lambda T}{1-\delta} .
$$

Finally, Eq. 12 can be written as

$$
\theta<s \leq \mathrm{T}, \mathrm{u}(\mathrm{s})=\gamma_{\mathrm{r}}+\frac{\rho_{\mathrm{u}}}{1-\delta}+\lambda\left(\mathrm{s}+\frac{\delta \mathrm{T}}{\mathrm{l}-\delta}\right)
$$




\section{Simplification of Equations}

To derive the unavailability formulas for the various systems, the basic unavailability equations must be simplified. Equation 15 can be written as

$$
0<s \leq \tau_{t}, u_{T}(s)=A+a^{\prime} \Gamma
$$

where

$$
A=q_{0}+\left(1-q_{0}\right)\left(\gamma_{e}+\gamma_{r}+\rho_{u} \frac{2-\delta}{1-\delta}\right)
$$

and

$$
a=\left(1-q_{0}\right) \lambda \text {. }
$$

The $\lambda s$ term was dropped since the $\lambda \tau_{t}$ contribution to $u_{T}(s)$ is small compared to the rest of the terms. This can also be interpreted as if random failures are immediately repaired during the testing and repair periods. Also, since $\delta \ll 1$, the $\left(1-q_{0}\right) \lambda /(1-\delta)$ term was simplified to $\left(1-q_{0}\right) \lambda$ in the expression for $a$.

Using the same logic, we can write Eq. 16 as

$$
\tau_{\mathrm{t}}<\mathrm{s} \leq \mathrm{e}, \mathrm{u}_{\mathrm{R}}(\mathrm{s})=\mathrm{B}+\mathrm{bT},
$$

where

$$
B=\gamma_{e}+\gamma_{r}+\rho_{u} \frac{2-\delta}{l-\delta}
$$

and

$$
\mathrm{b}=\lambda \text {. }
$$

For the operational standby phase, the unavailability is

$$
\theta<s \leq T, u(s)=C+c T+D^{\prime} \lambda s,
$$

where

$$
\begin{aligned}
& c=\gamma_{r}+\frac{\rho_{u}}{1-\delta}, \\
& c=\frac{\lambda \delta}{1-\delta},
\end{aligned}
$$

and

$$
D^{\prime}=1.0
$$


Equation 20 can be further simplified by setting $c=0$ and redefining the $D^{\prime}$ terms to account for the loss. At $s=\mathrm{T} / 2$,

$$
\bar{x}(\mathrm{~T} / 2)=\mathrm{C}+\mathrm{C} T+\mathrm{D}^{\prime} \lambda \mathrm{T} / 2=\mathrm{C}+\mathrm{D} \lambda \mathrm{T} / 2,
$$

which leads to

$$
D=\frac{1+6}{1-\delta}
$$

So,

$$
\theta<s \leq T, u(s)=C+D \lambda s .
$$

This preserves the average unavailability in the interval and makes the term easier to work with.

C. frerage Unavailability

A $1 / 1$ system has one component that must always be available for the system to br available. For this system, the average unavailability is given by

$$
\bar{U}=\frac{1}{T} \int_{0}^{\tau_{t}} u_{r_{1}}(s) d s+\frac{1}{T} \int_{\tau_{t}}^{\theta} u_{R}(s) d s+\frac{1}{T} \int_{\theta}^{T} u(s) d s .
$$

Integrating and rearranging terms yield

$$
\vec{U}=(1 / T)\left(\tau_{t} A^{1}+\tau_{r} B^{\prime}\right)+\tau_{t} a+\tau r^{b}+C+(1 / T)\left(T^{2}-\theta^{2}\right) D \lambda / 2,
$$

where

$$
A^{\prime}=A-C \text { and } B^{\prime}=B-C \text {. }
$$

Since $\theta \ll T, T^{2}-\theta^{2} \approx T^{2}$ and Eq. 23 can be written

$$
\bar{U}=(1 / T)\left(\tau_{t} A^{\prime}+\tau_{r} B^{\prime}\right)+\tau_{t}{ }^{a}+\tau_{r} b+C+D \lambda T / 2 .
$$

D. Optimum Test Interval

The optimum test interval is the solution of $\mathrm{d} \overline{\mathrm{U}} / \mathrm{dT}=0$ for $\mathrm{T}_{0}$ or

$$
-\left(1 / T_{0}^{2}\right)\left(\tau_{t} A^{\prime}+\tau_{r} B^{\prime}\right)+D \lambda / 2=0
$$

so that

$$
T_{0}=\sqrt{\frac{2}{D \lambda}\left(\tau_{t} A^{\prime}+\tau_{r} B^{\prime}\right)} .
$$


These results follow directly from the concepts developed in Chapter I, Eqs. 5 and 6.

E. Summary

The terms used in this work were discussed in Sec. II.A. Ther were then used to develop the basic unavailability equations for a component in each of its three test-interval periods. From these equations, the average unavailability equation for a $1 / 1$ system was calculated. This also allowed the calculation of the optimum test interval.

The $1 / 1$ system equations form the cornerstone on which more complicated system equatiors can be built. The unavailability equations derived for a component in its three phases of operation are sunmarjzed in Table II.

TABLE II. Unavailability Equations during Testing, Repair, and Operational-standby Periods

\begin{tabular}{llc}
\hline Period & Time & Unavailability Equation \\
\hline Testing & $0<s \leq \tau_{t}$ & $A+a T$ \\
Repair & $\tau_{t}<s \leq \theta$ & $B+b T$ \\
Stinddby & $\theta<s \leq T$ & $C+D \lambda s$
\end{tabular}

where

$$
\begin{aligned}
& A=q_{0}+\left(1-q_{0}\right)\left\{\gamma_{e}+\gamma_{r}+\rho_{u}[(2-\delta) /(1-\delta)]\right\} \\
& a=\left(1-q_{0}\right) \lambda, \\
& B=\gamma_{e}+\gamma_{r}+\rho_{u}[(2-\delta) /(1-\delta)] \\
& b=\lambda \\
& C=\gamma_{r}+\rho_{u} /(1-\delta)
\end{aligned}
$$

and

$$
D=(1+\delta) /(1-\delta)
$$




\section{SYSTEM UNAVAILABILITY EQUATIONS}

A. Introduction

This chapter will introduce and develop the average unavailability equations for all $\mathrm{m} / \mathrm{n}$ single-component redundancy systems with $1 \mathrm{~s} \mathrm{~m}$ $\mathrm{n} \leq 4$, for the random, uniformly staggered, and nearly simultaneous testing schemes.

\section{B. Random Testing Scheme}

In the random testing scheme, the components are tested at randornly shifted times throughout the interval. The average unavailability for any $\mathrm{m} / \mathrm{n}$ system can be given by the expression

$$
\bar{U}_{m / n}=\sum_{k=n-m+1}^{n}\left(\begin{array}{l}
n \\
k
\end{array}\right) \bar{U}^{k}(1-\bar{U})^{n-k},
$$

where $\bar{U}$ is the $\bar{U}$ of Eq. 24 and $\left(\begin{array}{l}n \\ k\end{array}\right)$ is the binomial coefficient $n ! /[(n-k) ! k !]$.

All systems tested in this random scheme have the same optimum test interval. This interval, $T_{0}$, is defined by Eq. 25 and is thought of as being the optimum average test interval.

The equations for the average unavailability of any $m / n(1 \leq m \leq n \leq 4)$ system tested using the random testing scheme are lisied in Table III.

TABLE III. Average Unavailability Equations for $\mathrm{m} / \mathrm{n}$ Systems with Random Testing Scheme

\begin{tabular}{ll|ll}
\hline $\mathrm{m} / \mathrm{n}$ & \multicolumn{1}{c|}{$\overline{\mathrm{U}}_{\mathrm{m} / \mathrm{n}}$} & $\mathrm{m} / \mathrm{n}$ & $\overline{\mathrm{U}}_{\mathrm{m} / \mathrm{n}}$ \\
\hline $1 / 2$ & $\overline{\mathrm{U}}^{2}$ & $1 / 4$ & $\overline{\mathrm{U}}$ \\
$2 / 2$ & $2 \overline{\mathrm{U}}-\overline{\mathrm{U}}^{2}$ & $2 / 4$ & $4 \overline{\mathrm{U}}^{3}-3 \overline{\mathrm{U}}^{4}$ \\
$1 / 3$ & $\overline{\mathrm{U}}^{3}$ & $3 / 4$ & $6 \overline{\mathrm{U}}^{2}-8 \overline{\mathrm{U}}^{3}+3 \overline{\mathrm{U}}^{4}$ \\
$2 / 3$ & $3 \overline{\mathrm{U}}^{2}-2 \overline{\mathrm{U}}^{3}$ & $4 / 4$ & $4 \overline{\mathrm{U}}-6 \overline{\mathrm{U}}^{2}+4 \overline{\mathrm{U}}^{3}-\overline{\mathrm{U}}^{4}$ \\
$3 / 3$ & $3 \overline{\mathrm{U}}-3 \overline{\mathrm{U}}^{2}+\overline{\mathrm{U}}^{3}$ & & \\
where & & \\
$\overline{\mathrm{U}}=(1 / \mathrm{T})\left[\tau_{\mathrm{t}}(\mathrm{A}-\mathrm{C})+\tau_{\mathrm{r}}(\mathrm{B}-\mathrm{C})\right]+\tau_{\mathrm{t}^{\mathrm{a}}}+\tau_{\mathrm{r}} \mathrm{b}+\mathrm{C}+\mathrm{D} \lambda \mathrm{T} / 2$. & (Eq. 24) \\
\hline
\end{tabular}

\section{Uniformly Staggered Testing Scheme}

In the uniformly staggered testing scheme, the components are tested at times $s=0, T / n, 2 T / n, \ldots,(n-1) T / n$. The average unavailability equa tions for a $1 / 1$ system are equivalent, no matter which testing scheme is used. Thus, the equations fur systems with two or more redundancies will be presented. 


\section{Two-component Systems}

If two components are in the system, it can be either a $1 / 2$ system (only one seed be available) or a $2 / 2$ system (both available).

a. One-uut-of-two (1/2) System. For a $1 / 2$ system, the unavailability is the probability that one component is unavailable times the probability that the second component is also unavailable. This product is simply $\mathrm{U}(\mathrm{s}) \mathrm{U}(\mathrm{s}+\mathrm{T} / 2)$ for each of the three periods or

$$
\begin{aligned}
& 0<s \leq \tau_{t}, U(s)=(f+a T)(C+D \lambda T / 2), \\
& \tau_{t}<s \leq A, U(s)=(B+b T)(C+D \lambda \cdot T / 2),
\end{aligned}
$$

and

$$
A<s s T / 2, U(s)=(C+D \lambda s)[C+D \lambda(s+T / 2)] .
$$

Since the durations of both the test $\left(\tau_{t}\right)$ and the repair $\left(\tau_{r}\right)$ are short compared to $\mathrm{T}$, the unavailability of the component that is on operational standby is held constant while the first component is undergoing test and repair.

The average unavailability for this system is

$$
\overrightarrow{\mathrm{U}}_{1 / 2}=\frac{2}{\mathrm{~T}} \int_{0}^{\mathrm{T} / 2} \mathrm{U}(\mathrm{s}) \mathrm{U}(\mathrm{s}+\mathrm{T} / 2) \mathrm{d} \mathrm{s}
$$

Note that the integration is performed over only half the intervai since, when the first component reaches time $s=\mathrm{T} / 2$, the second component begins testing.

Integrating Eq. 30 and gathering terms yields

$$
\begin{aligned}
\overline{\mathrm{U}}_{1 / 2}= & (2 \mathrm{C} / \mathrm{T})\left(\tau_{\mathrm{t}^{\prime}} \mathrm{A}^{\prime}+{ }_{\mathrm{r}} \mathrm{B}^{\prime}\right)+2 \mathrm{C}\left(\tau_{\mathrm{t}} \mathrm{a}+\tau_{\mathrm{r}} \mathrm{b}+\mathrm{C} / 2\right)+\mathrm{D} \lambda\left(\tau_{\mathrm{t}} \mathrm{A}^{\prime}+\tau_{\mathrm{r}} \mathrm{B}^{\prime}\right) \\
& +\mathrm{D} \lambda \mathrm{T}\left(\tau_{t^{\mathrm{a}}}+\tau_{\mathrm{r}} \mathrm{b}\right)+(2 / \mathrm{T})\left(2 \mathrm{CD} \lambda+\mathrm{D}^{2} \lambda^{\prime} \mathrm{T} / 2\right)\left(\mathrm{T}^{2} / 4-\theta^{2}\right) \\
& +(2 \mathrm{D} \lambda / 3 \mathrm{~T})\left(\mathrm{T}^{3} / \mathrm{b}-\theta^{3}\right) .
\end{aligned}
$$

If $T=720 \mathrm{~h}, \tau_{t}=2 \mathrm{~h}$, and $\tau_{r}=15 \mathrm{~h}$, Tables IV-VI show the errors incurred by making various approximations. For $\mathrm{n}>1$ and $\mathrm{k}=2,3,4$, approximating $(\mathrm{T} / \mathrm{k})^{\mathrm{n}}-\mathrm{e}^{\mathrm{n}}$ by $(\mathrm{T} / \mathrm{k})^{\mathrm{n}}$ gives rise to an error of less than $1 \%$ in each case. These approximaiions will be used in the applicable system unavailability equations. 
TABLE IV. Errors in $(T / 2)^{n}-\theta^{n} \approx(T / 2)^{n}$ Approximation

\begin{tabular}{llcc}
\hline $\mathrm{n}$ & $(\mathrm{T} / 2)^{\mathrm{n}}$ & $\theta^{\mathrm{n}}$ & $\begin{array}{c}\text { Percent } \\
\text { Errora }\end{array}$ \\
\hline 1 & 360 & 17 & 4.96 \\
2 & $1.30 \times 10^{5}$ & $2.89 \times 10^{2}$ & 0.22 \\
3 & $4.67 \times 10^{7}$ & $4.91 \times 10^{3}$ & $0.0 \mathrm{i}$ \\
4 & $1.68 \times 10^{10}$ & $8.35 \times 10^{4}$ & $4.97 \times 10^{-4}$ \\
5 & $6.25 \times 10^{12}$ & $1.42 \times 10^{6}$ & $2.35 \times 10^{-5}$ \\
\hline
\end{tabular}

a $\frac{\theta^{n}}{(T / 2)^{n}-\theta^{n}} \times 100 \%$

TABLE V. Errors in $(T / 3)^{n}-\theta^{n} \approx(T / 3)^{n}$ Approximation

\begin{tabular}{lccc}
\hline $\mathrm{n}$ & $(\mathrm{T} / 3)^{\mathrm{n}}$ & $\rho^{\mathrm{n}}$ & $\begin{array}{c}\text { Percent } \\
\text { Error }\end{array}$ \\
\hline 1 & 240 & 17 & 7.62 \\
2 & $5.76 \times 10^{4}$ & $2.89 \times 10^{2}$ & 0.504 \\
3 & $1.38 \times 10^{7}$ & $4.91 \times 10^{3}$ & $3.56 \times 10^{-2}$ \\
4 & $3.32 \times 10^{9}$ & $8.35 \times 10^{4}$ & $2.52 \times 10^{-3}$ \\
5 & $7.96 \times 10^{11}$ & $1.42 \times 10^{6}$ & $1.78 \times 10^{-4}$ \\
\hline
\end{tabular}

a

$\frac{\theta^{n}}{(T / 3)^{n}-\theta^{n}} \times 100 \%$

TABLE VI. Errors in $(T / 4)^{\mathrm{n}}-\theta^{\mathrm{n}} \sim(\mathrm{T} / 4)^{\mathrm{n}}$ Approximation

\begin{tabular}{cccc}
\hline $\mathrm{n}$ & $(\mathrm{T} / 4)^{\mathrm{n}}$ & $\theta^{\mathrm{n}}$ & $\begin{array}{c}\text { Percent } \\
\text { Error }\end{array}$ \\
\hline 1 & 100 & 17 & 10.43 \\
2 & $3.24 \times 10^{4}$ & $2.89 \times 10^{2}$ & 0.90 \\
3 & $5.83 \times 10^{6}$ & $4.91 \times 10^{3}$ & 0.084 \\
4 & $1.05 \times 10^{9}$ & $8.35 \times 10^{4}$ & $7.96 \times 10^{-3}$ \\
5 & $1.89 \times 10^{11}$ & $1.42 \times 10^{6}$ & $7.51 \times 10^{-4}$ \\
\hline
\end{tabular}

a

$\frac{\theta^{n}}{(T / 4)^{n}-\theta^{n}} \times 100 \%$ 
Equation 31 can be simplified to

$$
\begin{aligned}
\bar{U}_{1 / 2}= & (2 \mathrm{C} / \mathrm{T})\left(\tau_{t} A^{\prime}+{ }_{r} B^{\prime}\right)+2 C\left(\tau_{t} a+\tau_{r} b+C / 2\right)+D \lambda\left(\tau_{t} A^{\prime}+\tau_{r} B^{\prime}\right) \\
& +D \lambda T\left(\tau_{t} a+\tau_{r} b+C\right)+(5 / 24) D^{2} \lambda^{2} T^{2} .
\end{aligned}
$$

b. Two-out-of-two (2/2) System. In the $2 / 2$ system, it is easier to think in terms of the availability of the system, $\left(1-U_{2} / 2\right)$. In this case, the system is available only if both components are available:

$$
1-U_{2 / 2}=\left(1-U_{1}\right)\left(1-U_{2}\right)
$$

or

$$
\mathrm{U}_{2 / 2}=1-\left(1-\mathrm{U}_{1}\right)\left(1-\mathrm{U}_{2}\right)
$$

So for this system,

$$
\begin{aligned}
& 0<s \leq \tau_{t}, U(s)=1-[1-(A+a T)][1-(C+D \lambda T / 2)] \\
& \tau_{t}<s \leq \theta, U(s)=1-[1-(B+b T)][1-(C+D \lambda T / 2)]
\end{aligned}
$$

and

$$
\theta<s \leq T / 2, U(s)=1-[1-(C+D \lambda s)]\{1-[C+D \lambda(s+T / 2)]\}
$$

The average unavailability is then given by

$$
\begin{aligned}
\bar{U}_{2 / 2}= & \frac{2(1-C)}{T}\left(\tau_{t} A^{\prime}+\tau_{r} B^{\prime}\right)+1+2(1-C)\left(\tau_{t} a+\tau_{r} b\right) \\
& -D \lambda\left(\tau_{t} A^{\prime}+\tau_{r} B^{\prime}\right)-(C-1)^{2}-D \lambda T\left(\tau_{t} a+\tau_{r} b+C-1\right) \\
& -(5 / 24) D^{2} \lambda^{2} T^{2} .
\end{aligned}
$$

\section{Three-component Systerns}

If there are three components in the system, it can be a $1 / 3,2 / 3$, or $3 / 3$ system. The average unavailability is given by

$$
\overline{\mathrm{U}}=\frac{3}{\mathrm{~T}} \int_{0}^{\mathrm{T} / 3} \mathrm{U}(\mathrm{s}) \mathrm{d} \mathrm{s} .
$$

a. One-out-of-three (1/3) System. For a $1 / 3$ system, the unavailability is the product of the unavailability of the three components:

$$
0<s \leq \tau_{t}, U(s)=(A+a T)(C+D \lambda T / 3)(C+2 D \lambda T / 3)
$$




$$
T_{t}<s \leq \theta, U(s)=(B+b T)(C+D \lambda T / 3)(C+2 D \lambda T / 3)
$$

and

$e<s \leq T / 3, U(s)=(C+D \lambda s)[C+D \lambda(s+T / 3)][C+D \lambda(s+2 T / 3)]$.

Again, since the durations of the test anc lepair periods are much shorter than $\mathrm{T} / 3$, the unavailabilities of the components on operational standby can be treated as being constant.

The average unavailability for a $1 / 3$ system is

$$
\begin{aligned}
\overline{\mathrm{U}}_{1 / 3}= & \left(3 \mathrm{C}^{2} / T\right)\left(\tau_{t} A^{\prime}+\tau_{r} B^{\prime}\right)+3 C^{2}\left(\tau_{t} a+\tau_{r} b\right)+3 C D \lambda\left(\tau_{t} A^{\prime}+\tau_{r} B^{\prime}\right) \\
& +C^{3}+D \lambda T\left[3 C\left(\tau_{t} a+\tau_{r} b\right)+(2 / 3) D \lambda\left(\tau_{t} A^{\prime}+\tau_{r} B^{\prime}\right)+(3 / 2) C^{2}\right] \\
& +(2 / 3) D^{2} \lambda^{2} T^{2}\left(\tau_{t} a+\tau_{r} b+C\right)+(1 / 12) D^{3} \lambda^{3} T^{3} .
\end{aligned}
$$

b. Two-out-of-three (2/3) System. A $2 / 3$ system is unavailable when any two components are unavailable:

$$
\begin{aligned}
\mathrm{U}_{2 / 3} & =\mathrm{U}_{1} \mathrm{U}_{2}\left(1-\mathrm{U}_{3}\right)+\mathrm{U}_{1} \mathrm{U}_{3}\left(1-\mathrm{U}_{2}\right)+\mathrm{U}_{2} \mathrm{U}_{3}\left(1-\mathrm{U}_{1}\right)+\mathrm{U}_{1} \mathrm{U}_{2} \mathrm{U}_{3} \\
& =\mathrm{U}_{1} \mathrm{U}_{2}+\mathrm{U}_{1} \mathrm{U}_{3}+\mathrm{U}_{2} \mathrm{U}_{3}-2 \mathrm{U}_{1} \mathrm{U}_{2} \mathrm{U}_{3} .
\end{aligned}
$$

interval are

The unavailabilities in each of the periods in the test

$$
\begin{array}{rl}
0<s & s T_{t}, U(s)=(A+a T)(C+D \lambda T / 3)+(A+F T)(C+2 D \lambda T / 3) \\
& +(C+D \lambda T / 3)(C+2 D \lambda T / 3)-2(A+a T)(C+D \lambda T / 3)(C+2 D \lambda T / 3) \\
T_{t}<s \leq \theta, U(s)=(B+b T)(C+D \lambda T / 3)+(B+b T)(C+2 D \lambda T / 3) \\
\quad+(C+D \lambda T / 3)(C+2 D \lambda T / 3)-2(B+b T)(C+D \lambda T / 3)(C+2 D \lambda T / 3)
\end{array}
$$

and

$$
\begin{aligned}
\theta< & s \leq T / 3, U(s)=(C+D \lambda s)[C+D \lambda(s+T / 3)]+(C+D \lambda s) \\
\cdot & {[C+D \lambda(s+2 T / 3)]+[C+D \lambda(s+T / 3)][C+D \lambda(s+2 T / 3)] } \\
& -2(C+D \lambda s)[C+D \lambda(s+T / 3)][C+D \lambda(s+2 T / 3)] .
\end{aligned}
$$

Integrating and rearranging terms give the average unavailability of the system 


$$
\begin{aligned}
\bar{U}_{2 / 3}= & (6 C / T)(1-C)\left(\tau_{t} A^{\prime}+\tau_{r} B^{\prime}\right)+6 C(1-C)\left(\tau_{t a}+\tau_{r} b\right) \\
& +3 D \lambda(1-2 C)\left(\tau_{t} A^{\prime}+\tau_{r} B^{\prime}\right)+3 C^{2}-2 C^{3} \\
& +D \lambda T\left\{3(1-2 C)\left(\tau_{t} a+\tau_{r} b\right)-(4 / 3) D \lambda\left(\tau_{t} A^{\prime}+\tau_{r} B^{\prime}\right)+3 C(1-C)\right\} \\
& -(2 / 3) D^{2} \lambda^{2} T^{2}\left[2\left(\tau_{t} a+\tau_{r} b+C\right)-1\right]-(1 / 6) D^{3} \lambda^{3} T^{3} .
\end{aligned}
$$

c. Three-out-of-three (3/3) System. For a $3 / 3$ system, it again becomes more practical to think in terms of availability:

$$
1-U_{3 / 3}=\left(1-U_{1}\right)\left(1-U_{2}\right)\left(1-U_{3}\right)
$$

or

$$
\mathrm{U}_{3 / 3}=1-\left(1-\mathrm{U}_{1}\right)\left(1-\mathrm{U}_{2}\right)\left(1-\mathrm{U}_{3}\right)
$$

The unavailability is given by

$$
\begin{aligned}
& 0<s \leq \tau_{t}, U(s)=1-[1-(A+a T)][1-(C+D \lambda T / 3)][1-(C+2 D \lambda T / 3)] \\
& \tau_{t}<s \leq \theta, U(s)=1-[1-(B+b T)][1-(C+D \lambda T / 3)][1-(C+2 D \lambda T / 3)] \\
& \text { and }
\end{aligned}
$$

$$
\begin{aligned}
\theta< & s \leq T / 3, U(s)=1-[1-(C+D \lambda s)]\{1-[C+D \lambda(s+T / 3)]\} \\
\cdot & \{1-[C+D \lambda(s+2 T / 3)]\} .
\end{aligned}
$$

And the average unavailability of the systern is

$$
\begin{aligned}
\bar{U}_{3 / 3}= & (3 / T)(C-1)^{2}\left(\tau_{t} A^{\prime}+\tau_{r} B^{\prime}\right)+3(C-1)^{2}\left(\tau_{t} a+\tau_{r} b\right) \\
& +1+(C-1)^{3}+3(C-1) D \lambda\left(\tau_{t} A^{\prime}+\tau_{r} B^{\prime}\right) \\
& +3 D \lambda T\left[(2 / 9) D \lambda\left(\tau_{t} A^{\prime}+\tau_{r} B^{\prime}\right)+(C-1)\left(\tau_{t} a+\tau_{r} b\right)+(1 / 2)(C-1)^{2}\right] \\
& +(2 / 3) D^{2} \lambda^{2} T^{2}\left(\tau_{t} a+\tau_{r} b+C-1\right)+(1 / 12) D^{3} \lambda^{3} T^{3} .
\end{aligned}
$$

\section{Four-component Systems}

When four components are in a system, the system can be either $1 / 4,2 / 4,3 / 4$, or $4 / 4$. The average unavailability is given by

$$
\overline{\mathrm{U}}=\frac{4}{\mathrm{~T}} \int_{u}^{\mathrm{T} / 4} \mathrm{U}(\mathrm{s}) \mathrm{d} \mathrm{s} .
$$


a. One-out-of-four (1/4) System. For a $1 / 4$ system, the unavailabilities during testing, repair and standby are given by

$0<s \leq \tau_{t}, U(s)=(A+a T)(C+D \lambda T / 4)(C+D \lambda T / 2)(C+3 D \lambda T / 4)$,

$\tau_{t}<s \leq \theta, U(s)=(B+b T)(C+D \lambda T / 4)(C+D \lambda T / 2)(C+3 D \lambda T / 4)$,

and

$$
\begin{aligned}
\theta<s \leq T / 4, U(s)= & (C+D \lambda s)[C+D \backslash(s+T / 4)][C+D \lambda(s+T / 2)] \\
\cdot & {[C+D \lambda(s+3 T / 4)] . }
\end{aligned}
$$

The average unavailability for this system is

$$
\begin{aligned}
\overline{\mathrm{U}}_{1 / 4} & =\left(4 \mathrm{C}^{3} / T\right)\left(\tau_{t} A^{\prime}+\tau_{r} B^{\prime}\right)+C^{4}+4 C^{3}\left(\tau_{t} a+\tau_{r} b\right)+6 C^{2} D \lambda\left(\tau_{t} A^{\prime}+\tau_{r} B^{\prime}\right) \\
& +C D \lambda T\left[6 C\left(\tau_{t} a+\tau_{r} b\right)+(11 / 4) D \lambda\left(\tau_{t} A^{\prime}+\tau_{r} B^{\prime}\right)+2 C^{2}\right] \\
& +(1 / 8) D^{2} \lambda^{2} T^{2}\left[22 C\left(\tau_{t} a+\tau_{r} b\right)+3 D \lambda\left(\tau_{t} A^{\prime}+{ }^{2}{ }_{r} B^{\prime}\right)+11 C^{2}\right] \\
& +(3 / 8) D^{3} \lambda^{3} T^{3}\left(\tau_{t} a+\tau_{r} b+C\right)+(251 / 7680) D^{4} \lambda^{4} T^{4}
\end{aligned}
$$

b. Two-out-of-four (2/4) System. In a $2 / 4$ system, any three of the components being down causes the system to be unavailable:

$$
\begin{aligned}
\mathrm{U}_{2 / 4}= & \mathrm{U}_{1} \mathrm{U}_{2} \mathrm{U}_{3}\left(1-\mathrm{U}_{4}\right)+\mathrm{U}_{1} \mathrm{U}_{2} \mathrm{U}_{4}\left(1-\mathrm{U}_{3}\right)+\mathrm{U}_{1} \mathrm{U}_{3} \mathrm{U}_{4}\left(1-\mathrm{U}_{2}\right) \\
& +\mathrm{U}_{2} \mathrm{U}_{3} \mathrm{U}_{4}\left(1-\mathrm{U}_{1}\right)+\mathrm{U}_{1} \mathrm{U}_{2} \mathrm{U}_{3} \mathrm{U}_{4} \\
= & \mathrm{U}_{1} \mathrm{U}_{2} \mathrm{U}_{3}+\mathrm{U}_{1} \mathrm{U}_{2} \mathrm{U}_{4}+\mathrm{U}_{1} \mathrm{U}_{3} \mathrm{U}_{4}+\mathrm{U}_{2} \mathrm{U}_{3} \mathrm{U}_{4}-3 \mathrm{U}_{1} \mathrm{U}_{2} \mathrm{U}_{3} \mathrm{U}_{4} .
\end{aligned}
$$

Thus, the unavailability equations for the three periods in the test interval are

$$
\begin{aligned}
0< & s \tau_{t}, U(s)=(A+a T)(C+D \lambda T / 4)(C+D \lambda T / 2)+(A+a T)(C+D \lambda T / 4) \\
& \cdot(C+3 D \lambda T / 4)+(A+a T)(C+D \lambda T / 2)(C+3 D \lambda T / 4)+(C+D \lambda T / 4) \\
& \cdot(C+D \lambda T / 2)(C+3 D \lambda T / 4)-3(A+a T)(C+D \lambda T / 4)(C+D \lambda T / 2) \\
& \cdot(C+3 D \lambda T / 4) \\
\tau_{t}< & s \theta, U(s)=(B+b T)(C+D \lambda T / 4)(C+D \lambda T / 2)+(B+b T)(C+D \lambda T / 4) \\
& \cdot(C+D \lambda T / 2)+(B+b T)(C+D \lambda T / 4)(C+3 D \lambda T / 4)+(C+D \lambda T / 4) \\
& \cdot(C+D \lambda T / 2)(C+3 D \lambda T / 4)-3(B+b T)(C+D \lambda T / 4)(C+D \lambda T / 2) \\
& \cdot(C+3 D \lambda T / 4)
\end{aligned}
$$


and

$$
\begin{aligned}
\theta< & s \leq T / 4, U(s)=(C+D \lambda s)[C+D \lambda(s+T / 4)][C+D \lambda(s+T / 2)] \\
+ & (C+D \lambda s)[C+D \lambda(s+T / 4)][C+D \lambda(s+3 T / 4)]+(C+D \lambda s) \\
\cdot & {[C+D \lambda(s+T / 2)][C+D \lambda(s+3 T / 4)]+[C+D \lambda(s+T / 4)] } \\
\cdot & {[C+D \lambda(s+T / 2)][C+D \lambda(s+3 T / 4)]-3(C+D \lambda s)[C+D \lambda(s+T / 4)] } \\
\cdot & {[C+D \lambda(s+T / 2)][C+D \lambda(s+3 T / 4)] . }
\end{aligned}
$$

Integrating and rearranging terms give the average unavailability of a $2 / 4$ system as

$$
\begin{aligned}
& \bar{U}_{2 / 4}=\left(12 C^{2} / T\right)(1-C)\left(\tau_{t} A^{\prime}+\tau_{r^{\prime}} B^{\prime}\right)+6 C D \lambda(2-3 C)\left(\tau t A^{\prime}+\tau_{r} B^{\prime}\right) \\
& +12 C^{2}(1-C)\left(\tau_{t} a+\tau_{r} b\right)-3 C^{4}+4 C^{3} \\
& +D \lambda T\left\{(11 / 4)(1-3 C) D \lambda\left(\tau_{t} A^{1}+{ }^{\top} r^{B^{\prime}}\right)+6 C\left[(2-3 C)\left(\tau_{t} a+{ }^{\top} r^{b}\right)\right.\right. \\
& +C(1-C)]\}+(1 / 8) D^{2} \lambda^{2} T^{2}\left[22(1-3 C)\left(\tau_{t} a+T^{b} b\right)\right. \\
& \left.-9 D \lambda\left(\tau t A^{\prime}+\tau_{r} B^{\prime}\right)+11 C(2-3 C)\right] \\
& -(3 / 8) D^{3} \lambda^{3} T^{3}\left[3\left(\tau_{t a}+{ }^{\top} r^{b}+C\right)-1\right]-(251 / 2560) D^{4} \lambda^{4} T^{4} \text {. }
\end{aligned}
$$

c. Three-out-of-four (3/4) System. In a $3 / 4$ system, if any two components are down, the system is down. In terins of the availability, this becomes

$$
\begin{aligned}
1-\mathrm{U}_{3 / 4}= & \left(1-\mathrm{U}_{1}\right)\left(1-\mathrm{U}_{2}\right)\left(1-\mathrm{U}_{3}\right) \mathrm{U}_{4}+\left(1-\mathrm{U}_{1}\right)\left(1-\mathrm{U}_{2}\right)\left(1-\mathrm{U}_{4}\right) \mathrm{U}_{3} \\
& +\left(1-\mathrm{U}_{1}\right)\left(1-\mathrm{U}_{3}\right)\left(1-\mathrm{U}_{4}\right) \mathrm{U}_{2}+\left(1-\mathrm{U}_{2}\right)\left(1-\mathrm{U}_{3}\right)\left(1-\mathrm{U}_{4}\right) \mathrm{U}_{1} \\
& +\left(1-\mathrm{U}_{1}\right)\left(1-\mathrm{U}_{2}\right)\left(1-\mathrm{U}_{3}\right)\left(1-\mathrm{U}_{4}\right)
\end{aligned}
$$

or

$$
\begin{aligned}
\mathrm{U}_{3 / 4}= & \mathrm{U}_{1} \mathrm{U}_{2}+\mathrm{U}_{1} \mathrm{U}_{3}+\mathrm{U}_{1} \mathrm{U}_{4}+\mathrm{U}_{2} \mathrm{U}_{3}+\mathrm{U}_{2} \mathrm{U}_{4}+\mathrm{U}_{3} \mathrm{U}_{4}-2 \mathrm{U}_{1} \mathrm{U}_{2} \mathrm{U}_{3} \\
& -2 \mathrm{U}_{1} \mathrm{U}_{2} \mathrm{U}_{4}-2 \mathrm{U}_{1} \mathrm{U}_{3} \mathrm{U}_{4}-2 \mathrm{U}_{2} \mathrm{U}_{3} \mathrm{U}_{4}+3 \mathrm{U}_{1} \mathrm{U}_{2} \mathrm{U}_{3} \mathrm{U}_{4},
\end{aligned}
$$




$$
\begin{array}{rl}
0< & s T_{t}, U(s)=(A+a T)(C+D \lambda T / 4)+(A+a T)(C+D \lambda T / 2)+(A+a T) \\
& \cdot(C+3 D \lambda T / 4)+(C+D \lambda T / 4)(C+D \lambda T / 2)+(C+D \lambda T / 4)(C+3 D \lambda T / 4) \\
& +(C+D \lambda T / 2)(C+3 D \lambda T / 4)-2(A+a T)(C+D \lambda T / 4)(C+D \lambda T / 2) \\
& -2(A+a T)(C+D \lambda T / 4)(C+3 D \lambda T / 4)-2(A+a T)(C+D \lambda T / 2)(C+3 D \lambda T / 4) \\
& -2(C+D \lambda T / 4)(C+D \lambda T / 2)(C+3 D \lambda T / 4)+3(A+a T)(C+D \lambda T / 4) \\
& \cdot(C+D \lambda T / 2)(C+3 D \lambda T / 4), \\
T_{t} & s \leq \theta, U(s)=(B+b T)(C+D \lambda T / 4)+(B+b T)(C+D \lambda T / 2)+(B+b T) \\
& \cdot(C+3 D \lambda T / 4)+(C+D \lambda T / 4)(C+D \lambda T / 2)+(C+D \lambda T / 4)(C+3 D \lambda T / 4) \\
& +(C+D \lambda T / 2)(C+3 D \lambda T / 4)-2(B+b T)(C+D \lambda T / 4)(C+D \lambda T / 2) \\
& -2(B+b T)(C+D \lambda T / 4)(C+3 D \lambda T / t)-2(B+b T)(C+D \lambda T / 2)(C+3 D \lambda T / 4) \\
& -2(C+D \lambda T / 4)(C+D \lambda T / 2)(C+3 D \lambda T / 4)+3(B+b T)(C+D \lambda T / 4) \\
& +(C+D \lambda T / 2)(C+3 D \lambda T / 4),
\end{array}
$$

and

$$
\begin{aligned}
A< & s \leq T / 4, U(s)=(C+D \lambda s)[C+D \lambda(s+T / 4)]+(C+D \lambda s)[C+D \lambda(s+T / 2)] \\
& +(C+D \lambda s)[C+D \lambda(s+3 T / 4)]+[C+D \lambda(s+T / 4)][C+D \lambda(s+T / 2)] \\
& +[C+D \lambda(s+T / 4)][C+D \lambda(s+3 T / 4)]+[C+D \lambda(s+T / 2)] \\
& \cdot[C+D \lambda(s+3 T / 4)]-2(C+D \lambda s)[C+D \lambda(s+T / 4)][C+D \lambda(s+T / 2)] \\
& -2(C+D \lambda s)[C+D \lambda(s+T / 4)][C+D \lambda(s+3 T / 4)]-2[C+D \lambda(s+T / 2)] \\
& \cdot[C+D \lambda(s+3 T / 4)](C+D \lambda s)-2[C+D \lambda(s+T / 4)][C+D \lambda(s+T / 2)] \\
& \cdot[C+D \lambda(s+3 T / 4)]+3(C+D \lambda s)[C+D \lambda(s+T / 4)][C+D \lambda(s+T / 2)] \\
& \cdot[C+D \lambda(s+3 T / 4)] .
\end{aligned}
$$

The average unavailability for this system then becomes 


$$
\begin{aligned}
\overline{\mathrm{U}}_{3 / 4}= & (12 C / T)(C-1)^{2}\left(\tau_{t} A^{\prime}+\tau_{r} B^{\prime}\right)+3 C^{4}-8 C^{3}+6 C^{2}+12 C(1-2 C) \\
& \cdot\left(\tau_{t} a+\tau_{r} b\right)+6 D \lambda(1-4 C)\left(\tau_{t} A^{\prime}+\tau_{r} B^{\prime}\right)+18 C^{2} D \lambda\left(\tau_{t} A^{\prime}+\tau_{r} B^{\prime}\right) \\
& +12 C^{3}\left(\tau_{t} a+\tau_{r} b\right)+D \lambda T\left[6\left(\tau_{t} a+\tau_{r} b\right)(3 C-1)(C-1)\right. \\
& \left.+(11 / 4) D \lambda(3 C-2)\left(\tau_{t} A^{\prime}+\tau r^{\prime}\right)+6 C(C-1)^{2}\right] \\
& +D^{2} \lambda^{2} T^{2}\left[(9 / 8) D \lambda\left(\tau_{t} A^{\prime}+\tau_{r} B^{\prime}\right)+(11 / 4)(3 C-2)\left(\tau_{t} a+\tau_{r} b\right)\right. \\
& +(11 / 8)(3 C-1)(C-1)]+D^{3} \lambda^{3} T^{3}\left[(9 / 8)\left(\tau_{t} a+\tau_{r} b\right)\right. \\
& +(3 / 8)(3 C-2)]+(25 l / 2560) D^{4} \lambda^{4} T^{4} .
\end{aligned}
$$

d. Four-out-of-four (4/4) System. Finally, for a $4 / 4$ system, all the components must be available for the system to be up. In terms of availability,

$$
1-\mathrm{U}_{4 / 4}=\left(1-\mathrm{U}_{1}\right)\left(1-\mathrm{U}_{2}\right)\left(1-\mathrm{U}_{3}\right)\left(1-\mathrm{U}_{4}\right)
$$

or

$$
\begin{aligned}
\mathrm{U}_{4 / 4}=1-\left(1-\mathrm{U}_{1}\right)\left(1-\mathrm{U}_{2}\right)\left(1-\mathrm{U}_{3}\right)\left(1-\mathrm{U}_{4}\right), \\
0<\mathrm{s} \leq \mathrm{i}_{\mathrm{t}}, \mathrm{U}(\mathrm{s})=1-[1-(\mathrm{A}+\mathrm{aT})][1-(\mathrm{C}+\mathrm{D} \lambda \mathrm{T} / 4)][1-(\mathrm{C}+\mathrm{D} \lambda \mathrm{T} / 2)] \\
\cdot[1-(\mathrm{C}+3 \mathrm{D} \lambda \mathrm{T} / 4)], \\
\tau_{\mathrm{t}}<\mathrm{s} \leq \theta, \mathrm{U}(\mathrm{s})=1-[1-(\mathrm{B}+\mathrm{bT})][1-(\mathrm{C}+\mathrm{D} \lambda \mathrm{T} / 4)][1-(\mathrm{C}+\mathrm{D} \lambda \mathrm{T} / 2)] \\
\cdot[1-(\mathrm{C}+3 \mathrm{D} \lambda \mathrm{T} / 4)],
\end{aligned}
$$

and

$$
\begin{gathered}
\theta<s \leq \mathrm{T} / 4, \mathrm{U}(\mathrm{s})=1-[1-(\mathrm{C}+\mathrm{D} \lambda \mathrm{s})]\{1-[\mathrm{C}+\mathrm{D} \lambda(\mathrm{s}+\mathrm{T} / 4)]\} \\
\cdot\{1-[\mathrm{C}+\mathrm{D} \lambda(\mathrm{s}+\mathrm{T} / 2)]\}\{1-[\mathrm{C}+\mathrm{D} \lambda(\mathrm{s}+3 \mathrm{~T} / 4)]\} .
\end{gathered}
$$

The average unavailability for this $4 / 4$ system then becomes

$$
\begin{aligned}
& \overline{\mathrm{U}}_{4 / 4}=(4 / T)(C-1)^{3}\left(\tau_{t} A^{\prime}+{ }^{\top} r B^{\prime}\right)+1-(C-1)^{4}-4(C-1)^{2}\left[(C-1)\left(\tau_{t} a+T_{r} b\right)\right. \\
& \left.+(3 / 2) D \lambda\left(\tau_{t} A^{\prime}+\tau_{r} B^{\prime}\right)\right]-2(C-1) D \lambda T\left[(C-1)^{2}+3(C-1)\left(\tau_{t} a+T_{r} b\right)\right. \\
& \left.+(11 / 8) D \lambda\left(T_{t} A^{\prime}+{ }^{\top} r^{B^{\prime}}\right)\right]-(1 / 8) D^{2} \lambda^{2} T^{2}\left[11(C-1)^{2}+22(C-1)\right. \\
& \left.\left.\cdot\left(\tau_{t} a+\tau_{r} b\right)+3 D \lambda\left(\tau_{t} A^{\prime}+\tau_{r} B^{\prime}\right)\right]-(3 / 8) D^{3}\right){ }^{3} T^{3}\left(\tau_{t} a+\tau_{r} b+C \quad l\right) \\
& -(251 / 7680) D^{4} \lambda^{4} T^{4}
\end{aligned}
$$




\section{Nearly Simultaneous Testing Scheme}

When a system is tested using the nearly simultaneous testing scheme, the components are tested at times $s=0, \tau_{t}, 2 \tau_{t}, \ldots,(n-1)^{\top} t$. Following each test, the repair begins, allowing one test and one repair crew to service all the components. If the testing was done exactly simultaneously, $n$ crews would be needed.

\section{Two-component Systems}

The average unavailability equations for the $1 / 2$ and $2 / 2$ systems are presented in the following sections. Because of the method in which the tests are staggered, the equations will generally be more involved than for the uniformly staggered scheme.

a. One-out-of-two (i/2) System. Fnr a $1 / 2$ system, the unavailability for the system is calculated from

$$
\begin{aligned}
& 0<s \leq \tau_{t}, U(s)=(A+a T)(C+D \lambda T), \\
& \tau_{t}<s \leq 2 \tau_{t}, U(s)=(B+b T)(A+a T), \\
& 2 \tau_{t}<s \leq \theta, U(s)=(B+b T)^{2}, \\
& \theta<s \leq \theta+\tau_{t}, U(s)=(C+D \lambda \theta)(B+b T) .
\end{aligned}
$$

and

$$
f+\tau_{t}<s \leq T, U(s)=(C+\Gamma \lambda s)^{2}
$$

In Eq. 73, unavailability of the second component is assumed to be conslant. This is valid, since $\tau_{t}<T$. This assumption is also made in Eqs. 76 and 77. One criterion that is essential to make this set of equations valid is that $\theta \geq 2 \tau_{t}$ or ${ }^{\top} r \geq T_{t}$. In general, for the $\mathrm{m} / \mathrm{n}$ systems ( $1 \leq m \leq n \leq 4)$ using nearly simultaneous testing. 'T must be greater than $(n-1) \tau_{t}$. This is acceptable based on current testing and repair practice at nuclear power plants.

The average unavailability for this system is

$$
\begin{aligned}
\bar{U}_{1 / 2}= & (1 / T)\left[T_{t}\left(A B+A C+B C-3 C^{2}+B D \lambda \theta\right)+\mu\left(B^{2}-C^{2}\right)\right] \\
& +T_{t}[A(b+D \lambda)+a(B+C)+b(C+D \lambda \theta)]+2 B b \mu+C^{2} \\
& +T\left[\tau_{t} a(b+D \lambda)+b^{2} \mu+C D \lambda\right]+(1 / 3) D^{2} \lambda^{2} T^{2},
\end{aligned}
$$

where $\mu=\theta-2 \tau_{t}$. 
In terms other than $\mathrm{C}^{2}$ we integrated $\mathrm{Eq} .77$ from 0 to $\mathrm{T}$ rather than from $\theta+\tau_{t}$. The errors from this. approximation a re smaller than the errors found in Table VII. For $n>1$, the errors are less than $0.1 \%$. The errors are acceptable.

\begin{tabular}{|c|c|c|c|}
\hline $\mathrm{n}$ & $\mathrm{T}^{\mathrm{n}}$ & $\left(\theta+\tau_{t}\right)^{n}$ & $\begin{array}{l}\text { Percent } \\
\text { Error }\end{array}$ \\
\hline 1 & 720 & 19 & 2.71 \\
\hline 2 & $5.184 \times 10^{5}$ & $3.61 \times 10^{2}$ & $6.97 \times 10^{-2}$ \\
\hline 3 & $3.73 \times 10^{8}$ & $6.86 \times 10^{3}$ & $1.84 \times 10^{-3}$ \\
\hline 4 & $2.69 \times 10^{11}$ & $1.30 \times 10^{5}$ & $4.85 \times 10^{-5}$ \\
\hline 5 & $1.93 \times 10^{14}$ & $2.48 \times 10^{6}$ & $1.28 \times 10^{-6}$ \\
\hline
\end{tabular}

$2 / 2$ system are

b. Two-out-of-two System. The unavailability equations for a

$$
\begin{aligned}
& 0<s \leq{ }_{t}, U(s)=1-[1-(A+a T)][1-(C+D \lambda T)], \\
& { }_{t}<s \leq 2 \top_{t}, U(s)=1-[1-(B+b T)][1-(A+a T)], \\
& 2 \tau_{t}<s \leq \theta, U(s)=1-[1-(B+b T)]^{2}, \\
& \theta<s \leq c+{ }_{t},(1(s)=1-[1-(C+D \lambda \theta)][1-(B+b T)],
\end{aligned}
$$

and

$$
\theta+\tau_{t}<s \leq T, U(s)=1-[1-(C+D \lambda s)]^{2}
$$

The average unavailability for the system is

$$
\begin{aligned}
U_{2 / 2}= & (1 / T)\left[\tau_{t}\left(2 A+2 B-4 C-A B-A C-B C+3 C^{2}-B D \lambda \theta\right)\right. \\
& +\mu(B-C)(2-B-C)]+C(2-C)+\tau_{t}[a(2-B-C)+b(2-A-C) \\
& -D \lambda(A+b D)]+2 \mu b(1-B)+T\left[D \lambda(1-C)-\tau_{t} a(b+D \lambda)-\mu b^{2}\right] \\
& -(1 / 3) D^{2} \lambda^{2} T^{2} .
\end{aligned}
$$

\section{Three-component Systems}

The development of the average unavailability equations for three-component systems is again similar to the development for uniformly staggered testing, exc $\in$ pt for the more complicated test-staggering procedure at the 8 tart of the test interval. 
a. One-out-of-three (1/3) System. For a $1 / 3$ system, the unavailability of the system at any time in the test interval is given by

$$
\begin{aligned}
& 0<s \leq \tau_{t}, U(s)=(A+a T)(C+D \lambda T)^{2}, \\
& \tau_{t}<s \leq 2 \tau_{t}, U(s)=(B+b T)(A+a T)(C+D \lambda T), \\
& 2 \tau_{t}<s \leq 3 \tau_{t}, U(s)=(B+b T)^{2}(A+a T), \\
& 3 \tau_{t}<s \leq \theta, U(s)=(B+b T)^{3}, \\
& A<s \leq \theta+\tau_{t}, U(s)=(C+D \lambda \theta)(B+b T)^{2}, \\
& A+\tau_{t}<s \leq \theta+2 \tau_{t}, U(s)=(C+D \lambda \theta)^{2}(B+b T),
\end{aligned}
$$

and

$$
\theta+2 \tau_{t}<s \leq T, U(s)=(C+D \lambda s)^{3} \text {. }
$$

Again the assumption is made that if any component is in test or repair, the system unavailability is constant. The average unavailability for this system is:

\begin{tabular}{|c|c|c|c|}
\hline n & $\mathrm{T}^{\mathrm{n}}$ & $\left(\theta+2 \tau_{t}\right)^{n}$ & $\begin{array}{l}\text { ¿ercent } \\
\text { Errora }\end{array}$ \\
\hline 1 & 720 & 21 & 3 \\
\hline 2 & $5.184 \times 10^{5}$ & $4.41 \times 10^{2}$ & $1.51 \times 10^{-2}$ \\
\hline 3 & $3.71 \times 10^{\prime \prime}$ & $9.261 \times 10^{3}$ & $2.48 \times 10^{-3}$ \\
\hline 4 & $2.64 \times 10^{11}$ & $1.44 \times 10^{5}$ & $7.24 \times 10^{-5}$ \\
\hline 5 & $1.93 \times 10^{14}$ & $4.08 \times 10^{6}$ & $2.11 \times 10^{-6}$ \\
\hline
\end{tabular}

$$
\begin{aligned}
\overline{\mathrm{U}}_{1 / 3}= & (1 / T)\left\{\tau_{t}\left[A B^{2}+A B C+A C^{2}+B^{2} C+B C^{2}-5 C^{3}+B D \lambda \theta(B+2 C+D \lambda \theta)\right]\right. \\
& \left.+\nu\left(B^{3}-C^{3}\right)\right\}+\tau_{t}\left[A D \lambda(B+2 C)+A b(C+2 B)+a\left(B^{2}+B C+C^{2}\right)\right. \\
& +b(C+D \lambda \theta)(2 B+C+D \lambda \theta)]+3 \nu B^{2} b+C^{3}+T\left\{\tau_{t}[a D \lambda(B+2 C)\right. \\
& \left.\left.+A D \lambda(b+D \lambda)+a b(2 B+C)+b^{2}(A+C+D \lambda \theta)\right]+3 \nu B b^{2}+(3 / 2) C^{2} D \lambda\right\} \\
& +T^{2}\left\{\tau_{t}\left[a D \lambda(b+D \lambda)+a b^{2}\right]+\nu b^{3}+C D^{2} \lambda^{2}\right\}+(1 / 4) D^{3} \lambda^{3} T^{3}
\end{aligned}
$$

where $\nu=\theta-3 T_{t}$.

Again the integration of the last unavailability equation, Eq. 91, was performed over the interval 0 to $\mathrm{T}$ for terms other than $\mathrm{C}^{3}$. The errors are smaller than those shown in Table VIII. 
b. Two-out-of-three (2/3) System. For a $2 / 3$ system, the unavailability equations are

$$
\begin{array}{rl}
0<s & s \tau_{t}, U(s)=2(A+a T)(C+D \lambda T)+(C+D \lambda T)^{2} \\
& -2(A+a T)(C+D \lambda T)^{2}, \\
\tau_{t}<s \leq 2 \tau_{t}, U(s)=(A+a T)(B+b T)+(A+a T)(C+D \lambda T) & \\
& +(B+b T)(C+D \lambda T)-2(A+a T)(B+b T)(C+D \lambda T), \\
2 \tau_{t}<s \leq 3 \tau_{t}, U(s)=2(A+a T)(B+b T)+(B+b T)^{2} & <2(A+a T)(B+b T)^{2}, \\
3 \tau_{t}<s \leq 6, U(s)=3(B+b T)^{2}-2(B+b T)^{3}, \\
\theta<s & \leq \theta+T_{t}, U(s)=(B+b T)^{2}+2(B+b T)(C+D \lambda \theta) \\
& -(B+b T)^{2}(C+D \lambda \theta), \\
\theta+ & <s \leq \theta+2 \tau_{t}, U(s)=(C+D \lambda \theta)^{2}+2(B+b T)(C+D \lambda \theta) \\
& -2(B+b T)(C+D \lambda \theta)^{2},
\end{array}
$$

and

$$
\theta+2 \tau_{t}<s \leq T, U(s)=3(C+D \lambda s)^{2}-2(C+D \lambda s)^{3} .
$$

The average unavailability for this system is

$$
\begin{aligned}
\bar{U}_{2 / 3}= & (1 / T)\left\{T _ { t } \left[(1-2 A)\left(C^{2}+B C\right)+3 A C+A B(3-2 B)+2 B^{2}+2 B(C+D \lambda \theta)\right.\right. \\
& \left.\left.\cdot(2-B)+(C+D \lambda S)^{2}(1-2 B)-5 C^{2}(3-2 C)\right]+v B^{2}(3-2 B)-v C^{2}(3-2 C)\right\} \\
& +T_{t}\{(1-2 C)(2 A D \lambda+A b+a B)+2 C[D \lambda+a(1-C)]+D \lambda(A+B-2 A B) \\
& +C(a+b)+2 b(1-2 b)\left(A+C+D \lambda^{a}\right)+2 a B(1-B)+4 B b+2 b(C+D \lambda \theta) \\
& \cdot(1-C-D \lambda \theta)\}+6 \nu B b(1-B)+C^{2}(3-2 C)+T\left(T_{t}\{(1-2 C)(2 a D \lambda+a b)\right. \\
& \left.+(1-2 A)\left(b^{2}+b D \lambda+D^{2} \lambda^{2}\right)+(1-2 B)(a D \lambda+2 a b)+b^{2}[1-2(C+D \lambda \theta)]\right\} \\
& \left.+3 v b^{2}(1-2 B)+3 C D \lambda(1-C)\right)-T^{2}\left[2 a{ }^{\top} t\left(b^{2}+b D \lambda+D^{2} \lambda^{2}\right)+2 \nu b^{3}\right. \\
& \left.-D^{2} \lambda^{2}(1-2 C)\right]-(1 / 2) D^{3} \lambda^{3} T^{3} .
\end{aligned}
$$


c. Three-out-of-three (3/3) System. For a $3 / 3$ system, the unavailability at any time is given by:

$$
\begin{aligned}
& 0<s \leq \tau_{t}, U(s)=1-[1-(A+a T)][1-(C+D \lambda T)]^{2}, \\
& \tau_{t}<s \leq 2 \tau_{t}, U(s)=1-[1-(A+a T)][1-(B+b T)] \\
& \cdot[1-(C+D \lambda T)] \\
& 2 \tau_{t}<s \leq 3 \tau_{t}, U(s)=1-[1-(A+a T)][1-(B+b T)]^{2}, \\
& 3 \tau_{\mathrm{t}}<\mathrm{s} \leq \theta, \mathrm{U}(\mathrm{s})=1-[1-(\mathrm{B}+\mathrm{bT})]^{3} \text {, } \\
& \theta<s \leq \theta+\tau_{t}, U(s)=1-[1-(B+b T)]^{2}[1-(C+D \lambda \theta)], \\
& \theta+\tau_{t}<s \leq \theta+2 \tau_{t}, U(s)=1-[1-(B+b T)][1-(C+D \lambda \theta)]^{2},
\end{aligned}
$$

and

$$
\theta+2 \tau_{t}<s \leq T, U(s)=1-[1-(C+D \lambda s)]^{3}
$$

These equations give rise to the average unavailability equation

$$
\begin{aligned}
\bar{U}_{3 / 3}= & (1 / T)\left\{5 \tau_{t}(1-C)^{3}+\tau_{t}\left[(A-1)(C-1)(B+C-2)+(A-1)(B-1)^{2}\right.\right. \\
& \left.+(B-1)(1-C-D \lambda \theta)(2-B-C-D \lambda \theta)]+(1-C)^{3} \nu+(B-1)^{3} \nu\right\} \\
& +\tau_{t}\{(C-1)[2(A-1) D \lambda+a(C-1)]+(A-1)(B-1) D \lambda+b(A-1) \\
& +(2 B+C-3)+a(B-1)(B+C-2)+b(1-C-D \lambda \theta)(3-2 B-C-D \lambda \theta)\} \\
& +3(B-1)^{2} \nu b+1+(C-1)^{3}+T\left\{\tau_{t}[a(C-1)(b+2 D \lambda)+(A-1)\right. \\
& \left.\cdot\left(b^{2}+b D \lambda+D^{2} \lambda^{2}\right)+a(B-1)(2 b+D \lambda)-b^{2}(1-C-D \lambda \theta)\right]+3(B-1) \nu b^{2} \\
& \left.+(3 / 2) D \lambda(C-1)^{2}\right\}+T^{2}\left[a \tau_{t}\left(b^{2}+b D \lambda+D^{2} \lambda^{2}\right)+\nu D^{3}+(C-1) D^{2} \lambda^{2}\right] \\
& +(1 / 4) D^{3} \lambda^{3} T^{3} .
\end{aligned}
$$

\section{Four-component Systems}

The average unavailability equations for the $1 / 4,2 / 4,3 / 4$, and $4 / 4$ systems are pr. sented in the following sections.

a. One-out-of-four (1/4) System. The unavailability of a $1 / 4$ sys tem at any time $s$ is given by

$$
0<s \leq \tau_{t}, U(s)=(A+a T)(C+D \lambda T)^{3},
$$




$$
\begin{aligned}
& \tau_{t}<s \leq 2 \tau_{t}, U(s)=(A+a T)(B+b T)(C+D \lambda T)^{2}, \\
& 2 \tau_{t}<s \leq 3 \tau_{t}, U(s)=(A+a T)(B+b T)^{2}(C+D \lambda T) \\
& 3 \tau_{t}<s \leq 4 \tau_{t}, U(s)=(A+a T)(B+b T)^{3}, \\
& 4 \tau_{t}<s \leq \theta, U(s)=(B+b T)^{4} \\
& \theta<s \leq e+\tau_{t}, U(s)=(B+b T)^{3}(C+D \lambda \theta), \\
& \theta+\tau_{t}<s \leq G+2 \tau_{t}, U(s)=(B+b T)^{2}(C+D \lambda \theta)^{2}, \\
& \theta+2 \tau_{t}<s \leq \theta+3 \tau_{t}, U(s)=(B+b T)(C+D \lambda \theta)^{3},
\end{aligned}
$$

and

$$
\theta+3 \tau_{t}<s \leq T, U(s)=(C+D \lambda s)^{4}
$$

Interrating and taking the average yields

$$
\begin{aligned}
\overline{\mathrm{U}}_{1 / 4}= & (1 / T)\left(\tau _ { t } \left\{A\left(B^{3}+\mathrm{C}^{3}\right)+A B C(B+C)-7 C^{4}+B(C+D i \cdot)\left[B^{2}+B(C+D \lambda \theta)\right.\right.\right. \\
& \left.\left.\left.+(C+D \lambda \theta)^{2}\right]\right\}+\sigma\left(B^{4}-C^{4}\right)\right)+\tau_{t}\left\{(A D \lambda+a C)\left(B^{2}+B C+C^{2}\right)\right. \\
& +A C D \lambda(B+2 C)+A b C(2 B+C)+B^{2}[3 A b+a B+3 b(C+D \lambda \theta)] \\
& \left.+b(C+D \lambda f)^{2}(2 B+C+D \lambda \theta)\right\}+4 \sigma B^{3} b+C^{4}+T\left\{\tau_{t}[(A D \lambda+a C)\right. \\
& +(3 C D \lambda+B D \lambda+b C+B b)+(a B+A b)(C D \lambda+B D \lambda+b C+3 B b) \\
& \left.\left.+b^{2}(C+D \lambda G)(3 B+C+D \lambda \theta)\right]+6 \sigma B^{2} b^{2}+2 C^{3} D \lambda\right\}+T^{2}(\tau t\{(b+D \lambda) \\
& \left.\cdot[D \lambda(A D \lambda+2 a C)+b(2 a B+A b)]+a(B+C)\left(b^{2}+D^{2} \lambda^{2}\right)+b^{3}(C+D \lambda \theta)\right\} \\
& \left.+4 \sigma B b^{3}+2 C^{2} D^{2} \lambda^{2}\right)+T^{3}\left[a \tau t(b+D \lambda)\left(b^{2}+D^{2} \lambda^{2}\right)+\sigma b^{4}+C D^{3} \lambda^{3}\right] \\
& +(1 / 5) D^{4} \lambda^{4} T^{4},
\end{aligned}
$$

where $\sigma=6-4 \tau_{t}$.

The integration of Eq. 117 for terms other than $\mathrm{C}^{4}$ was done over the entire test interval. The subsequent errors are smaller than those listed in Table IX. 


\begin{tabular}{|c|c|c|c|}
\hline $\mathrm{n}$ & $T^{n}$ & $\left(\theta+3 T_{t}\right)^{n}$ & $\begin{array}{l}\text { Percent } \\
\text { Error }\end{array}$ \\
\hline$i$ & 720 & 23 & 3.30 \\
\hline 2 & $5.184 \times 10^{5}$ & $5.29 \times 10^{2}$ & $.02 \times 10^{-1}$ \\
\hline 3 & $3.73 \times 10^{8}$ & $1.22 \times 10^{4}$ & $3.26 \times 10^{-3}$ \\
\hline 4 & $2.69 \times 10^{11}$ & $2.80 \times 10^{5}$ & $1.04 \times 10^{-4}$ \\
\hline 5 & $1.93 \times 10^{14}$ & $6.34 \times 10^{6}$ & $3.33 \times 10^{-6}$ \\
\hline
\end{tabular}

b. Two-out-of-four (2/4) System. The unavailability equations for a $2 / 4$ system are

$$
\begin{aligned}
& 0<s \leq \tau_{t}, U(s)=3(A+a T)(C+D \lambda T)^{2}+(C+D \lambda T)^{3}-3(A+a T) \\
& (\mathrm{C}+\mathrm{D} \lambda \mathrm{T})^{3} \\
& \tau_{t}<s \leq 2 \tau_{t}, U(s)=2(A+a T)(B+b T)(C+D \lambda T)+(A+a T)(C+D \lambda T)^{2} \\
& +(B+b T)(C+D \lambda T)^{2}-3(A+a T)(B+b T)(C+D \lambda T)^{2}, \\
& 2 \tau_{t}<s \leq 3 \tau_{t}, U(s)=2(A+a T)(B+b T)(C+D \lambda T)+(A+a T)(B+b T)^{2} \\
& +(B+b T)^{2}(C+D \lambda T)-3(A+a T)(B+b T)^{2}(C+D \lambda T) \\
& 3 \tau_{t}<s \leq 4 \tau_{t}, U(s)=3(A+a T)(B+b T)^{2}+(B+b T)^{3}-3(A+a T) \\
& \cdot(B+b T)^{3}, \\
& 4 \tau_{t}<s \leq \theta, U(s)=4(B+b T)^{3}-3(B+b T)^{4}, \\
& \theta<s \leq \theta+\tau_{t}, U(s)=3(B+b T)^{2}(C+D \lambda \theta)+(B+b T)^{3}-3(B+b T)^{3} \\
& \cdot(C+D \lambda \theta) \\
& \theta+T_{t}<s \leq \theta+2 \tau_{t}, U(s)=2(B+b T)^{2}(C+D \lambda \theta)+2(B+b T)(C+D \lambda \theta)^{2} \\
& -3(B+b T)^{2}(C+D \lambda \theta)^{2} \\
& \left.\theta+2 \tau_{t}<s \leq \theta+3 \tau_{t}, U(s)=3(B+b T)(C+D \lambda \theta)^{2}+{ }_{1} C+D \lambda \theta\right)^{3} \\
& -3(\mathrm{~B}+\mathrm{bT})(\mathrm{C}+\mathrm{D} \lambda \theta)^{3}
\end{aligned}
$$


and

$$
\theta+3 \tau_{t}<s \leq T, U(s)=4(C+D \lambda s)^{3}-3(C+D \lambda s)^{4}
$$

The average unavailability for a $2 / 4$ system is

$$
\begin{aligned}
& \overline{\mathrm{U}}_{2 / 4}=(1 / T)\left(T _ { t } \left\{3 A C^{2}(1-C)+C^{3}+A B C(2-3 C)+C^{2}(A+B)+B^{2}(A+C)\right.\right. \\
& +B(2-3 B)\left[A C+(C+D \lambda \theta)^{2}\right]+3 B^{2}(1-B)(A+C+D \lambda \theta)+2 B^{3} \\
& \left.-7 C^{3}(4-3 C)+2(C+D \lambda \theta) B^{2}+3 B(C+D \lambda \theta)^{2}+(1-3 B)(C+D \lambda \theta)^{3}\right\} \\
& \left.+\sigma B^{3}(4-3 B)-\sigma C^{3}(4-3 C)\right)+\tau_{t}\{C(2-3 C)(3 A D \lambda+A b+a B) \\
& +3 C^{2}[a(1-C)+D \lambda]+2 A B D \lambda(1-3 C)+C[2 D \lambda(A+B)+C(a+b)] \\
& +(2-3 B) B[A D \lambda+a C+3 b(A+C+D \lambda \theta)]+2 b(1-3 B)\left[A C+(C+D \lambda \theta)^{2}\right] \\
& +B^{2}(a+D \lambda)+2 B b(A+C)+3 a B^{2}(1-B)+6 B^{2} b+4 B b(C+D \lambda \theta) \\
& \left.+3 b(C+D \lambda \theta)^{2}[1-(C+D \lambda \theta)]\right\}+12 \sigma B^{2} b(1-B)+C^{3}(4-3 C) \\
& +T\left(\tau_{t}\{D \lambda(1-3 C)[3 A D \lambda+2(A b+a B)]+a C(2-3 C)(b+3 D \lambda)\right. \\
& +D^{2} \lambda^{2}[A+B+3(C-A B)]+2(a+b) C D \lambda+b(1-3 B)[2(A D \lambda+a C) \\
& +3 b(A+C+D \lambda A)]+a B(2-3 B)(3 b+D \lambda)+2 B b(a+D \lambda)+b^{2}[1+C \\
& \left.+3(2 B-A C)]+b^{2}(C+D \lambda \theta)[2-3(C+D \lambda \theta)]\right\}+6 \sigma B b^{2}(2-3 B) \\
& \left.+6 C^{2} D \lambda(1-C)\right)+T^{2}\left(T _ { t } \left\{a(1-3 C)\left[b^{2}+D \lambda(2 b+3 D \lambda)\right]+(1-3 A)\right.\right. \\
& \cdot\left(D^{3} \lambda^{3}+b^{2} D \lambda+b^{3}\right)+D^{2} \lambda^{2}[a+b-3(A b+a B)]+a b(1-3 B)(3 b+2 D \lambda) \\
& \left.\left.+b^{3}[1-3(C+D \lambda \theta)]\right\}+4 \sigma b^{3}(1-3 B)+2 C D^{2} \lambda^{2}(2-3 C)\right)-T^{3}\left[3 a \tau_{t}(b+D \lambda)\right. \\
& \left.\cdot\left(b^{2}+D^{2} \lambda^{2}\right)+3 \sigma b^{4}-D^{3} \lambda^{3}(1-3 C)\right]-(3 / 5) D^{4} \lambda^{4} T^{4} .
\end{aligned}
$$

c. Three-out-of-four (3/4) System. The unavailability at any time $s$ for a $3 / 4$ system is given by

$$
\begin{aligned}
& 0<s \leq{ }_{t}, U(s)=3(A+a T)(C+D \lambda T)+3(C+D \lambda T)^{2}-6(A+a T)(C+D \lambda T)^{2} \\
&- 2(C+D \lambda T)^{3}+3(A+a T)(C+D \lambda T)^{3} \\
& T_{t}<s \leq 2 T_{t}, U(s)=(A+a T)(B+b T)+2(A+a T)(C+D \lambda T)+2(B+b T) \\
& \cdot(C+D \lambda T)+(C+D \lambda T)^{3}-4(A+a T)(B+b T)(C+D \lambda T)-2(A+a T) \\
& \cdot(C+D \lambda T)^{2}-2(B+b T)(C+D \lambda T)^{2}+3(A+a T)(B+b T)(C+D \lambda T),
\end{aligned}
$$




$$
\begin{aligned}
& 2 \tau_{t}<s \leq 3 \tau_{t}, U(s)=2(A+a T)(B+b T)+(f+a T)(C+D \lambda T)+2(B+b T) \\
& \cdot(C+D \lambda T)+(B+b T)^{2}-2(A+a T)(B+b T)^{2}-2(B+b T)^{2}(C+D \lambda T) \\
& -4(A+a T)(B+b T)(C+D \lambda T)+3(A+a T)(B+b T)^{2}(C+D \lambda T), \\
& 3 \tau_{t}<s \leq 4 \tau_{t}, U(s)=3(A+a T)(B+b T)+3(B+b T)^{2}-6(A+a T)(B+b T)^{2} \\
& -2(B+b T)^{3}+3(A+a T)(B+b T)^{3} \\
& 4 \tau_{t}<s \leq \theta, U(s)=6(B+b T)^{2}-8(B+b T)^{3}+3(B+b T)^{4}, \\
& \theta<s \leq \theta+\tau_{t}, U(s)=3(C+D \lambda \theta)(B+b T)+3(B+b T)^{2}-6(C+D \lambda \theta) \\
& (B+b T)^{2}-2(B+b T)^{3}+3(C+D \lambda \theta)(B+b T)^{3} \text {, } \\
& \theta+\tau_{t}<s \leq \theta+2 \tau_{t}, U(s)=(B+b T)^{2}+(C+D \lambda \theta)^{2}+4(B+b T)(C+D \lambda \theta) \\
& -4(B+b T)^{2}(C+D \lambda \theta)-4(B+b T)(C+D \lambda \theta)^{2} \\
& +3(B+b T)^{2}(C+D \lambda \theta)^{2} \\
& \theta+2 \tau_{t}<s \leq \theta+3 \tau_{t}, U(s)=3(C+D \lambda \theta)(B+b T)+3(C+D \lambda \theta)^{2}-6(B+b T) \\
& \text { - }(C+D \lambda \theta)^{2}-2(C+D \lambda \theta)^{3}+3(C+D \lambda \theta)^{3}(B+b T) \text {. }
\end{aligned}
$$

and

$\theta+3 \tau_{t}<s \leq T, U(s)=6(C+D \lambda s)^{2}-8(C+D \lambda s)^{3}+3(C+D \lambda s)^{4}$

Integrating the se equations gives rise to the average unavailability equation:

$$
\begin{aligned}
& \bar{r}_{3: 4}=(1 / T)\left(\tau _ { t } \left\{3 A C(C-1)^{2}+2 C^{2}(2-C)+A B C[3(B+C)-8]-7 C^{2}\left(6-8 C+3 C^{2}\right)\right.\right. \\
& +(A+B)\left(B+3 C-2 C^{2}\right)+B(A+C)(1-2 B)+B^{3}(3 A-?)+B^{2}[7-2 B \\
& -6(C+D \lambda \theta+A)]+4 A B+3 B(C+D \lambda \theta)\left[1+B^{2}+(C+D \lambda C-1)^{2}\right]+2(C+D \lambda A)^{2} \\
& \cdot[2-(C+D \lambda \theta)]+B(C+D \lambda \theta)[3 B(C+D \lambda \theta)-4(C+D \lambda \theta+B-1)]\} \\
& \left.+\sigma B^{2}\left(3 B^{2}-8 B+6\right)--C^{2}\left(3 C^{2}-8 C+6\right)\right)+\tau_{t}\left\{3 a C(C-1)^{2}+(3 C-1)(C-1)\right. \\
& \cdot(3 A D \lambda+A b+a B)-2 C(C-1)(a+b+3 D \lambda)+2 D \lambda(A(1-2 B)+B(1-2 C) \\
& +C(1-2 A) j+(A b+a 13)\left[3 B^{2}+3+C(3 B-4)\right]+b(A+C)(1-4 B) \\
& +B D \lambda(6 A C-4 A+1)+(A+B) D \lambda+(a+b)\left(2 B+C-6 B^{2}\right)-2 B^{2}(a+D \lambda) \\
& +3 A B(B D \lambda+b C)+b\left(A+2 B+6 A B^{2}\right)-6 B b[2(C+D \lambda G-1+A)+B]+b(C+D \lambda G) \\
& \left.\cdot\left[9 B^{2}+7-8 B+3(C+D \lambda A-1)^{2}\right]+2 b(C+D \lambda A)^{2}(3 B-2)\right]+12 B b \sigma(B-1)^{2} \\
& +C^{2}\left(3 C^{2}-8 C+6\right)+T\left[T _ { t } \left(3 a D \lambda(3 C-1)(C-1)+D^{2} \lambda^{2}(3 A B-2(A+B)-6 C+4]\right.\right.
\end{aligned}
$$




$$
\begin{aligned}
& +(3 C-2) D \lambda[2(A b+a B)+3 A D \lambda]+a b C(3 C-8)+a b\left(9 B^{2}+1\right)+b(1-4 B)(4 a+D \lambda) \\
& +(A b+a B)[3(B D \lambda+b C)-4 D \lambda]+(a+b)(b+3 D \lambda-4 C D \lambda)+b^{2}(3-8 A-2 C) \\
& +3 B b[\lambda D \lambda+a C+b(3 A-2)]+b^{2}\{3[(C+D \lambda \theta)(3 B-2)-2 B+1]+(C+D \lambda \theta-1) \\
& \left.+[3(C+D \lambda \theta)-1]\})+6 \sigma b^{2}(3 B-1)(B-1)+6 C D \lambda(C-1)^{2}\right]+T^{2}\left(\tau_{t}\{(3 A-2)\right. \\
& +\left(b^{3}+D^{3} \lambda^{3}\right)+a D \lambda(3 C-2)(2 b+3 D \lambda)+3 D \lambda(a B+A b)(b+D \lambda)-2 D^{2} \lambda^{2}(a+b) \\
& \left.+a b(3(B D \lambda+b C)-4 D \lambda]+b^{2}(9 a B-8 a-2 D \lambda)+b^{3}[3(C+D \lambda \theta)-2]\right\}+4 \sigma b^{3}(3 B-2) \\
& \left.+2 D^{2} \lambda^{2}(3 C-1)(C-1)\right)+T^{3}\left[3 a\left(b^{2}+D^{2} \lambda^{2}\right)(b+D \lambda) \tau_{t}+3 \sigma b^{4}+D^{3} \lambda^{3}(3 C-2)\right] \\
& +(3 / 5) D^{4} \lambda^{4} T^{4} .
\end{aligned}
$$

d. Four-out-of-four (4/4) System. Finally, for a $4 / 4$ system, the unavailability equations are

$$
\begin{aligned}
& 0<s \leq \tau_{t}, U(s)=1-[1-(A+a T)][1-(C+D \lambda T)]^{3}, \\
& \because<s \leq 2 T_{t}, U(s)=1-[1-(A+a T)][1-(B+b T)] \\
& \cdot[1-(C+D \lambda T)]^{2} \\
& 2 \tau_{t}\left\ulcorner s \leq 3 T_{t}, U(s)=1-[1-(A+a T)][1-(B+b T)]^{2}\right. \\
& \cdot[1-(C+D \lambda T)] \\
& 3 \tau_{t}<s \leq 4 \tau_{t}, U(s)=1-[1-(A+a T)][1-(B+b T)]^{3}, \\
& 4 T_{t}<s \leq \theta, U(s)=1-[1-(B+b T)]^{4}, \\
& \theta<s \leq \theta+\tau_{t}, U(s)=1-[1-(B+b T)]^{3}[1-(C+D \lambda \theta)] \\
& \theta+T_{t}<s \leq \theta+2 \tau_{t}, U(s)=1-[1-(B+b T)]^{2}[1-(C+D \lambda \theta)]^{2}, \\
& \theta+2 \tau_{t}<s \leq \theta+3 \tau_{t}, U(s)=1-[1-(B+b T)] \\
& \cdot[1-(C+D \lambda \theta)]^{3}
\end{aligned}
$$

and

$$
\theta+3 \tau_{t}<\mathrm{s} \leq \mathrm{T}, \mathrm{U}(\mathrm{s})=1-[1-(\mathrm{C}+\mathrm{D} \lambda \mathrm{s})]^{4}
$$

The average unavailability for this system is 


$$
\begin{aligned}
& \bar{U}_{4 / 4}=(1 / T)\left(7 \tau_{t}(1-C)^{4}-\tau_{t}\left\{(A-1)(C-1)^{3}+(B-1)\left[(A-1)(C-1)^{2}\right.\right.\right. \\
& \left.+(C+D \lambda \theta-1)^{3}\right]+(B-1)^{2}\left[(A-1)(C-1)+(C+D \lambda \theta-1)^{2}\right]+(B-1)^{3} \\
& \left.\cdot(\mathrm{A}+\mathrm{C}+\mathrm{D} \lambda \theta-2)\}+(\mathrm{C}-1)^{4} \sigma-(\mathrm{B}-1)^{4} \sigma\right)+1-T_{\mathrm{t}}\left\{(\mathrm{C}-1)^{2}[(\mathrm{~A}-1)\right. \\
& \cdot(b+3 D \lambda)+a(B+C-2)]+(A-1)(B-1)[b(C-1)+D \lambda(B+1)] \\
& +(B-1)[a(B-1)+b(A-1)](B+C-2)+b(B-1)^{2}[2(A-1) \\
& \left.+3(C+D \lambda \theta-1)]+b(C+D \lambda \theta-1)^{2}(2 B+C+D \lambda \theta-3)\right\}-4(B-1)^{3} \sigma b \\
& -(C-1)^{4}-T\left(r_{t}\{[(A-1) D \lambda+a(C-1)][3(C-1) D \lambda+b(B-1)]\right. \\
& +[a(B-1)+b(A-1)][(C-1)(b+2 D \lambda)+(B-1)(3 b+D \lambda)]+(A-1) \\
& \left.\cdot(B-1) D^{2} \lambda^{2}+a b(C-1)^{2}+(C+D \lambda \theta-1) b^{2}(3 P+C+D \lambda \theta-4)\right\} \\
& \left.+6(B-1)^{2} b^{2} \sigma+2(C-1)^{3} D \lambda\right)-T^{2}\left(\tau_{t}\{[a(B-1)+b(A-1)]\right. \\
& \cdot\left(b^{2}+b D \lambda+D^{2} \lambda^{2}\right)+(C-1) a\left(b^{2}+2 b D \lambda+3 D^{2} \lambda^{2}\right)+D \lambda[a b(B-1) \\
& \left.\left.+(A-1) D^{2} \lambda^{2}\right]+2 a(B-1) b^{2}+b^{3}(C+D \lambda \theta-1)\right\}+4(B-1) b^{3} \sigma \\
& \left.+2(C-1)^{2} D^{2} \lambda^{2}\right)-T^{3}\left[a \tau t\left(b^{2}+D^{2} \lambda^{2}\right)(b+D \lambda)+\sigma b^{4}+(C-1) D^{3} \lambda^{3}\right] \\
& -(1 / 5) D^{4} \lambda^{4} T^{4}
\end{aligned}
$$

\begin{tabular}{|c|c|c|c|c|c|}
\hline System & $\begin{array}{c}\text { Uniformly } \\
\text { Slaggered } \\
\text { Testing }\end{array}$ & $\begin{array}{l}\text { Nearly } \\
\text { Simultaneous } \\
\text { Testing }\end{array}$ & Syslem & $\begin{array}{c}\text { Unilormity } \\
\text { Staggeres } \\
\text { lesting }\end{array}$ & $\begin{array}{c}\text { Nearly } \\
\text { Simultaneous } \\
\text { Tesilng }\end{array}$ \\
\hline $1 / 2$ & 32 & 78 & $1 / 4$ & 57 & 118 \\
\hline $2 / 2$ & 37 & 84 & $2 / 4$ & 62 & 128 \\
\hline$B / 3$ & 42 & 92 & $3 / 4$ & 67 & 138 \\
\hline $2 / 3$ & 47 & 100 & $\$ / 4$ & 12 & 148 \\
\hline $3 B$ & 52 & 108 & & & \\
\hline
\end{tabular}

E. Calculation of Optimum Test Interval

Each of the average unavailability equations for the uniformly staggered and nearly simultancous testing schemes presented in the previous sections were of the form

$$
\overline{\mathrm{U}}(\mathrm{T})=\alpha / \mathrm{T}+\sum_{\mathrm{i}=0}^{\mathrm{n}} \beta_{\mathrm{i}} \mathrm{T}^{\mathrm{i}},
$$

where $n=$ number of redundancies in the system, and the coefficients $\alpha$ and $\beta_{i}$ are given by the appropriate equations in the preceding sections depending upon the type of system and testing scheme (see Table X). 
Taking $d \bar{U}(T) / d T$ and setting it equal to zero yields an equation of the form

$$
\sum_{i=1}^{n} i \beta_{i} T^{i+1}-\alpha=0
$$

To solve this equation, we use the Newton-Raphson method' for finding the roots of an equation. This method works in the following manner. An initial guess is made at the optimum test interval. (In this case, $T_{0}$ from Eq. 25 is sufficient since it is usually within a factor of 1.5 from the true optimum.) A new estimate is obtained from

$$
T_{n+1}=T_{n}-\frac{f\left(T_{n}\right)}{f^{\prime}\left(T_{n}\right)}
$$

where $f\left(T_{n}\right)$ is the value of Eq. 150 evaluated at $T_{n}$, and $f^{\prime}\left(T_{n}\right)$ is the derivative of Eq. 150 with respect to $T$ and evaluated $a \hat{t} T_{n}$.

If the convergence crierion is met $\left(T_{n+1}-T_{n}\right.$ less than some specified value), the process is halted. If not, the new guess, $T_{n+1}$, is substituted for $\mathrm{T}_{\mathrm{n}}$ on the right-hand side of Eq. 151.

F. Calculation of Fractional Unavailabilities

Occasionally, it becomes desirable to know how the contributions to the average unavailability break down among testing, repair, and random failures.

Consider a system having an average unavailability of $\vec{U}$ and the input test interval $T$. li the fraction of time the component is down during testing, $q_{0}$, is set equal to zero, a new value for the average unavailability, $\bar{U}_{N T}$, is obtained. The fraction of the average unavailability due to testing, $f_{l}$, is then

$$
f_{1}=\frac{\overline{\mathrm{U}}-\overline{\mathrm{U}}_{\mathrm{NT}}}{\overline{\mathrm{U}}}=1-\frac{\overline{\mathrm{U}}_{\mathrm{NT}}}{\overline{\mathrm{U}}} \text {. }
$$

To find the contribution made from repair, the parameters $B$ and $b$ from Eq. 19 are set equal to zero while maintaining $q_{0}$ at zero. This average unavailability $\bar{U}_{N T N R}$ is used to find the repair contribution, $f_{2}$, using the formula

$$
f_{2}=\frac{\bar{U}-\bar{U}_{N T N R}-\left(\bar{U}-\bar{U}_{N T}\right)}{\bar{U}}=\frac{\bar{U}_{N T}-\bar{U}_{N T N R}}{\bar{U}} .
$$


The fractional unavailability due to random failure is simply what remains, or

$$
f_{3}=1.0-f_{1}-f_{2} \text {. }
$$

These results can be used to determine whether testing and repair procedures should be modified to increase the reliability of the system.

\section{G. Summary}

The average unavailability equations for all $\mathrm{m} / \mathrm{n}(\mathrm{l} \leq \mathrm{m} \leq \mathrm{n} \leq 4)$ systems for random, uniformly staggered, and nearly simultaneous testing schemes have been developed. The equations for the uniformly staggered and nearly simultaneous testing schemes are listed in Table X. These equations, along with the methods for calculating the optimum test interval and fractional unavalabilities, have been incorporated into a computer code ICARUS. A user's manual for ICARUS is found in Appendix B. 


\section{COMPARISON OF ICARUS AND FRANTIC}

This chapter presents various sets of numerical comparisons between ICAR US and FRANTIC to determine the validity of the methodology and accuracy of the coding found in ICARUS. Also, multicomponent redundancies are treated.

\section{A. Introduction}

FRANTIC is the acronym for the Formal Reliability Analysis including Normal Testing, Inspection, and Checking computer code developed at the NRC. It calculates time-dependent and average unavailabilities, and also the breakdown of the unavailability contributions from testing, repair, and random failures.

FRANTIC is abl- to treat four kinds of components: constantunavailability components, non repairable components, monitored components, and periodically tested components. The main emphasis in this study is to obtain the average unavailability of periodically tested components given such information as the test duration time, repair time, test-override capability, and test efficiency.

B. Differences between ICARUS and FRANTIC

1. Testing Srhemes

In ccmparing ICARUS and FRANTIC, we must recognize that FRAN'TIC is more general than ICARUS. ICARUS was developed to treat only periodically tested components, while FRANTIC is able to handle a variety of components. Also, FRANTIC calculates time-depender.t and average unavailabilities for any test interval (not necessarily in the asynuptotic state). Since this option is available, the user can choose the length of the first test interval for each component and thus stagger the test intervals for the components in whatcver manner is desired. ICARUS, on the other hand, calculates the average unavailability only in the asymptotic state. As a consequence, with ICARUS, the user must choose one of the three available testing schemes rather than creating a particular testing scheme through the input, as with FRANTIC.

\section{Numerical Calculations}

In regard to numerical calculations, FRANTIC calculates the average unavailability by multiplying the unavailability of each component at specified times, and performing trapezoidal-rule integration. Consequently, F'RANTIC cannot calculate the optimum test interval internally, whereas ICARUS, which is totally analytical in its treatment of the average unavailability equations, calculates the optimum test interval by direct differentiation. 
Also, the analytical treatment in ICARUS avoids any inherent numerical error. FRANTIC, for example, can give errors up to a factor of 2.5 for quadruply redundant systems.

\section{Component Types}

FRANTIC can treat systems made lip of different types of component, whereas ICARUS assumes the redundancies in the system to be identical. A formulation has been developed to treat multicomponent redundancies in ICARUS and is presented in Scc. IV.D below

\section{Failure Modes}

Finally, ICARUS is able to treat threc failure modes not found in FRANTIC:

$Y_{r}$, failure to start due to true demand,

$f_{f}$, failure to detect or repair a failure,

and

$\rho_{u}$, failure due to a test demand that is not repaired.

Since the purpose of this research is to incorporate as many failure modes as possible and to evaluate their importance, a morc in-clepth study of these failure modes is performed in Chapter $V$.

C. Comparison of ICARUS and FRANTIC Simple Systems

To verify the coding and accuracy of the average unavailability equations found in ICARUS, we used ICARUS and FRANTIC to calculate the average unavailabilities of systems made up of one valve per redundancy. This was done for both staggered and nearly simultaneous testing schemes using only the failure modes common to both codes.

\section{Comparison of Uniformly Staggered Testing Scheme}

The comparison data for a single valve are given in Table XI. Table XII shows the average unavailability value obtained by the two codes and the percent difference hetween them. All the differences are less than $2.0 \%$ with the majority less than $1.0 \%$. Since this kind of study usually requires order-of-magnitude accuracy, the differences here are well within acceptable bounds.

\section{Comparison of Nearly Simultaneous Testing Scheme}

The data in Table XI were also applied to the nearly simultaneous testing scheme. Nole that $\tau_{t}=3.0 \mathrm{~h}$ and $\tau_{r}=10.0 \mathrm{~h}$; therefore, $\tau_{r}$ is always greater than $(n-1) \tau_{t}$ for all $n \leq 4$. Table Xlll gives the results of the comparisons. Again, all the differences are small $(\leq 1.1 \%)$. 
TABLE XI. Input Data for Single-valve Redundancy

\begin{tabular}{ll}
\hline Failure rate $(\lambda)$ & $3.0 \times 10^{-7} / \mathrm{h}$ \\
Fraction of downtime during test $\left(q_{0}\right)$ & $1.0 \times 10^{-1}$ \\
Probability of failure due to test demand $\left(Y_{e}\right)$ & $1.0 \times 10^{-3} / \mathrm{d}$ \\
Test period duration $\left(\tau_{t}\right)$ & $3.0 \mathrm{~h}$ \\
Repair period duration $(\tau)$ & $10.0 \mathrm{~h}$ \\
Test interval $(T)$ & $720 \mathrm{~h}$ \\
\hline
\end{tabular}

TABLE XIl. Comparison of ICARUS and FRANTIC Results for Uniformly Staggered Testing

\begin{tabular}{cccc}
\hline System & \multicolumn{2}{c}{$\bar{U}$} & $\begin{array}{c}\text { Percent } \\
\text { Difference }\end{array}$ \\
\cline { 2 - 4 } $1 / 4$ & $1.73 \times 10^{-15}$ & $1.73 \times 10^{-15}$ & 0 \\
$1 / 3$ & $1.45 \times 10^{-11}$ & $1.46 \times 10^{-11}$ & 0.68 \\
$2 / 4$ & $6.01 \times 10^{-11}$ & $6.00 \times 10^{-11}$ & 0.17 \\
$1 / 2$ & $1.04 \times 10^{-7}$ & $1.06 \times 10^{-7}$ & 1.88 \\
$2 / 3$ & $3.16 \times 10^{-7}$ & $3.16 \times 10^{-11}$ & 0 \\
$3 / 4$ & $6.34 \times 10^{-1}$ & $6.31 \times 10^{-7}$ & 0.48 \\
$1 / 1$ & $5.46 \times 10^{-4}$ & $5.45 \times 10^{-4}$ & 0.18 \\
$2 / 2$ & $1.10 \times 10^{-3}$ & $1.09 \times 10^{-3}$ & 0.92 \\
$3 / 3$ & $1.65 \times 10^{-1}$ & $1.64 \times 10^{-3}$ & 0.61 \\
$4 / 4$ & $2.21 \times 10^{-3}$ & $2.18 \times 10^{-3}$ & 1.38 \\
\hline
\end{tabular}

$\frac{\left|\overline{\mathrm{U}}_{F}-\overline{\mathrm{U}}_{\mathrm{I}}\right|}{\overline{\mathrm{U}}_{\mathrm{F}}}$ 
TABLE XIII. Comparison of ICARUS and FRANTIC Results for Nearly Simultaneous Testing

\begin{tabular}{|c|c|c|c|}
\hline \multirow[b]{2}{*}{ System } & \multicolumn{2}{|c|}{$\overline{\mathrm{U}}$} & \multirow{2}{*}{$\begin{array}{c}\text { Percent } \\
\text { Difference }\end{array}$} \\
\hline & ICARUS & FRANTIC & \\
\hline $1 / 4$ & $9.24 \times 10^{-13}$ & $9.24 \times 10^{-13}$ & 0 \\
\hline $1 / 3$ & $7.66 \times 10^{-10}$ & $7.67 \times 10^{-10}$ & 0.13 \\
\hline $2 / 4$ & $305 \times 10^{-9}$ & $3.05 \times 10^{-9}$ & 0 \\
\hline $1 / 2$ & $6.33 \times 10^{-7}$ & $6.40 \times 10^{-7}$ & 1.09 \\
\hline $2 / 3$ & $1.89 \times 10^{-6}$ & $1.91 \times 10^{-6}$ & 1.05 \\
\hline $3 / 4$ & $3.77 \times 10^{-6}$ & $3.81 \times 10^{-6}$ & 1.05 \\
\hline $1 / 1$ & $5.46 \times 10^{-4}$ & $5.45 \times 10^{-i}$ & 0.18 \\
\hline $2 / 2$ & $1.09 \times 10^{-3}$ & $1.09 \times 10^{-1}$ & 0 \\
\hline $3 / 3$ & $1.64 \times 10^{-1}$ & $1.63 \times 10^{-3}$ & 0.61 \\
\hline $4 / 4$ & $2.18 \times 10^{-3}$ & $2.18 \times 10^{-1}$ & 0 \\
\hline
\end{tabular}

Note that the unavailabilities for the uniformly staggered testing scheme werc lower than the nearly simultaneous testing scheme in all cases, except for the $n / n$ systems, where the average unavailabilities were calculated to be virtually indentical.

D. Treatment of Multicomponent Redundancies

As stated before, the ICARUS code was written to handle systems made up of single-component redundancies. However, we can represent a multicomponent redundancy by an effective "single" component and thus apply ICARUS to the more complicated multicomponent systems if each of the nine ICARUS input parameters are handled correctly. The method for handling each parameter is discussed in the following sections. The theoretical basis for the disfinitions can be found in Ref. 7 .

1. Test Interval, $T_{\text {eff }}$

Since the components in the redundancies are all assumed to have the same test interval $T$, it remains unchanged. Thus $T_{\text {eff }}=T$.

2. Test-period Duration, $T_{\text {teff }}$

Each component in the redundancy must be tested in the test period. Therefore, $T_{\text {teff }}$ will be the sum of the individual $\tau_{t}$ 's for the components in the redundancy. That is, 


$$
\tau_{\text {teff }}=\sum_{j=1}^{k} \tau_{t j}
$$

where $k$ equals the number of components in the redundancy.

\section{Repair-period Duration, $\tau_{\text {reff }}$}

The repair-period duration, $\tau_{r}$, of the redundancy depends upon the probability of the components in the redundancy failing, and the time $r \in$ quired to repair a failed component. The probability that a component is repaired after a test (per test) is given by

$$
R=\hat{u}\left(l-\rho_{f}\right)+(1-\hat{u}) \gamma_{e}=\gamma_{e}+\frac{\rho_{u}+\lambda T}{1-\left(\rho_{f}-\rho_{u}\right)}\left[1-\left(\rho_{f}+\gamma_{e}\right)\right] \text {. }
$$

For the redundancy, the effective repair-period duration is

$$
\tau_{r}=\frac{\sum_{j=1}^{k} R_{j} r_{j}}{\sum_{j=1}^{k} R_{j}},
$$

where the repair times $\tau_{r j}$ of components $j$ are weighted by the $R_{j}$ values defined above for each component.

4. Fraction of Downtime during Testing, $q_{0 \text { eff }}$

The total downtime due to testing is assumed to be the sum of the downtimes of the components in series. Thus, the effective fractional downtime value is calculated from

$$
q_{0} \text { eff }=\frac{\sum_{j=1}^{k} \tau_{t j} q_{0 j}}{\tau_{t e f f}} .
$$

\section{Remainder of the Parameters}

The remainder of the parameters--the constant failure rate $(\lambda)$, failure to start on true demand $\left(Y_{r}\right)$, failure due to test demand (repaired, $Y_{e}$ not repaired, $\left.\rho_{u}\right)$, and failure to detect or repair a failure $\left(\rho_{u}\right)$--are treated the same way as the test-period duration. The values for the parameter in question for each component are added together to get an effective valuc for that parameter. 
E. ICARUS and FRANTiC Comparisons for Two-valves-per-redundancy Systems

ICARUS and FRANTIC values for the average unavailabilities were compared for a set of systems that contained two valves in series for each redundancy. For FRANTIC, the values of each parameter for a single valve are given in Table $\mathrm{XiV}$.

TABLE XIV. ICARUS and FRANTIC Input Values for Multicomponent Redundancies

\begin{tabular}{ccc}
\hline Paran.eter & $\begin{array}{c}\text { ICARUS } \\
\text { Value }\end{array}$ & $\begin{array}{c}\text { FRANTIC Value } \\
\text { (per Valve) }\end{array}$ \\
\hline$\gamma_{\mathrm{e}}$ & 0.002 & 0.001 \\
$\mathrm{q}_{0}$ & $6.0 \times 10^{-7} / \mathrm{h}$ & $3.0 \times 10^{-7} / \mathrm{h}$ \\
$\tau_{\mathrm{r}}$ & $1.0 \times 10^{-\mathrm{t}^{\mathrm{a}}}$ & $1.0 \times 10^{-1}$ \\
$\tau_{\mathrm{t}}$ & $10.0 \mathrm{~h}$ & $10.0 \mathrm{~h}$ \\
$\mathrm{~T}$ & $6.0 \mathrm{~h}^{\mathrm{a}}$ & $3.0 \mathrm{~h}$ \\
\hline
\end{tabular}

aICARUS will change these values internally if necessary, to ineet the input criteria.

With the methods of Secs. IV.D. I-IV.D.5, the values used in ICARUS for two valves in series (one redundancy) are also given in Table XIV.

1. Comparison of Uniformly Staggercd Testing Scheme

The average unavailabilities calculated by ICARUS asd FRANTIC for the two-valve-per-redundancy systems are listed in Table XV for the uniformly staggered testing scheme.

In each case, the difference is less than $3 \%$, indicating that the averaging scheme used by ICARUS to represent multicomponent redundancies is adequate.

2. Comparison of Nearly Simultaneous Testing Scheme

The average unavailabilities for the two-valve-per-redundancy systems tested nearly simultaneously are listed in Table XVI. Unlike the previous comparisons, large differences appear in five of the ten cases. It is not clear which of the codes is incorrect since fairly good agreement between the two was established in earlier comparisons. 
TABLE XV. Conparis on of ICARUS and FRANTIC Results for Two Valves per Redundancy and Uniformly Staggered Testing

\begin{tabular}{llcc}
\hline System & $\overline{\mathrm{U}}$ & FRANTIC & $\begin{array}{c}\text { Percent } \\
\text { Difference }\end{array}$ \\
\cline { 2 - 4 } $1 / 4$ & ICARUS & $2.741 \times 10^{-14}$ & 1.79 \\
$1 / 3$ & $2.790 \times 10^{-14}$ & $1.152 \times 10^{-10}$ & 1.48 \\
$2 / 4$ & $1.169 \times 10^{-10}$ & $4.771 \times 10^{-10}$ & 2.99 \\
$1 / 2$ & $4.852 \times 10^{-10}$ & $4.159 \times 10^{-7}$ & 1.70 \\
$2 / 3$ & $4.213 \times 10^{-7}$ & $1.255 \times 10^{-6}$ & 1.51 \\
$3 / 4$ & $1.274 \times 10^{-6}$ & $2.517 \times 10^{-6}$ & 1.79 \\
$1 / 1$ & $2.562 \times 10^{-6}$ & $1.090 \times 10^{-3}$ & 1.01 \\
$2 / 2$ & $1.101 \times 10^{-3}$ & $2.179 \times 10^{-3}$ & 1.51 \\
$3 / 3$ & $2.212 \times 10^{-3}$ & $3.268 \times 10^{-3}$ & 1.96 \\
$4 / 4$ & $3.332 \times 10^{-3}$ & $4.356 \times 10^{-3}$ & 0.09 \\
\hline 6 & $4.360 \times 10^{-3}$ & &
\end{tabular}

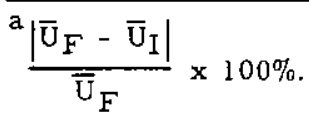

TABLE XVI. Comparison of ICARUS and FRANTIC Results for Two Valves per Redundancy and Nearly Simultaneous Testing

\begin{tabular}{cccc}
\hline System & ICARUS & $\overline{\mathrm{U}}$ & $\begin{array}{c}\text { Percent } \\
\text { Difference }\end{array}$ \\
\hline $1 / 4$ & $1.473 \times 10^{-11}$ & $1.357 \times 10^{-12}$ & 985.48 \\
$1 / 3$ & $6.087 \times 10^{-4}$ & $3.147 \times 10^{-9}$ & 93.42 \\
$2 / 4$ & $2.429 \times 10^{-8}$ & $7.913 \times 10^{-9}$ & 206.96 \\
$1 / 2$ & $2.534 \times 10^{-6}$ & $2.506 \times 10^{-6}$ & 1.12 \\
$2 / 3$ & $7.556 \times 10^{-6}$ & $6.294 \times 10^{-6}$ & 20.05 \\
$3 / 4$ & $1.504 \times 10^{-5}$ & $1.052 \times 10^{-5}$ & 42.97 \\
$1 / 1$ & $1.101 \times 10^{-3}$ & $1.090 \times 10^{-3}$ & 1.09 \\
$2 / 2$ & $2.204 \times 10^{-3}$ & $2.177 \times 10^{-3}$ & 1.24 \\
$3 / 3$ & $3.296 \times 10^{-3}$ & $3.263 \times 10^{-3}$ & 1.01 \\
$4 / 4$ & $4.360 \times 10^{-3}$ & $4.348 \times 10^{-3}$ & 0.28 \\
\hline
\end{tabular}

$\frac{\mathrm{a}\left|\overline{\mathrm{U}}_{F}-\overline{\mathrm{U}}_{\mathrm{I}}\right|}{\overline{\mathrm{U}}_{\mathrm{F}}} \times 100 \%$ 
One possible source of error is the numerical-integration scheme used by FRANTIC to calculate the average unavailability. FRANTIC chooses points on the time-dependent unavailability curve where changes in slope occur. Then, using trapezoidal-rule integration, we obtain the area under the curve. When applying this to nearly simultaneous testing, note that the changes in slope occur at the start of the interval during test and repair. The rest of the interval is dominated by random failure upon which the method may break down. To show this would require an exact calculation of the average unavailability of one of the systems in question.

\section{Exact Calculations for Average Unavailability of $1 / 3$ System}

The exact calculations for the average unavailability of a $1 / 3$ system with two valves per reduldincy were performed using the following equations, where for notational simplicity, $A_{0}=1-(A+a T)$ and $B_{0}=1-$ $(\mathrm{B}+\mathrm{bT})$ :

$$
\begin{aligned}
T- & 5 \tau_{t}<s \leq T-4 \tau_{t}, U(s)=\left\{1-A_{0}\left[1-\lambda\left(s+4 \tau_{t}\right)\right]\right\}\left\{1-\left[1-\lambda\left(s+3 \tau_{t}\right)\right]\right. \\
& \left.\cdot\left[1-\lambda\left(s+2 \tau_{t}\right)\right]\right\}\left\{1-\left[1-\lambda\left(s+\tau_{t}\right)\right](1-\lambda s)\right\}, \\
T- & 4 \tau_{t}<s \leq T-3 \tau_{t}, U(s)=\left(1-B_{0} A_{0}\right)\left\{1-\left[1-\lambda\left(s+3 \tau_{t}\right)\right]\left[1-\lambda\left(s+2 \tau_{t}\right)\right]\right\} \\
& \cdot\left\{1-\left[1-\lambda\left(s+\tau_{t}\right)\right](1-\lambda s)\right\}, \\
T- & 3 \tau_{t}<s \leq T-2 \tau_{t}, U(s)=\left(1-B_{0}^{2}\right)\left\{1-A_{\lambda}\left[1-\lambda\left(s+2 \tau_{t}\right)\right]\right\}\left\{1-\left[1-\lambda\left(s+\tau_{t}\right)\right]\right. \\
& \cdot(1-\lambda s)\}, \\
T- & 2 \tau_{t}<s s T-\tau_{t}, U(s)=\left(1-B_{0}^{2}\right)\left(1-B_{0} A_{i} !\left\{1-\left[1-\lambda\left(s+\tau_{t}\right)\right](1-\lambda s)\right\},\right.
\end{aligned}
$$$$
T-\tau_{t}<s \leq T, U(s)=\left(1-B_{0}^{2}\right)^{2}\left[1-A_{0}(1-\lambda s)\right]
$$$$
0<s s_{t}, U(s)=\left(1-B_{0}^{2}\right)^{2}\left(1-B_{0} A_{0}\right) \text {, }
$$$$
\tau_{t}<s \leq \tau_{r}-4 \tau_{t}, \quad U(s)=\left(1-B_{0}^{2}\right)^{3} \text {, }
$$

$$
\begin{aligned}
& \tau_{r}-4 \tau_{t}<s \leq \tau_{r}-3 \tau_{t}, U(s)=\left\{1-\left[1-\lambda\left(s+5 \tau_{t}\right)\right] B_{0}\right\}\left(1-B_{0}^{2}\right)^{2}, \\
& \tau_{t}-3 \tau_{t}<s s \tau_{r}-2 \tau_{t}, U(s)=\left\{1-\left[1-\lambda\left(s+5 \tau_{t}\right)\right]\left[1-\lambda\left(s+4 \tau_{t}\right)\right]\right\}\left(1-B_{0}^{2}\right)^{2},
\end{aligned}
$$

$$
\begin{aligned}
\tau_{r}- & 2 \tau_{t}<s \leq \tau_{r}-\tau_{t}, U(s)=\left\{1-\left[1-\lambda\left(s+5 \tau_{t}\right)\right]\left[1-\lambda\left(s+4 \tau_{t}\right)\right]\right\} \\
& \cdot\left\{1-\left[1-\dot{\lambda}\left(s+3 \tau_{t}\right)\right] B_{0}\right\}\left(1-B_{0}^{2}\right),
\end{aligned}
$$




$$
\begin{aligned}
{ }_{\tau_{r}}-\tau_{t}<s \leq \tau_{r}, U(s)=\left\{1-\left[1-\lambda\left(s+5 \tau_{t}\right)\right]\left[1-\lambda\left(s+4 \tau_{t}\right)\right]\right\}\left\{1-\left[1-\lambda\left(s+3 \tau_{t}\right)\right]\right. \\
\left.\cdot\left[1-\lambda\left(s+2 \tau_{t}\right)\right]\right\}\left(1-B_{0}^{2}\right) \\
\tau_{r}<s \leq \theta, U(s)=\left\{1-\left[1-\lambda\left(s+5 \tau_{t}\right)\right]\left[1-\lambda\left(s+4 \tau_{t}\right)\right]\right\}\left\{1-\left[1-\lambda\left(s+3 \tau_{t}\right)\right]\right. \\
\left.\cdot\left[1-\lambda\left(s+2 \tau_{t}\right)\right]\right\}\left\{1-\left[1-\lambda\left(s+\tau_{t}\right)\right] B_{0}\right\}
\end{aligned}
$$

and

$$
\begin{gathered}
\theta<s \leq T-5 \tau_{t}, U(s)=\left\{1-\left[1-\lambda\left(s+5 \tau_{t}\right)\right]\left[1-\lambda\left(s+4 \tau_{t}\right)\right]\right\}\left\{1-\left[1-\lambda\left(s+3 \tau_{t}\right)\right]\right. \\
\left.\cdot\left[1-\lambda\left(s+2 \tau_{t}\right)\right]\right\}\left\{1-\left[1-\lambda\left(s+\tau_{t}\right)\right](1-\lambda s)\right\} .
\end{gathered}
$$

These equations result from knowing that the unavailability of two valves in series is $1-\left(1-U_{1}\right)\left(1-U_{2}\right)$. Thus for three parallel sets of two valves in series, the unavailability is

$$
U_{1 / 3}=\left[1-\left(1-U_{1}\right)\left(1-U_{2}\right)\right]\left[1-\left(1-U_{3}\right)\left(1-U_{4}\right)\right]\left[1-\left(1-U_{5}\right)\left(1-U_{6}\right)\right] \text {. }
$$

Also note that for this set of equations, $\tau_{r}$ must be greater than $3 \tau_{t}$.

With the data in the first column of Table XVII for each valve, the average unavailability of the system was calculated to be $3.111 \times 10^{-9}$.

TABLE XVII. Parameter Values for Test System

\begin{tabular}{clll}
\hline Parameter & $\begin{array}{c}\text { Exact } \\
\text { Calculation }\end{array}$ & $\begin{array}{c}\text { ICARUS } \\
\text { Input }\end{array}$ & $\begin{array}{c}\text { FRANTIC } \\
\text { Input }\end{array}$ \\
\hline$\lambda$ & $3.0 \times 10^{-7} / \mathrm{h}$ & $6.0 \times 10^{-7} / \mathrm{h}$ & $3.0 \times 10^{-7} / \mathrm{h}$ \\
$\gamma_{\mathrm{e}}$ & $1.0 \times 10^{-3}$ & $2.0 \times 10^{-3}$ & $1.0 \times 10^{-3}$ \\
$\mathrm{q}_{0}$ & 0.10 & 0.10 & 0.10 \\
${ }_{\mathrm{T}} \mathrm{r}$ & $10.0 \mathrm{~h}$ & $10.0 \mathrm{~h}$ & $10.0 \mathrm{~h}$ \\
$\mathrm{~T}_{\mathrm{t}}$ & $1.5 \mathrm{~h}$ & $3.0 \mathrm{~h}$ & $1.5 \mathrm{~h}$ \\
$\mathrm{~T}$ & $720 \mathrm{~h}$ & $720 \mathrm{~h}$ & $720 \mathrm{~h}$ \\
\hline
\end{tabular}

With the data in the secolid column of Table XVII for each redundancy, the average unavailability for the same system was calculated using ICARUS. The value obtained for this method was $\bar{U}_{1 / 3}=3.145 \times 10^{-9}$. 
Finally, with the data from the third column of Table XVII, the dverage unavailability was calculated using the FRANTIC code. The value obtained was $2.310 \times 10^{-8}$, which is significantly different from the ICARUS value. To identify why the difference in values occurred, we made two more FRANTIC runs. In the first run, the $q_{0}$ value was halved and the $\tau_{t}$ value doubled to maintain the $q_{0} T_{t}$ value of testing unavailability. The error is small since, in the tosting period, the equations are

$$
\begin{aligned}
\operatorname{Exact} \bar{U} & =\left(\tau_{t} / T\right)\left[q_{0}+\left(1-q_{0}\right)\left(\gamma_{e}+\lambda T\right)\right] \\
= & \left(\tau_{t} / T\right)\left[q_{0}+\left(1-q_{0}\right) \gamma_{e}\right]+\left(1-q_{0}\right) \lambda \tau_{t} \\
\text { Approximate } \bar{U} & =\left(2 \tau_{t} / T\right)\left[q_{0} / 2+\left(1-q_{0} / 2\right)\left(\gamma_{e}+\lambda T\right)\right] \\
& =\left(\tau_{t} / T\right)\left[q_{0}+\left(2-q_{0}\right) \gamma_{e}\right]+\left(2-q_{0}\right) \lambda \tau_{t} .
\end{aligned}
$$

This leads to an error of about $1 \%$ in this case. The value obtained using these data is $\bar{U}_{1 / 3}=1.610 \times 10^{-9}$. This is closer to the ICARUS value.

Next, the original $q_{0}$ was multiplied by 2 and $\tau_{t}$ was halved. The value obtained for this case is $\overline{\mathrm{U}}_{1 / 3}=3.112 \times 10^{-9}$. This is nearly the exact answer calculated by analytical means.

F. Summary

It is not clear what problems arise in modeling nearly simultaneously tested systems using FRANTIC. The three cases presented above should give approximately the same answers, but instead differ by an order of magnitude in at least one of the cases.

As for ICARUS, the averaging methods used to simulate multicomponent redundancy systems appear adequate in light of previous verification runs. 


\section{ANALYSIS OF AN AUXILIARY FEEDWATER SYSTEM}

In this chapter, the ICARUS code is used to calculate the average unavailability of an auxiliary feedwater system for the three testing schemes. Also, the sensitivity of the unavailability values to the ICARUS input is determined.

\section{A. Description}

The auxiliary feedwater system (AFWS) provides feedwater to the secondary side of the main steam generator upon the los of main feedwater. Figure 10 is a simplified fiow diagram of the AFWS.

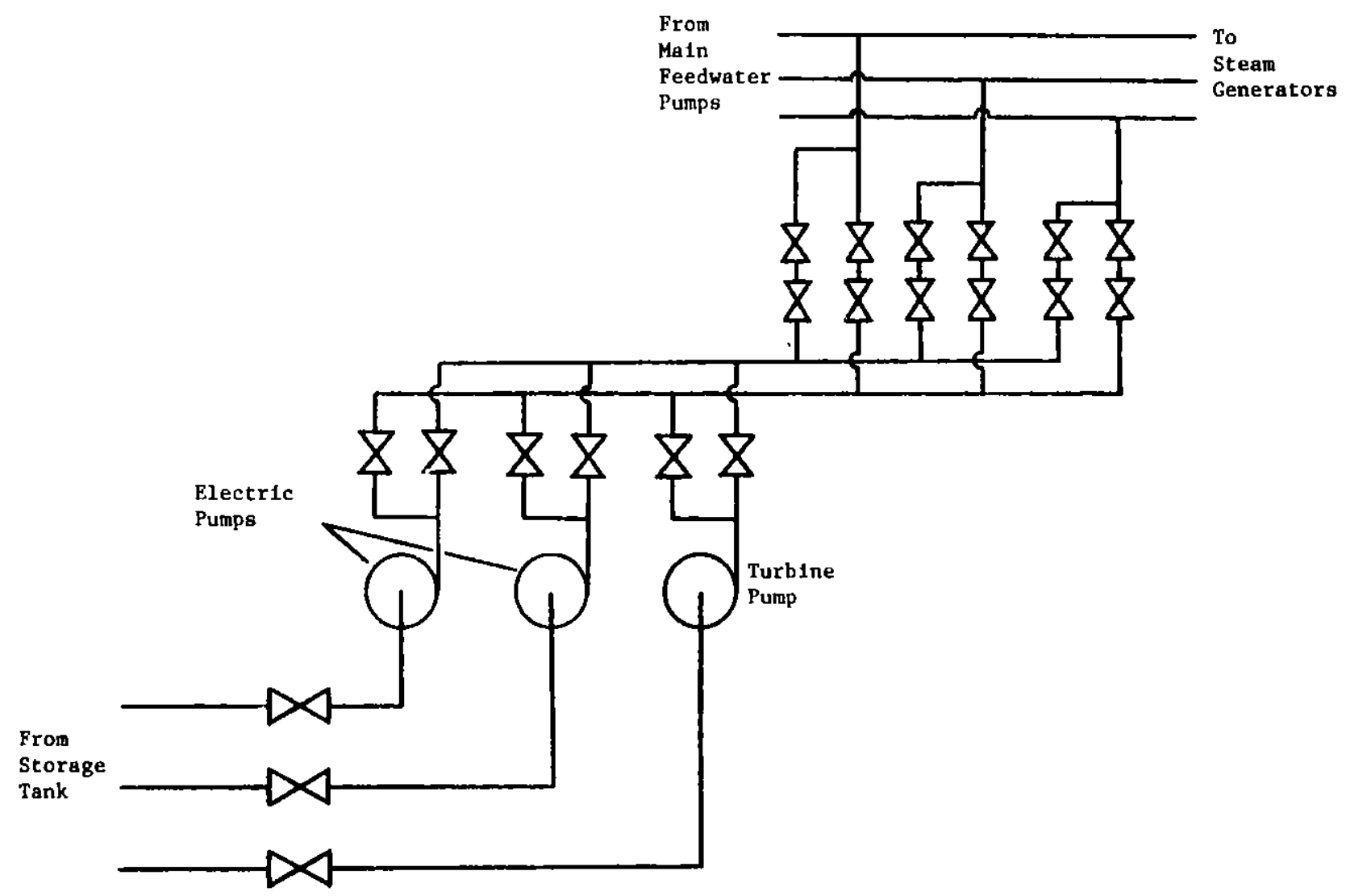

Fig. 10. Simplified Drawing of Auxiliary Feedwater System

The following paragraphs are taken from WASH-1400, ${ }^{10}$ and describe an auxiliary feedwater system for a typical PWR 3-loop design.

"Given a need for the AFWS, the three pumps, two electric $(350 \mathrm{gpm})$ and one turbine $(700 \mathrm{gpm})$ can be started either automatically or manually. The electric pumps are started automatically when: (1) a Safety Injection Control System signal is present; (2) loss of offsite power is detected; (3) main feedwater pumps shut off; or (4) low water level is detected by a steam generator. The turbine pump is started when low water level in a steam generator is detected or loss of offsite power is detected. 
"All pumps are aligned to the 110,000 gallon condensate tank via separate suction lines at all times, except when maintenance is being performed on a pump. The three pumps deliver water to two headers which penetrate containment. Inside containment, each steam generator can receive condensate from either header.

"All the decay heat produced can be removed by any one of three steam generators. The amount of feedwater decreases with time, and the operators can throttle flow to steam generators by shutting off $\mathrm{re}$ dundant pumps, and then utilizing the motor operated valves inside containment, decrease the flow as necessary to match the steam produced and released."

The pump test is performed by closing the valve(s) leading to the header, opening the valve(s) on a return loop to the $110,000-$ gal $\left(416-\mathrm{m}^{3}\right)$ reservoir, and then operating the pump. in this way, the pump is tested without clraining the auxiliary feedwater supply. During the test, the pump is not available since it is valved off from the secondary loop.

B. Data Base

Components such as pumps, valves, and Diesel generators are not exclusive to the nuclear industry. Because of this, a large data base is available for component parameters such as failure rates, test-period durations, and repair-period durations. Extensive data were analyzed and reported in WASH-1400 and will be used in this work whenever appropriate.

1. Values of $\rho_{u}$ and $\rho_{f}$

The data available on human error are small and not as easily found as data on equipment failures. In particular, there is no source of numbers from which to assign values to the failure modes $\rho_{u}$ (test fails component and is not repaired) and $\rho_{f}$ (test fails to detect a component as failed). Millan and Edison ${ }^{11}$ have tabulated a list of auxiliary-feedwater-system failures that occurred in 1974-1976, the plants at which they occurred, their causes, and the docket number for each occurrence. The list was studied, and possible candidates for the failure modes ( $\rho_{u}$ and $\rho_{f}$ ) were selected. The dockets were then read, and each selected failure was assigned a $\rho_{u}$ or $\rho_{f}$ designation. Case failures are summarized in Appendix F.

In all, there were six $\rho_{\mathrm{u}}$ failures and two $\rho_{f}$ failures. In the threeyear period, there were 3209 PWR reported occurrences, 95 of which were auxiliary-feedwater-system failures. During this period, also, the 35 power plants studied had accumulated about 83.3 years of operating experience. If we assume that each plant had an auxiliary feedwater system with three redundancies, and each redundancy was tested monthly, the total number of test demands on the components is about 3000. A value for $\rho_{u}$ can be calculated using

$$
p_{u}=6 \text { failures } / 3000 \text { demands }=2.0 \times 10^{-3} / \mathrm{d} \text {. }
$$


Calculating a number for $\rho_{f}$ is not quite as straightforward. A $\rho_{f}$ failure can only occur if there had been a previous failure of the system and an unsuccessful repair. The value of $\rho_{f}$ is thus calculated from

$$
\rho_{f}=2 \text { failures/95 AFWS failures }=2.11 \times 10^{-2} .
$$

Although the value of $\rho_{f}$ is greater than that of $\rho_{u}$, the contribution it makes to the average unavailability of the system is not as large, since the probability of an auxiliary-feedwater-system failure is in itself small.

\section{Values of $\gamma_{\mathrm{e}}$ and $\gamma_{\mathbf{r}}$}

Since the auxiliary-feedwater-system failure data were available, it was decided to calculate values for test and true-demand failures, $\gamma_{e}$ and $\gamma_{r}$, directly. Since, the only difference between $\gamma_{e}$ and $\gamma_{r}$ is the time at which the failure (initiating event) occurs, the total number of failures on clemand was used to calculate the values of both $\gamma_{a}$ and $\gamma_{r}$.

In Ref. 11, three instances of failures on demand were found among the 3000 demands. These are also given in Appendix F. To be on the conservative side, the value obtained for failure on demand was assumed to be valid for both $\gamma_{e}$ and $\gamma_{r}$, giving

$$
\gamma_{e}=\gamma_{r}=3 \text { failures } / 3000 \text { demands }=1.0 \times 10^{-3} / \mathrm{d}
$$

C. Numerical Analysis

Computer runs of ICARUS were made to calculate the average unavailability of the auxiliary feedwater system in three cases:

Case I: plant steam available, offsite power available.

Case II: no plant steam, offsite power available.

Case III: no plant steam, no offsite power.

1. Case I: Plant Steam Available, Offsite Power Available

Case I models the auxiliary feedwater system when steam is available to power the turbine pump and offsite power is present to operate the two electric pumps. The auxiliary feedwater system would be called upon if, for example, a low water level was detected in the steam generators. To simplify the system for an ICARUS calculation, the system was idealized to look like the one in Fig. 11. This is a $1 / 3$ system since only one pump is needed to supply the steam generators. Also, since these are multicomponent redundancies, the averaging equations presented in Chapter IV are us ed to model the system. 


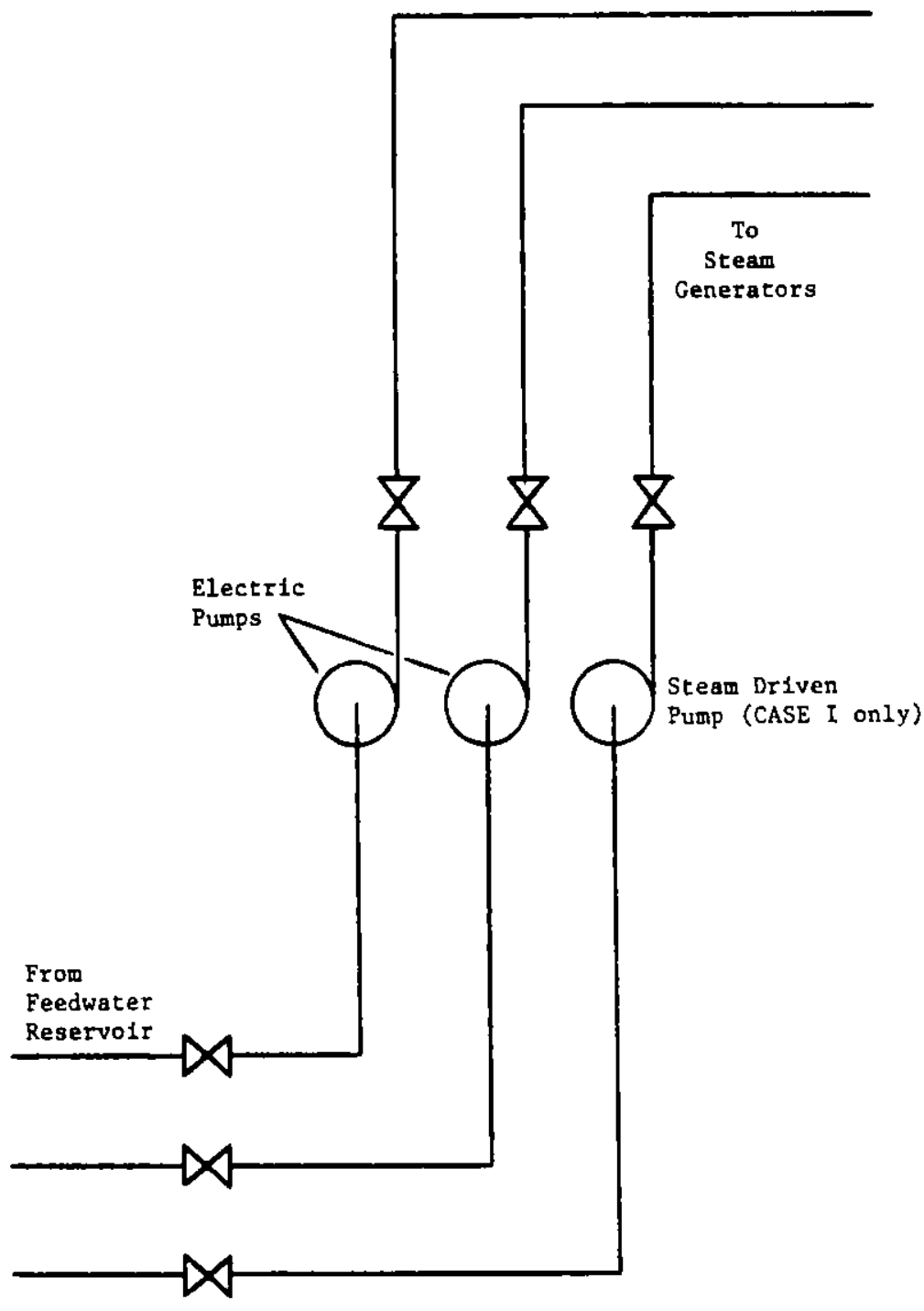

Fig. 11

systenı Drawing for Cases I and II

a. Effective Duration of Redundancy Test Period and Fraction of Downtime. The following values $0_{1}$ t were obtained from Ref. 10 , Table III 5-1:

$$
{ }^{\top} \mathrm{t}_{\text {Pump }}=1.4 \mathrm{~h},{ }^{\top} \mathrm{t}^{\mathrm{V}} \mathrm{Valve}=0.86 \mathrm{~h}
$$

However, since the pump and valves are tested at the same time, the test-period duration of the effective component redundancy is that of a pump plus some additional time to align the valves properly (approximately one valve-test duration time) or

$$
\tau_{\text {teff }}=2.26 \mathrm{~h} \text {. }
$$

As for $q_{0}$, all the components are taken offline when testing is done. Thus, the value of $q_{0}$ for the effective component redundancy is 1.0.

b. Effective Repair-period Duration of Redundancy. The effective repair-period duration of the redundancy is equal to the average of the repair times for each component weighted by the $R$ factor of Eq. 156 for each 
component (i.e., by the probability of having to repair the component). The following data. were obtained from Ref. 10, Table III 5-3:

$$
\tau_{\text {Valve }}=7.0 \mathrm{~h} \text { and } \tau_{\text {Purrsp }}=19.0 \mathrm{~h}
$$

The $\mathrm{R}$ term was derived previously as

$$
R=\gamma_{e}+\hat{u}\left(l-\rho_{f}-\gamma_{e}\right)=\gamma_{e}+\rho_{u}+\lambda T .
$$

The values of $Y_{e}, Y_{r}$, and $\rho_{u}$ obtained in Sec. V.B.2 were for a single redundancy rather than for individual components. Since three components make up each redundancy in Case $I$, the $\gamma_{e}, \gamma_{r}, \rho_{f}$, and $\rho_{u}$ values obtained in Sec. V.B. 2 are divided by 3 to obtain values for each component in the redundancy to be used to determine the repair-period duration. Thus, for the three components,

$$
\gamma_{e}=\gamma_{r}=3.33 \times 10^{-4}, \rho_{f}=7.018 \times 10^{-3} \text {, and } \rho_{u}=6.67 \times 10^{-4} \text {. }
$$

A value for the constant failure rate is also needed for each component, and a problem arises since the failure rates found in WASH-1400 are for components in operation rather than on standby. To obtain a standby $\lambda$, the probability of failure on demand, $Q_{d}$, for each component (Ref. 10, Table III 4-2) was divided by the length of one test interval to obtain a failure rate, since

$$
Q_{d}=\lambda T \text {. }
$$

For a valve, $Q_{d}=1.0 \times 10^{-4}$. For a test interval of $720 \mathrm{~h}$, $\lambda_{\mathrm{v}}=1.389 \times 10^{-7} / \mathrm{h}$. The $\mathrm{R}$ value for a valve equals

$$
R=3.33 \times 10^{-4}+6.67 \times 10^{-4}+1.0 \times 10^{-4}=1.1 \times 10^{-3} \text {. }
$$

For the pump, $Q_{\mathrm{dp}}=1.0 \times 10^{-3}$. For a test interval of $720 \mathrm{~h}, \lambda_{\mathrm{p}}=$ $1.389 \times 10^{-6} / \mathrm{h}$. The $\mathrm{R}$ value equals

$$
R=3.33 \times 10^{-4}+6.67 \times 10^{-4}+1.0 \times 10^{-3}=2.0 \times 10^{-3} \text {. }
$$

The value of $\tau \mathbf{r}$ for the effective component redundancy is calculated from

$$
{ }^{\tau} r_{\text {eff }}=\frac{2 R_{v^{\top}} \mathrm{rv}+R_{p^{\top}} r p}{2 R_{v}+R_{p}}=12.7 \mathrm{~h} .
$$

c. Redundancy Failure Rate. The failure rate for the redundancy can be approximated as the sum of the failure rates of the components in the redundancy:

$$
\lambda_{\text {eff }}=2 \lambda_{\mathrm{v}}+\lambda_{\mathrm{p}}=1.67 \times 10^{-6} / \mathrm{h} .
$$


d. ICARUS Results for Random Testing Scheme. The average unavailability, optimum test interval, and unavailability fractions due to testing, repair, and random failures were calculated for the system by ICARUS assuming completely random testing. The results are shown in Table XVIII. The major contributor to the average unavailability is testing, followed by random failures and, finally, repair. The large testing factor is due to the system being completely unavailable during the test-period duration $\left(q_{0}\right.$ eff $\left.=1.0\right)$.

TABLE XVIII. ICARUS Results for Case I

\begin{tabular}{|c|c|c|c|}
\hline $\begin{array}{l}\text { Testing } \\
\text { Scheme }\end{array}$ & $\begin{array}{l}\text { Completely } \\
\text { Random }\end{array}$ & $\begin{array}{l}\text { Uniformly } \\
\text { Staggered }\end{array}$ & $\begin{array}{c}\text { Nearly } \\
\text { Simultaneous }\end{array}$ \\
\hline $\begin{array}{l}\text { Average } \\
\text { Unavailability }\end{array}$ & $3.23 \times 10^{-7}$ & $1.77 \times 10^{-7}$ & $3.75 \times 10^{-7}$ \\
\hline $\begin{array}{l}\text { Optimum } \\
\text { Test } \\
\text { Interval, } \mathrm{h}\end{array}$ & 1624.4 & 1183.2 & 1381.3 \\
\hline $\begin{array}{l}\text { Average } \\
\text { Unavailability } \\
\text { at TOPTIMUM }\end{array}$ & $2.03 \times 10^{-7}$ & $1.62 \times 10^{-7}$ & $3.22 \times 10^{-7}$ \\
\hline $\begin{array}{l}\text { Unavailability } \\
\text { due to Testing }(\%)\end{array}$ & $\begin{array}{c}2.71 \times 10^{-7} \\
(83.7)\end{array}$ & $\begin{array}{c}1.25 \times 10^{-7} \\
(70.6)\end{array}$ & $\begin{array}{c}3.17 \times 10^{-7} \\
(84.7)\end{array}$ \\
\hline $\begin{array}{l}\text { Unavailability } \\
\text { due to Repair }(\%)\end{array}$ & $\begin{array}{c}2.90 \times 10^{-9} \\
(0.90)\end{array}$ & $\begin{array}{c}2.81 \times 10^{-9} \\
(1.6)\end{array}$ & $\begin{array}{c}6.56 \times 10^{-9} \\
(1.7)\end{array}$ \\
\hline $\begin{array}{l}\text { Unavailability } \\
\text { due to Random } \\
\text { Failures }(\%)\end{array}$ & $\begin{array}{c}4.97 \times 10^{-8} \\
(15.4)\end{array}$ & $\begin{array}{c}4.92 \times 10^{-8} \\
(27.8)\end{array}$ & $\begin{array}{c}5.10 \times 10^{-8} \\
(13.6)\end{array}$ \\
\hline
\end{tabular}

The optimum average test interval is 68 days, which is longer than the 30 -day standard. In general, this points to a large test and repair contribution and, as seen in Table XVIII, these two contributions make up about $85 \%$ of the average unavailability.

The average unavailability at the optimum test interval is a factor of 1.6 times lower than that at the 30-day interval. Average unavailability versus test interval is plotted in Fig. 12. The curve decreases rapidiy with time at short test intervals, but after reaching a minimum, increases rather slowly. 


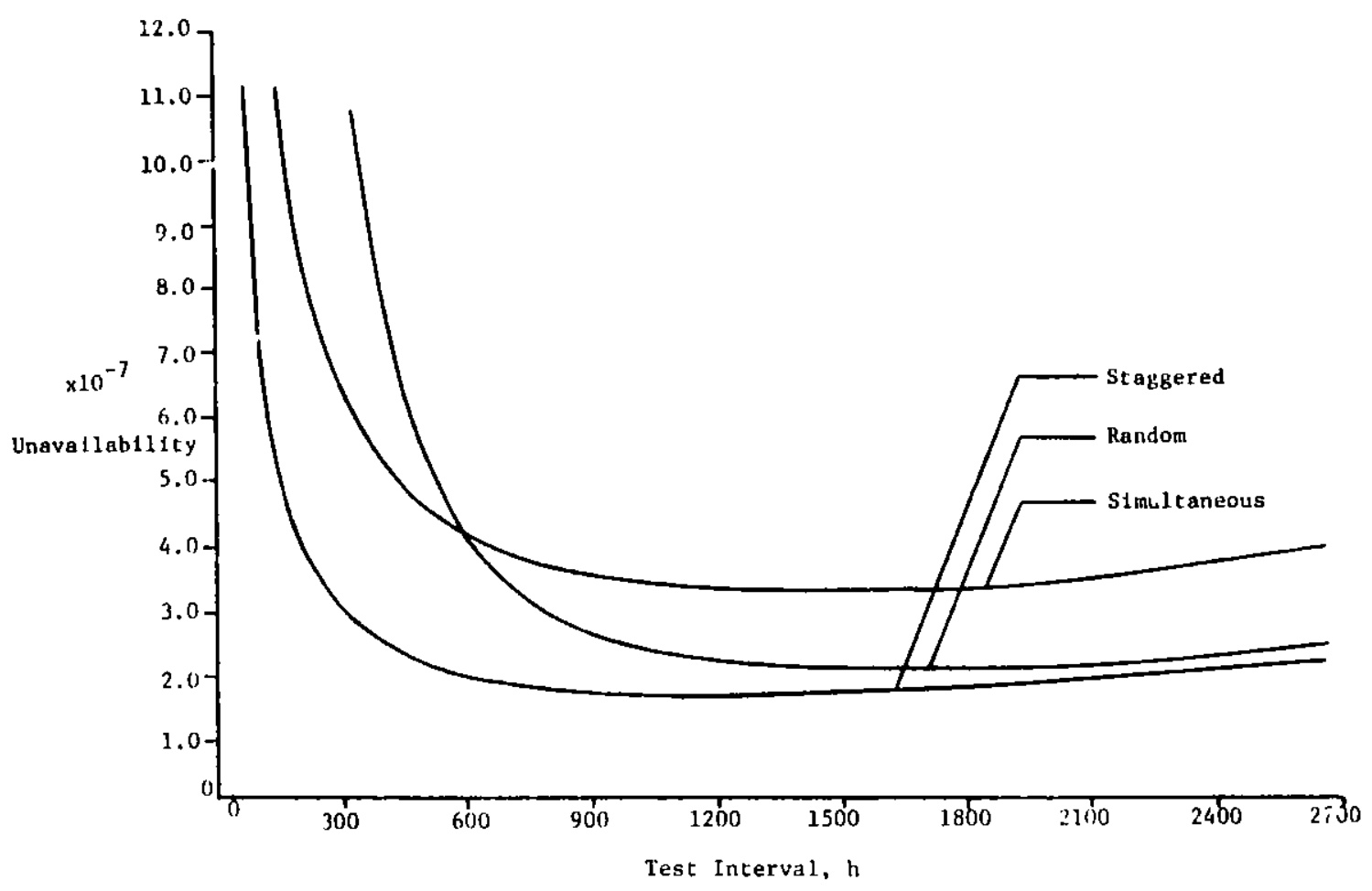

Fig. 12. Average Unavailability vs Test Interval for Case I

e. ICARUS Results for Uniformly Staggered Testing. The results of ICARUS runs made for the uniformly staggered testing scheme are also shown in Table XVIII. Again the testing of the system contributes the most $(70.6 \%)$ to the average unavailability. Also, the optimum test interval is about 49 days. This is longer than for the once-per-month test, but less than the optimum using random testing. The longer test interval is again due to the high test contribution to the average unavailability.

Average unavailability versus test interval is plotted in Fig. 12. The curve is of the same shape as the curve for the random testing scheme, except that the magnitude at each test interval is smaller.

f. ICARUS Results for Nearly Simultaneous Testing. The results for the nearly simultaneous testing scheme for Case I are also shown in Table XVIII. The average unavailability contribution due to testing is very high and makes the overall average unavailability the highest of all three testing schemes for test intervals greater than $600 \mathrm{~h}$. The optimum test interval is long (57.6 days) due to testing, and the unavailability at the optimum is the highest of all the schemes. Average unavailability versus test interval is plotted in Fig. 22.

2. Case II: Plant Steam Not Available, Offsite Power Available

Case II models a plant at a time when no steam is present to operate the turbine pump, but the electric pumps do have an outside power 
source. This system would look like the one in Fig. 11 with the turbine pump having an unavailability equal to 1.0 . This is a $1 / 2$ system having the same ICARUS input as Case 1 .

a. ICARUS Results for Random Testing Scheme. The results for the ICARUS run of the $1 / 2$ system are shown in Table XIX. The average unavailability for the random testing scheme is primarily due to testing $70.2 \%$ ), with random failure $(28.7 \%)$ and repair contributions $(1.1 \%)$ following in order. The average unavailability for this $1 / 2$ system is about 150 times greater than for the same scheme in Case I. The removal of one redundancy can thus greatly affect the performance of a system.

TABLE XIX. ICARUS Results for Case II

\begin{tabular}{|c|c|c|c|}
\hline $\begin{array}{l}\text { Testing } \\
\text { Scheme }\end{array}$ & $\begin{array}{l}\text { Completely } \\
\text { Random }\end{array}$ & $\begin{array}{l}\text { Uniformly } \\
\text { Staggered }\end{array}$ & $\begin{array}{c}\text { Nearly } \\
\text { Simultaneous }\end{array}$ \\
\hline $\begin{array}{l}\text { Average } \\
\text { Unavailability }\end{array}$ & $4.71 \times 10^{-5}$ & $3.68 \times 10^{-5}$ & $5.05 \times 10^{-5}$ \\
\hline $\begin{array}{l}\text { Optimum } \\
\text { Test } \\
\text { Interval, } h\end{array}$ & 1624.4 & 1403.1 & 1572.7 \\
\hline $\begin{array}{l}\text { Average } \\
\text { Unavailability } \\
\text { at TOPTIMUM }\end{array}$ & $3.45 \times 10^{-5}$ & $3.19 \times 10^{-5}$ & $4.13 \times 10^{-5}$ \\
\hline $\begin{array}{l}\text { Unavailability } \\
\text { due to Testing }(\%)\end{array}$ & $\begin{array}{c}3.31 \times 10^{-5} \\
(70.2)\end{array}$ & $\begin{array}{c}2.28 \times 10^{-5} \\
(62.1)\end{array}$ & $\begin{array}{c}3.59 \times 10^{-5} \\
(71.1)\end{array}$ \\
\hline $\begin{array}{l}\text { Unavailability } \\
\text { due to Repair }(\%)\end{array}$ & $\begin{array}{c}5.20 \times 10^{-7} \\
(1.1)\end{array}$ & $\begin{array}{c}5.13 \times 10^{-7} \\
(1.4)\end{array}$ & $\begin{array}{c}9.44 \times 10^{-7} \\
(1.9)\end{array}$ \\
\hline $\begin{array}{l}\text { Unavailability } \\
\text { due to Random } \\
\text { Failures }(\%)\end{array}$ & $\begin{array}{c}1.35 \times 10^{-5} \\
(28.7)\end{array}$ & $\begin{array}{c}1.35 \times 10^{-5} \\
(36.5)\end{array}$ & $\begin{array}{c}1.37 \times 10^{-5} \\
(27.0)\end{array}$ \\
\hline
\end{tabular}

The optimum test interval is again 68 days (see Sec. III.B), and the average unavailability at this interval is only a factor of 1.37 times less than for the once-per-month test. Average unavailability versus test interval is plotted in Fig. 13. The random scheme has very high unavailability for short test intervals because the probability of having both components of the system tested near or at the same time becomes more probable as the test interval shortens. 


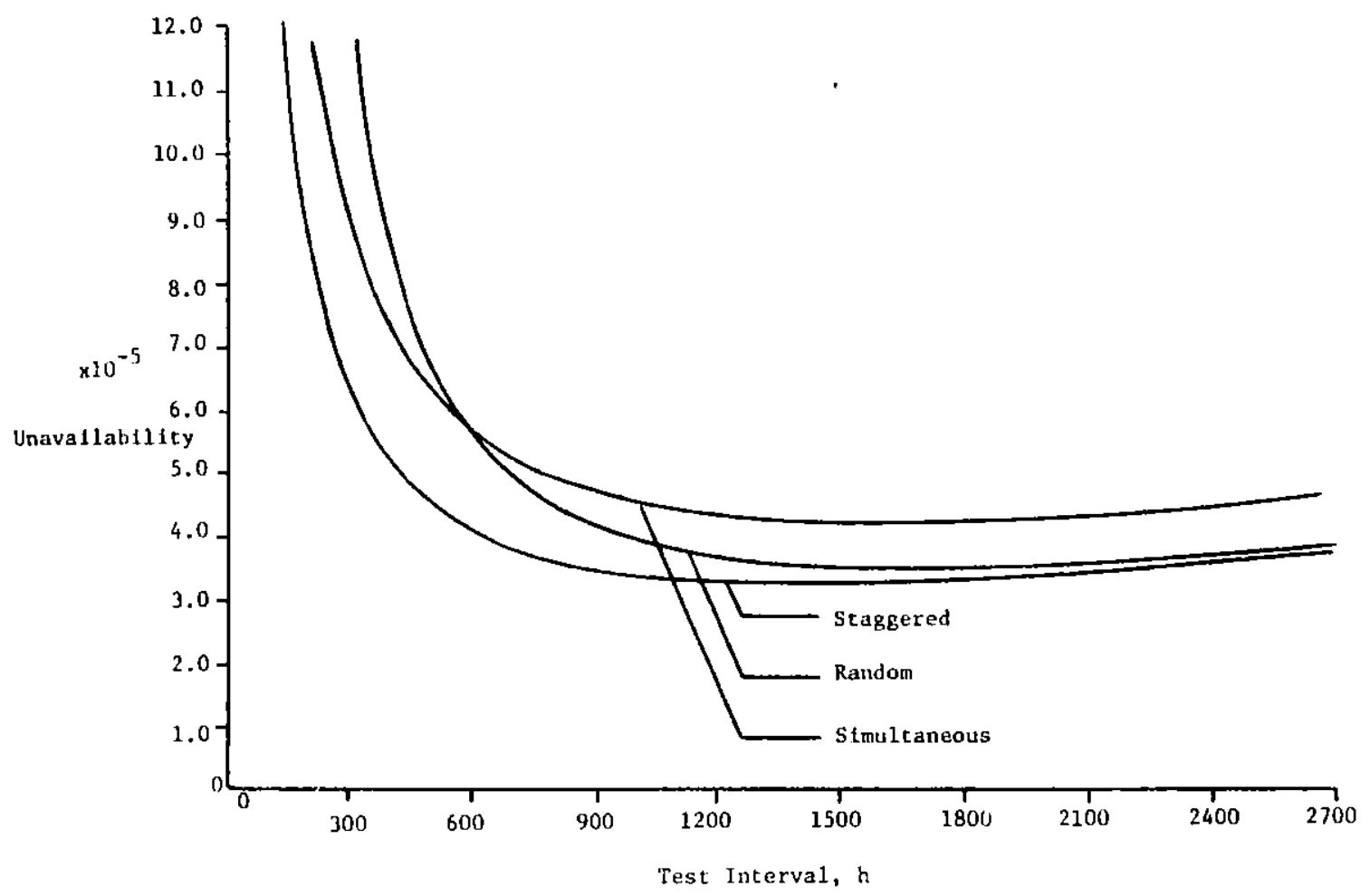

Fig. 13. Average Unavailability vs Test Interval for Cise II

b. ICARUS Results for Uniformly Staggered Testing. The results for the uniformly staggered testing scheme are listed in Table XIX. The average unavailability is again lower than for random testing, as is the average unavailability at the optimum test interval. The contribution to the unavailability is mostly from testing. The optimum test interval occurs at 58.8 days. Figure 13 is a plot of average unavailability versus test interval.

c. ICARUS Results for Nuarly Simultaneous Testing. Table XIX presents the results of the Case II analysis with nearly simultaneous testing. The average unavailability is again the highest of the three testing schemes for test intervals greater than $630 \mathrm{~h}$. Testing is the dominant factor in the unavailability, driving the optimum test interval up to 65.5 days. The average unavailability at the optimum is 1.22 times less than for once-per-month nearly simultaneous testing. Average unavailability versus test interval is plotted in Fig. 13.

\section{Case III: No Steam Available, No Offsite Power Available}

In Case III, both main power sources for the pumps are gone. There is no steam to drive the turbine pump, and the electric pumps must rely on Diesel generators to operate. The idealized system is shown in Fig. 14. Since all the components in each redundancy must operate, the series system ave:aging equations of Chapter IV must be used. 


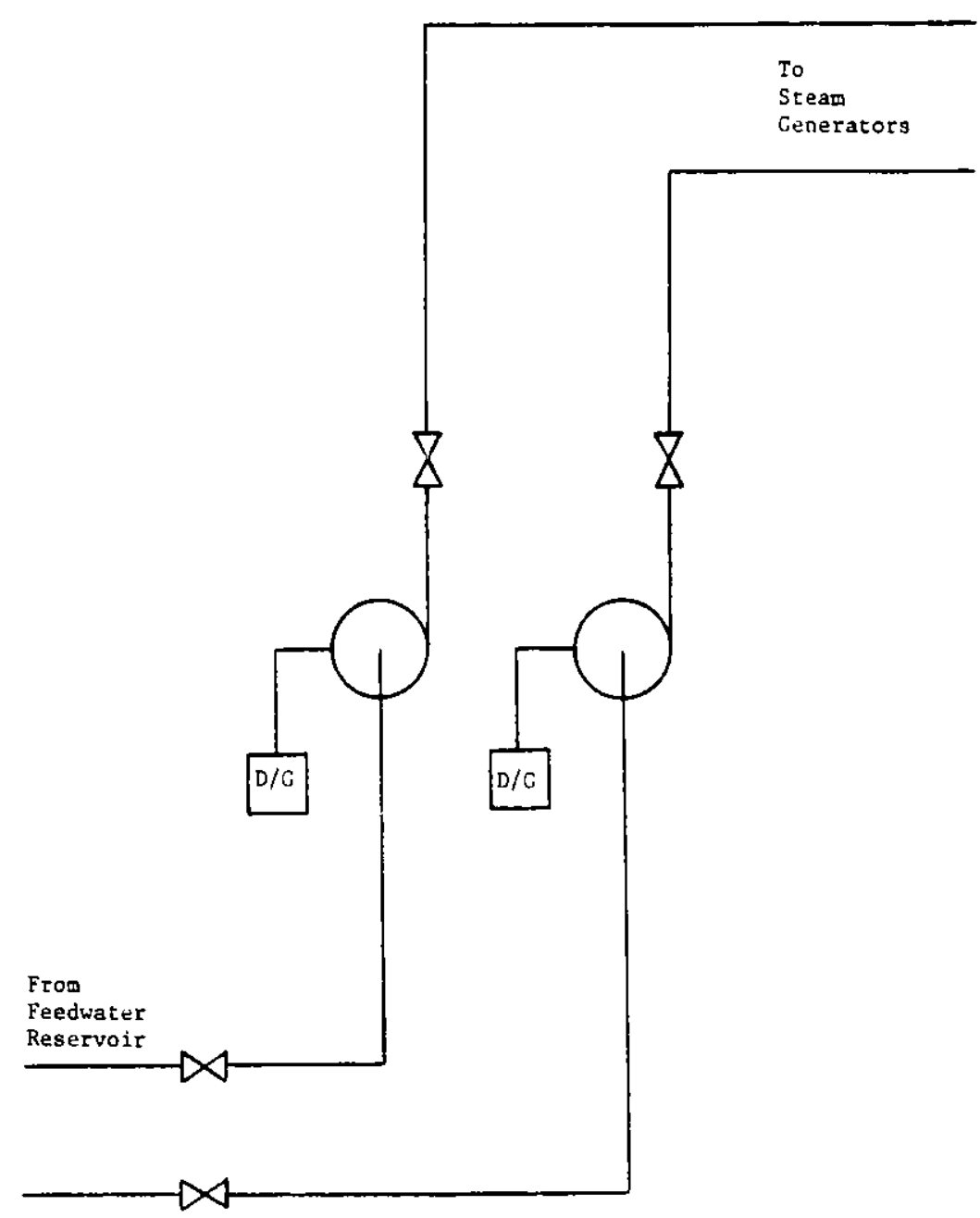

lig. 14

System Drawing for case III

a. Duration of Redundancy Test Period and Fraction of Downtime. The two valves and pump are down during testing, and since the test-period duration of the Diesel generator is the same as that of the pump, $1.4 \mathrm{~h}$ (Ref. 10 , Table III 5-1), it is as sumed that the generator is tested concurrently with the pump; $\tau_{t}=2.26 \mathrm{~h}, \mathrm{q}_{0}=1.0$.

b. Duration of Redundancy Repair Period. To calculate the repair period duration, $\tau_{r}$, we must calculate the $R$ term for each component. As for the $\gamma_{e}, \gamma_{r}, \rho_{u}$, and $\rho_{f}$ terms in Sec. V.C.l.b, each is divided by the number of components in the redundancy. This yields

$$
Y_{e}=\gamma_{r}=2.5 \times 10^{-4}, \rho_{u}=5 \times 10^{-4}, \rho_{f}=5.28 \times 10^{-3} \text {, }
$$

and

$$
\tau_{r_{\text {eff }}}=\frac{2 R_{v^{\top} r v}+R_{p}{ }^{\top} r_{p}+R_{D} / G^{\top} r D / G}{2 R_{v}+R_{p}+R_{D} / G}=20.0 \mathrm{~h} .
$$

The value of $T \mathrm{rD} / \mathrm{G}=21 \mathrm{~h}$ was obtained from Ref. 10, Table III 5-3. 
c. Redundancy Failure Rate. The redundancy failure rate is the sum of the failure rates of the components in the redundancy:

$$
\lambda_{\text {eff }}=2 \lambda_{\mathrm{V}}+\lambda_{\mathrm{p}}+\lambda_{\mathrm{D} / \mathrm{G}}=4.33 \times 10^{-5} / \mathrm{h},
$$

where $\lambda_{\mathrm{V}}$ and $\lambda_{\mathrm{p}}$ were evaluated previously and $\lambda_{\mathrm{D}} / \mathrm{G}$ is derived from the fact that $Q_{D / G}=\lambda T=3.0 \times 10^{-2}$.

d. ICARUS Results for Random Testing Scheme. The results for the random testing scheme for Case III are shown in Table XX. This is a very unreliable system due to the high failure rate of the Diesel generators. This is evident in the contribution of random failures to the average unavailability. It now dominates and drives the optimum test interval down to 13.4 Jays. Also, since the repair time for the Diesel generator is long, the repair contribution becomes significant $(6.8 \%)$.

TABLE XX. ICARUS Results for Case III

\begin{tabular}{|c|c|c|c|}
\hline $\begin{array}{l}\text { Testing } \\
\text { Scheme }\end{array}$ & $\begin{array}{l}\text { Completely } \\
\text { Random }\end{array}$ & $\begin{array}{l}\text { Uniformly } \\
\text { Staggered }\end{array}$ & $\begin{array}{c}\text { Nearly } \\
\text { Simultaneous }\end{array}$ \\
\hline $\begin{array}{l}\text { Average } \\
\text { Unavailability }\end{array}$ & $5.43 \times 10^{-4}$ & $4.83 \times: 0^{-4}$ & $7.20 \times 10^{-4}$ \\
\hline $\begin{array}{l}\text { Optimum } \\
\text { Test } \\
\text { Interval, h }\end{array}$ & 320.5 & 201.7 & 214.8 \\
\hline $\begin{array}{l}\text { Average } \\
\text { Unavailability } \\
\text { at TOPTIMUM }\end{array}$ & $3.36 \times 10^{-4}$ & $2.41 \times 10^{-4}$ & $3.85 \times 10^{-4}$ \\
\hline $\begin{array}{l}\text { Unavailability } \\
\text { due to Testing }(\%)\end{array}$ & $\begin{array}{c}1.32 \times 10^{-4} \\
(24.3)\end{array}$ & $\begin{array}{c}1.16 \times 10^{-4} \\
(24.1)\end{array}$ & $\begin{array}{c}2.20 \times 10^{-4} \\
(30.5)\end{array}$ \\
\hline $\begin{array}{l}\text { Unavailability } \\
\text { due to Repair }(\%)\end{array}$ & $\begin{array}{c}3.70 \times 10^{-5} \\
(6.8)\end{array}$ & $\begin{array}{c}3.59 \times 1 l^{-5} \\
(7.4)\end{array}$ & $\begin{array}{c}3.83 \times 10^{-5} \\
(5.3)\end{array}$ \\
\hline $\begin{array}{l}\text { Unavailability } \\
\text { due to Random } \\
\text { Failures }(\%)\end{array}$ & $\begin{array}{c}3.75 \times 10^{-4} \\
(68.9)\end{array}$ & $\begin{array}{c}3.31 \times 10^{-4} \\
(68.5)\end{array}$ & $\begin{array}{c}4.62 \times 10^{-4} \\
(64.2)\end{array}$ \\
\hline
\end{tabular}

Figure 15 is a plot of average unavailability versus test interval for the testing scheme. At short test intervals, the slope of the unavailability curve is not as steep as in the previous two cases since testing does not contribute as greatly as before. 


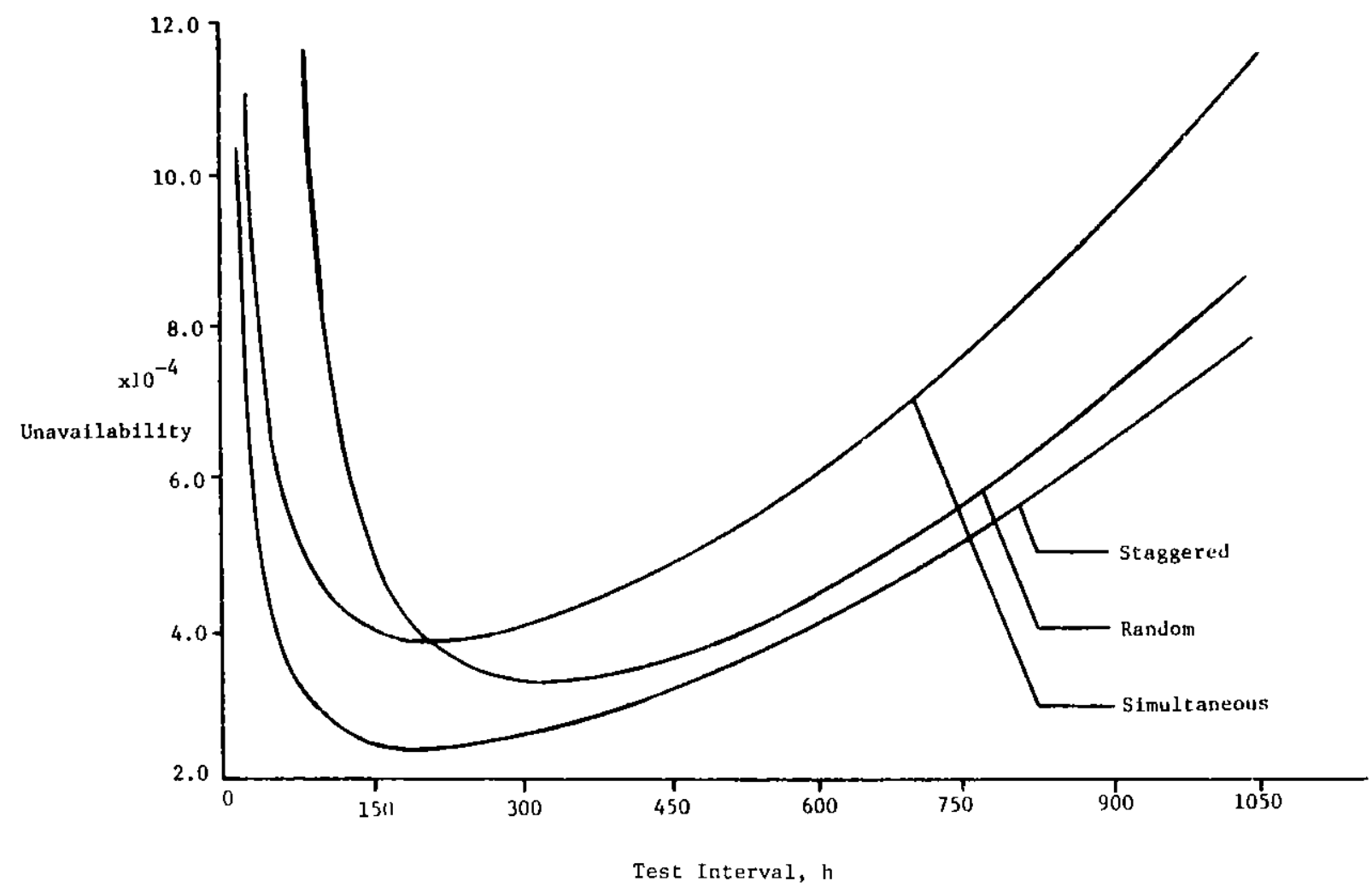

Fig. 15. Average Unavailability vs Test Interval for Cise III

e. ICARUS Results for Uniformly Staggered I esting. The results for the uniformly staggered testing scheme are also listed in Table XX. The average unavailability is also high for this scheme and system, most of it again due to random failure.

Figure 15 shows the curve for average unavailability versus test interval. The optimum test interval is only 8.4 days and would seem to be unreasonable, even though the reliability of the system could be doubled. Manpower to perform the tests in such short intervals would be scarce, given the other tests and repairs that must be performed.

f. ICARUS Results for Nearly Simultaneous Testing. Table XX shows the results for the nearly simultaneous testing scheme. This is again the least desirable testing scheme for test intervals greater than $216 \mathrm{~h}$.

Figure 15 is a plot of average unavailability versus test interval. The optimum test interval is short, 9.0 days, but reduces the average unavailability by a factor of 2.03. At short test intervals, the simultaneous scheme has a smaller average unavailability than the random scheme.

\section{Summary of Results}

The pertinent data from all three cases are summarized in Table XXI. For the auxiliary feedwater system, the $1 / 3$ configuration is 
TABLE XXI. Summary of Data from All Cases

\begin{tabular}{|c|c|c|c|c|c|c|c|c|c|}
\hline \multirow[b]{2}{*}{ Testing Scheme } & \multicolumn{3}{|c|}{ Case 1} & \multicolumn{3}{|c|}{ Case 11} & \multicolumn{3}{|c|}{ Case III } \\
\hline & Random & Staggered & Simultaneous & Random & Staggered & Simultaneous & Random & Staggered & Simultaneous \\
\hline $\begin{array}{l}\text { Average Unavail- } \\
\text { ability at } T=720 \mathrm{n}\end{array}$ & $3.2 \times 10^{-7}$ & $1.8 \times 10^{-7}$ & $3.8 \times 10^{-7}$ & $4.7 \times 10^{-5}$ & $3.7 \times 10^{-5}$ & $5.1 \times 10^{-5}$ & $5.4 \times 10^{-4}$ & $4.8 \times 10^{-4}$ & $7.2 \times 10^{-4}$ \\
\hline $\begin{array}{l}\text { Optimum Test } \\
\text { Interval, h }\end{array}$ & 1624.4 & 1183.2 & 1381.3 & 1624.4 & 1403.1 & 1512.7 & 320.5 & 201.7 & 214.8 \\
\hline $\begin{array}{l}\text { Average Unavail- } \\
\text { ability at Optimum } \\
\text { Test Interval }\end{array}$ & $2.0 \times 10^{-7}$ & $1.6 \times 10^{-7}$ & $3.2 \times 10^{-7}$ & $3.5 \times 10^{-5}$ & $3.2 \times 10^{-5}$ & $4.1 \times 10^{-5}$ & $3.4 \times 10^{-4}$ & $2.4 \times 10^{-4}$ & $3.9 \times 10^{-4}$ \\
\hline $\begin{array}{l}\text { Average Unavail- } \\
\text { ability Relative } \\
\text { to Case III }\end{array}$ & & 0.0007 & & & 0.13 & & & 1.0 & \\
\hline
\end{tabular}


206 times more available than the $1 / 2$ system (Case I versus Case II) for the 720 - $h$ test interval and staggered testing. If the Diesel generator is needed in the $1 / 2$ system, the system suffers an availability drop of a factor of 7.5 . The Diesel generators are the most unreliable components in the series.

With data from Table XXI, a yearly average unavailability can be calculated for the three testing schemes. If the probability of having both off site power and steam available to run the pumps (Case I) can be estimated to be about 0.70 and the probability of losing offsite power is taken to be $0.1 / \mathrm{yr}^{3}$, then the probability of Case II is calculated to be 0.27 , which is simply the probability of no steam $(0.30)$ times the probability of offsite power $(0.90)$. The probability of Case III is the remaining 0.03 .

For each testing scheme, the yearly average unavailability, as suming a test interval of $720 \mathrm{~h}$ is:

Random testing,

$\bar{U}_{y r}=0.70\left(3.2 \times 10^{-7}\right)+0.27\left(4.7 \times 10^{-5}\right)+0.03\left(5.4 \times 10^{-4}\right)=2.91 \times 10^{-5}$.

Uniformly staggered testing,

$\overline{\mathrm{U}}_{\mathrm{yr}}=0.70\left(1.8 \times 10^{-7}\right)+0.27\left(3.7 \times 10^{-5}\right)+0.03\left(4.8 \times 10^{-4}\right)=2.45 \times 10^{-5}$.

Nearly simultaneous testing,

$\overline{\mathrm{U}}_{\mathrm{yr}}=0.70\left(3.8 \times 10^{-7}\right)+0.27\left(5.1 \times 10^{-5}\right)+0.03\left(7.2 \times 10^{-4}\right)=3.56 \times 10^{-5}$.

With the most reliable scheme, the average unavailability is $2.45 \times 10^{-5}$, of which $59 \%$ is due to the Case III unavailability. With the plots in Figs. 12, 13, and 15, an optimum test interval for the yearly average unavailability can be found. The yearly optimum test interval is $473 \mathrm{~h}$ for the staggered testing (most reliable) scheme and gives rise to an average unavailability of $2.22 \times 10^{-5}$, a factor of 1.10 times less than the average unavailability with a $720-h$ test interval.

\section{Sensitivity of ICARUS Results}

The ICARUS code was incorporated as a set of subroutines into the probabilistic response surface code $\mathrm{PROSA}^{8}$ in order to study the sensitivity of the ICARUS results to the various input parameters. To perform this analysis, PROSA assigns selected probability distributions to each parameter being evaluated, and then selects combinations of these input parameters to determine the sensitivity of the results or consequences to the input.

Only Case III results were analyzed, since its contribution to the average unavailability was the greatest. The user-defined consequences in 
this case were the averag unavailability and the optimum test interval. The log normal distribution was assigned to all the input parameters except for $q_{g}$, which was held constant at 1.0 . For the parameters $\gamma_{r}, \gamma_{e}, \rho_{f}, \rho_{u}$, and $\lambda$, $90 \%$ confidence limits within a factor of 3 from the nominal values used in Sec. V.C. 3 were chosen. For $\tau_{r}$ and $\tau_{t}, 99.9 \%$ confidence limits and for $T$ a nearly $100 \%$ confidence limit were used within the same factor of 3 from nominal.

For Case III, uniformly staggered testing, the average unavailability was sensitive to the input parameters in the order listed in Table XXII. The numbers in the third column are the magnitudes of the sensitivity relative to the least sensitive parameter. Note that the $\rho_{u}, Y_{r}$, and $\rho_{f}$ parameters are third, fifth, and sixth, respectively, in order of sensitivity. This shows the importance of the new failure modes included in the equations derived earlier. For Case I and II, these modes are even more significant, since for these the random failures $(\lambda T)$ are not dominating as in Case III.

TABLE XXII, Results of Sensitivity Studies

\begin{tabular}{|c|c|c|c|c|c|c|}
\hline \multirow[b]{2}{*}{ Ranking } & \multicolumn{2}{|c|}{$\overline{\mathrm{U}}$ Sensitivity } & \multicolumn{2}{|c|}{${ }^{\mathrm{T}}$ OPTIMUM Sensitivity } & \multicolumn{2}{|c|}{$\overrightarrow{\mathrm{U}}_{1 / 2} / \overline{\mathrm{U}}_{1 / 3}$ Sensitivity } \\
\hline & Parameter & $\begin{array}{l}\text { Relative } \\
\text { Importance }\end{array}$ & Parameter & $\begin{array}{c}\text { Relative } \\
\text { Importance }\end{array}$ & Parameter & $\begin{array}{l}\text { Relative } \\
\text { Importance }\end{array}$ \\
\hline 1 & $\lambda$ & 793.0 & $\lambda$ & 188.5 & $\lambda$ & 1011.1 \\
\hline 2 & $\mathrm{~T}$ & 134.1 & $\tau_{t}$ & 46.6 & $\mathrm{~T}$ & 198.1 \\
\hline 3 & $\rho_{u}$ & 85.9 & $\rho_{u}$ & 37.4 & $\rho_{u}$ & 132.0 \\
\hline 4 & $\tau_{t}$ & 47.0 & $\gamma_{r}$ & 17.0 & $\tau_{t}$ & 67.6 \\
\hline 5 & $\gamma_{r}$ & 42.1 & $\rho_{f}$ & 6.3 & $\gamma_{\mathrm{r}}$ & 48.8 \\
\hline 6 & $\rho_{f}$ & 35.5 & $\tau_{r}$ & 4.2 & $\rho_{f}$ & 45.8 \\
\hline 7 & $\tau \mathbf{r}$ & 17.7 & $\gamma_{e}$ & 1.0 & $\tau_{\mathbf{r}}$ & 17.5 \\
\hline 8 & $\gamma_{e}$ & 1.0 & $\mathrm{~T}$ & 0 & $\gamma_{\mathrm{e}}$ & 1.0 \\
\hline
\end{tabular}

The optimum test interval of $201 \mathrm{~h}$ had a standard deviation of about the same magnitude. The order of the sensitivity of the optimum test interval to the input is also listed in Table XXII. As shown, the $\rho_{u}, \gamma_{r}$, and $\rho_{f}$ terms were the third, fourth, and fifth most significant terms in determining the optimum test interval.

A final analysis was performed in which the ratio of the average unavailability of the Case III system $(1 / 2)$ to the same type of system with three redundancies ( $1 / 3$ system with Diesel generators) was found to be 56 with a standard deviation of 46 . In other words, increasing the system from $1 / 2$ to $1 / 3$ would increase the reliability by a factor of 56 , but the uncertainty of this value is high (standard deviation $=46$ ). 
The order of sensitivity of the ratio to the input is listed in columns 6 and 7 of Table XXII. Note that this is the same order as for the average unavailability of the $1 / 2$ system.

D. Summary

The results of the analysis show that the average unavailability of the auxiliary feedwater system over a one-year period is $2.45 \times 10^{-5}$ for the most reliable testing scheme (uniformly staggered testing). Most of this unavailability is due to the Diesel generators being part of the system when no outside power source is available.

The sensitivity analysis of the input shows that the new failure modes introduced in this report are important parameters when evaluating the average unavailability of the auxiliary feedwater system. 


\section{SUMMARY AND CONCLUSIONS}

A. Summary

Standby safety systems at nuclear power plants are called upon to function if an emergency situation develops. The system, however, may fail to respond due to mechanical breakdown or human failure. It is therefore of interest to be able to calculate the average unavailability of the system in order to quantify this probability of failure to respond.

Efforts have been made in the past to develop methods to calculate the average unavailability of a system as a function of various failure modes and testing practices. For the most part, however, these efforts were either severely limited in the number and type of failures that could be treated, or did not allow for the calculation of other parameters of interest related to the average unavailability, such as the optimum test interval.

In order to treat a variety of failure modes and to apply the treatment to the calculation of the average unavailability in such a way that the optimum test interval of the system could also be easily calculated, analytical equations for all $m$-out-of $-n(m / n, 1 \leq m \leq n \leq 4)$ safety systems were developed for completely random, uniformly staggered, and nearly simultaneous testing schemes. A computer code, ICARUS, was written to incorporate these equations in such a way that the contributions of testing, repair, and random failures could also be calculated. Since the average unavailability equations were analytically derived and then programmed, the running time of the computer code is short ( $<1$ s for analysis of all $m / n$ systems with $1 \leq m \leq n \leq 4)$.

To verify the coding in ICARUS, comparison runs were made between ICARUS and FRANTIC using options common to both codes. For single component redundancy systems, the results showed adequate agreement for both uniformly staggered and nearly simultaneous testing.

Since most systems have redundancies made up of more than one component, it was clear that some special treatment would be needed in order to apply ICARUS to these systems. In this work, the multicomponent redundancies were represented by a single effective component. Each input parameter was modified to reflect the "effective" component characteristics.

Comparison runs were then made with FRANTIC for systems having two valves in series making up each redundancy. For the uniformly staggered testing scheme, the agreement was again acceptable for all $\mathrm{m} / \mathrm{n}(1 \leq \mathrm{m} \leq \mathrm{n} \leq 4)$ systems. In the comparison of the nearly simultaneous testing scheme, the highly redundant system comparisons displayed large errors. After checking against a hand calculation of a $1 / 3$ system, we concluded that FRANTIC cannot suitably treat these highly redundant systems, which are nearly simultaneously tested. 
Finally, ICARUS was used to calculate the average unavailability of an auxiliary feedwater system. Data for testing practice and failure modes was obtained from the literature in order to make a realistic assessment of the unavailability. The auxiliary feedwater system can take on various configurations, depending upon the presence of steam and offsite power. A yearly average unavailability for the system was calculated taking into account different configurations. The uniformly staggered testing scheme was found to be the most desirable. Also, the configuration that has Diesel generators driving the electrical pumps accounts for $59 \%$ of the yearly average unavailability.

A sensitivity analysis using the PROSA code was performed to determine which of the ICARUS input parameters were the most important. We found that the failure modes in ICARUS that are not in J'RANTIC are important enough so that their omission in any unavailability analysis may lead to inaccurate results.

B. Recommendations for Future Work

The equations developed for the ICARUS code represent an extensive development of average unavailability equations for redundant systems. However, additions could be made to the code that would even more realistically model the system in question. The most important of these is the treatment of common-mode or common-cause failure, since common-mode failure has played an important part in system failures in the past.

A second task that might be undertaken would be the modification of the ICARUS code to handle multicomponent redundancies directly. Each redundancy could be treated as a set of miniature systems whose average unavailabilities could be calculated using the existing ICARUS equations. Depending upon the configuration of the components in the redundancy, the average unavailability of the redundancy and then of the system could be calculated. To be able to calculate the optimum test interval directly, the calculation of the average unavailability should be in terms of coefficients of the powers of the test interval $\mathrm{T}$ (see Chapter III). The calculation of the optimum test interval is one of the main features found in ICARUS.

Another line of development includes modifications in the FRANTIC code such that the main features of the ICARUS code, as well as the models of Ref. 7, can be merged to a general availability-analysis code. 
APPENDIX A

Linearization of $\exp (-\lambda s)$

In order to simplify the average unavailability expressions, an approximation for $\exp (-\lambda s)$ would be in order. Expanding exp $(-\lambda s)$ in a Taylor series about the origin (Maclaurin series) yields

$$
\exp (-\mathbf{s})=1-\lambda s+\lambda^{2} s^{2} / 2 !-\lambda^{3} s^{3} / 3 !+\lambda^{4} s^{4} / 4 !-\ldots
$$

Taking the first two terms of the expansion, we can write $\exp (-\lambda s)$ as

$$
\exp (-\lambda s)=1-\lambda s
$$

The unavailability at any time $s$ can be found from

or

$$
\left.\begin{array}{l}
U(s)=1-\exp (-\lambda s) \simeq 1-(1-\lambda s) \\
U(s)=\lambda s
\end{array}\right\} .
$$

Table A.l shows the errors incurred using this approximation. For values of $\lambda \mathrm{s}$ less than $10^{-3}$, which is typical for this study, the error is less than $0.1 \%$.

TABLE A.l. $\lambda s$ vs $l-\exp (-\lambda s)$

\begin{tabular}{ccc}
\hline$\lambda \mathrm{s}$ & $1-\exp (-\lambda \mathrm{s})$ & Percent Error ${ }^{\mathrm{a}}$ \\
\hline 1.0 & 0.6321 & 58.20 \\
0.5 & 0.3935 & 27.07 \\
$1.0 \times 10^{-1}$ & $9.52 \times 10^{-2}$ & 5.08 \\
$5.0 \times 10^{-2}$ & $4.88 \times 10^{-2}$ & 2.52 \\
$1.0 \times 10^{-2}$ & $9.95 \times 10^{-3}$ & 0.501 \\
$5.0 \times 10^{-3}$ & $5.98 \times 10^{-3}$ & 0.250 \\
$1.0 \times 10^{-3}$ & $9.995 \times 10^{-4}$ & 0.050 \\
$5.0 \times 10^{-4}$ & $4.999 \times 10^{-4}$ & 0.025 \\
$1.0 \times 10^{-4}$ & $9.9995 \times 10^{-5}$ & 0.005 \\
\hline $\mathrm{a}_{\lambda \mathrm{s}-[1-\exp (-\lambda \mathrm{s})]} \times 100 \%$. &
\end{tabular}




\section{APPENDIX B}

\section{ICARUS Users' Manual}

\section{Program Summary}

The program calculates the unavailability characteristics of a periodically tested standby safety system with $m$-out-of- $n$ redundancy $(1 \leq m \leq n \leq 4)$.

The main functions of the program are to calculate the average unavailability, the optimum test interval, and fractional unavailabilities due to testing, repair, and random failures for three different testing strategies. It will also calculate the average unavailability as a function of the test interval at each point in the range $0.2 \mathrm{~T}_{\mathrm{OPTIMUM}}$ to $1.8 \mathrm{~T}$ OPTIMUM at intervals of $0.2 \mathrm{~T}_{\text {OPTIMUM }}$.

The code can be used to evaluate sensitivities to different failure modes and data as well as current testing/repair strategies to determine if changes to these strategies will optimize the system availability.

2. Program Abstract

a. Name of Program. ICARUS

b. Computer for Which Program Is Designed. IBM 370/195

c. Description of Problem. The problem is to determine the average unavailability of a standby system with $\mathrm{m} / \mathrm{n}$ redundancy, given that it can fail due to testing, maintenance, mechanical failure, or human error. The $\mathrm{m} / \mathrm{n}$ redundancy means that the system has $n$ parallel components, each with $100 \% / \mathrm{m}$ capacity; i.e., operation of $\mathrm{m}$ components is needed to perform the. safety function.

ICARUS first calculates the average unavailability, optimum test interval, and average unavailability at the optimum test interval, given the system configuration and testing schemn. If desired, the code then calculates the average unavailabilities at points around the optimum test interval and also calculates the unavailability contributions due to testing, repair, and random failures.

d. Method of Solution. As input data, the program needs the system configuration ( $m$ and $n$ ), testing scheme, testing and repair paramcters, and values for the testing, repair, and standby failure modes. Using these data, the program calculates the average unavailability for the system and solves for the optimum test interval of the system using the unavailability equation. Since the analytical cquations for each system and testing strategy are programmed into the code, computational time is very short. 
If desired by the user, the code calculates the average unavailability in the interval $0.2 \mathrm{~T}_{\text {OPT IMUM }}$ to $1.8 \mathrm{~T}_{\text {OPTIMUM }}$ at each $0.2 \mathrm{~T}_{\text {OPT IMUM }}$ increment. The program then calculates the contributions (numerically and in percest) that testing, repair, and random failures make to the average unavailability.

e. Restrictions on the Complexity of the Problem. The system configuration can only be $\mathrm{m} / \mathrm{n}$ with $1 \leq \mathrm{m} \leq \mathrm{n} \leq 4$, which is sufficient for most safety systems currently in use. The three testing strategies allowed in the code are:

Random

Uniformly staggered

Nearly simultaneous.

In the random testing scheme, the componants are tested at randomly shifted times throughout the test interval. The uniformly staggered testing scheme has the component tests being performed at equally spaced times throughout the test interval. Finally, in the nearly simultaneous testing schene, all the components a re tested consecutively at the beginning of the test interval. .

The equations assume only one component per redundancy. However, redundancies with several components in series can be analyzed by properly defining the failure data for a series system in terms of the data for individual components.

f. Typical Running Time. Typical running time for all $\mathrm{m} / \mathrm{n}(1 \leq \mathrm{m}$ $\leq \mathrm{n} \leq 4)$ systems and all three testing schemes is $<1$ s on an IBM 370/195.

g. Unusual Features of the Program. The analytical unavailability equations for all 28 systems (1/1 system plus nine systems times three testing schemes per system) are separately programmed in the code. For each system, the equations (divided into coefficient equations) are polynomials with both positive and negative powers of the test interval. This allows for the calculation of the optimum test interval by taking the derivative of the average unavailability equation with respect to the test interval, setting that equal to zero, and solving for the optimum test interval. When a numerical solution is required, the Newton-Raphson method, which converges quickly for polynomial equations of this type, is used. Direct analytical equations are available for the random testing scheme.

h. Related and Auxiliary Programs. The ICARUS code can be used with the PROSA response surface analysis code to determine the sensitivities and probability distributions of the unavailabilities and optimum test intervals.

i. Status. The program is operational at ANL. 
j. References. Publications in preparation.

k. Machine Requirements. IBM 370/195; card reader; line printer.

1. Programming Language Used. FORTRAN IV.

m. Operating System. IBM System/370 Mode1 195; FORTRAN IV (G) or $(\mathrm{H})$ compiler with optimizer.

n. Other Programming or Operating Information or Restrictions. All floating-point variables are double precision.

o. Name and Establishment of Author. Dominic Sciaudone; University of Lowell, Nuclear Enginecring Department, 1 University Avenue, Lowell, Massachusetts 01854. J. K. Vaurio, Argonne National Laboratory, Bldg. 208, 9700 S. Cass Avenue, Argonne, Illinois 60439.

p. Material Available. Source decks, sample problem, control cards.

q. Category. P. General Mathematical and Computing System Routines.

r. Keywords. Availability, human errors, periodic testing, redundancy, redundant, reliability, safety system, testing, test interval, unavailability.

3. Program Use

ICARUS can be directed to perform four different tasks. The task to be executed is specified by the input variable ITASK. The tasks are described in Sec. a below. The input for each task is specified in Scc. b below. The flow charts for the program are presented in Appendix $C$, and the function of each subroutine is briefly described in Sec. c below.

\section{a. Task Descriptions}

Each exccution of the program performs any one of the following four tasks specified by the variable ITASK. After each case, the program control returns to the beginning and a new case starts (number of cases specified by NCASE).

(1) Task 1 (ITASK = 1): Selected System Unavailabilities. Calculates and prints the average unavailability, optimum test interval, and average unavailability at the optimum test interval for any system, testing scheme, and failure-mode probabilities specified by the user.

(2) Task 2 (ITASK $=2):$ Selected System Total and Fractional $\bar{U}$

(a) Performs same task as Task 1. 
(b) Calculates and prints the average unavailability of each point in the interval $0.2 \mathrm{~T}_{\text {OPTIMUM }}$ to $1.8 \mathrm{~T}_{\mathrm{OPTIMUM}}$ at an increment of $0.2 T_{\text {OPTIMUM }}$

(c) Calculates the average unavailability contributions due to testing, repair, and random failures in the form of numerical values and percents.

(3) Task 3 (ITASK = 3): All System Unavailabilities. Performs the same task as Task 1 for all $\mathrm{m} / \mathrm{n}$ systems ( $\leq \mathrm{m} \leq \mathrm{n} \leq 4$ ) and testing schemes using one set of component input values. There are 28 systems in all.

(4) Task 4 (ITASK = 4): All System Total and Fractional Unavailabilities. Performs the same task as Task 2 but for all systems and testing schemes using one set of component input values.

b. Input Instructions

Card 1

$\frac{\text { Variable }}{\text { NCASE }} \frac{\text { Columns }}{1-6} \quad \frac{\text { Format }}{16} \quad \frac{\text { Description }}{\text { Number of cases to be run }}$

Card 2

Variable

ITASK
Format

I6

\section{Description}

Task indicator (see Sec. a above)

Card 3

Variable

ITITLE

$\frac{\text { Columns }}{1-80} \quad \frac{\text { Format }}{20 \mathrm{~A} 4}$

\section{Description}

Title of case

Card 4 (Optional; only needed for ITASK = 1 or 2)

$\frac{\text { Variable }}{\text { NSYSTM }} \quad \frac{\text { Columns }}{1-6} \quad \frac{\text { Format }}{16} \quad \frac{\text { Description }}{\text { Number of systems to be run for case }}$

Card 5

Variable $\quad \frac{\text { Columns }}{1-12} \quad \frac{\text { Format }}{112}$

\section{Descriptions}

Marker for system and testing scheme. Three-digit integer. First two digits specify system $(12,1 / 2$ system, etc.) Last digit specifies testing scheme. 1 = random

2 = uniformly staggered

3 = nearly simultaneous.

Use IMARK = 100 when ITASK $=3$ or 4. 
Card 5 (Contd.)

\begin{tabular}{|c|c|c|c|}
\hline Variable & Columis & Format & Descriptions \\
\hline GAMMAE & $13-24$ & $\mathrm{D} 12.5$ & $\begin{array}{l}\text { Probability that a test causes a failure } \\
\text { that gets repaired. }\end{array}$ \\
\hline GAMMAR & $25-36$ & D12.5 & $\begin{array}{l}\text { Probability that a true diman l causes } \\
\text { a failure. }\end{array}$ \\
\hline LAMBDA & $37-48$ & D12.5 & $\begin{array}{l}\text { Failure rate per hour of component } \\
\text { redundancy. }\end{array}$ \\
\hline QZERO & $49-60$ & Dl2.5 & $\begin{array}{l}\text { Fraction of downtime for a component } \\
\text { during a test. }\end{array}$ \\
\hline RHOF & $60-72$ & Dl2.5 & $\begin{array}{l}\text { Probability that a component that had } \\
\text { failed before the test is not detected or } \\
\text { not repaired due to human testing error, } \\
\text { detection error, or human repair error. }\end{array}$ \\
\hline
\end{tabular}

Card 6

\begin{tabular}{|c|c|c|c|}
\hline Variable & Columns & Format & Descriptions \\
\hline RHOU & $1-12$ & $\mathrm{D} 12.5$ & $\begin{array}{l}\text { Probability that a test cau } \\
\text { that does not get repaired } \\
\text { next test (negligence or hi }\end{array}$ \\
\hline TAUR & $13-24$ & $\mathrm{D} 12.5$ & Duration of repair period. \\
\hline TAUT & $25-36$ & $\mathrm{D} 12.5$ & Duration of test period. \\
\hline TUP & $37-48$ & $\mathrm{Dl} 2.5$ & Duration of test interval. \\
\hline
\end{tabular}

c. Subroutine Functions

The function of each subroutine is described below.

CALC Calculates average unavailabilities and writes all results.

COMB Calculates the binomial coefficient.

FRACUN Calculates fractional unavailabilities and writes results

ITERAT Calculates optimum test interval using Newton-Raphson method.

MAIN Reads data, calculates unavailability coefficients, and performs overall routine control. 
Flow Diagrams for ICARUS

Figures C.1-C.3 present the logic used in the calculational subroutines of the ICARUS computer code.

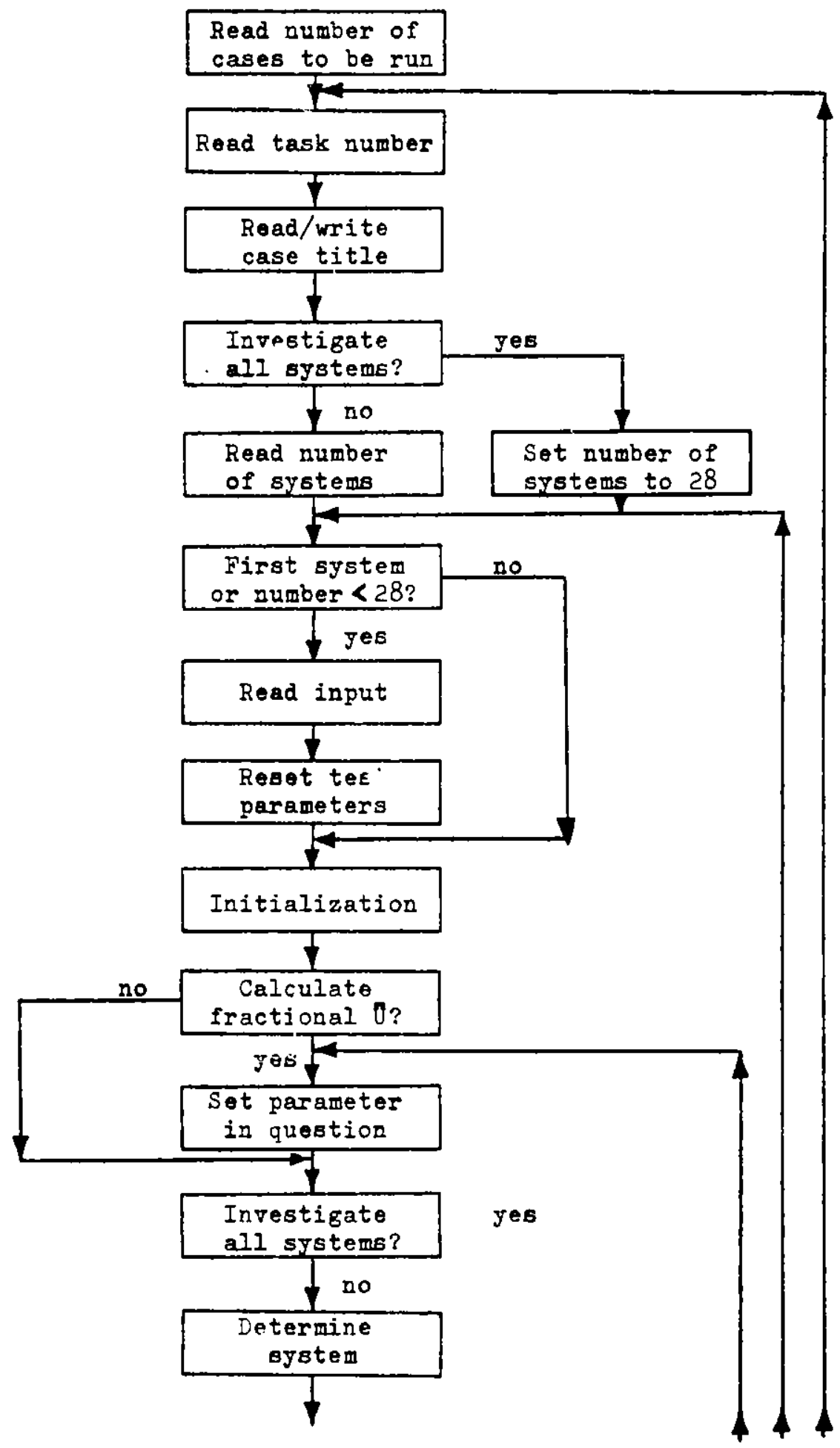

Fig. C.1. Flow Diagram for MAIN Routine 


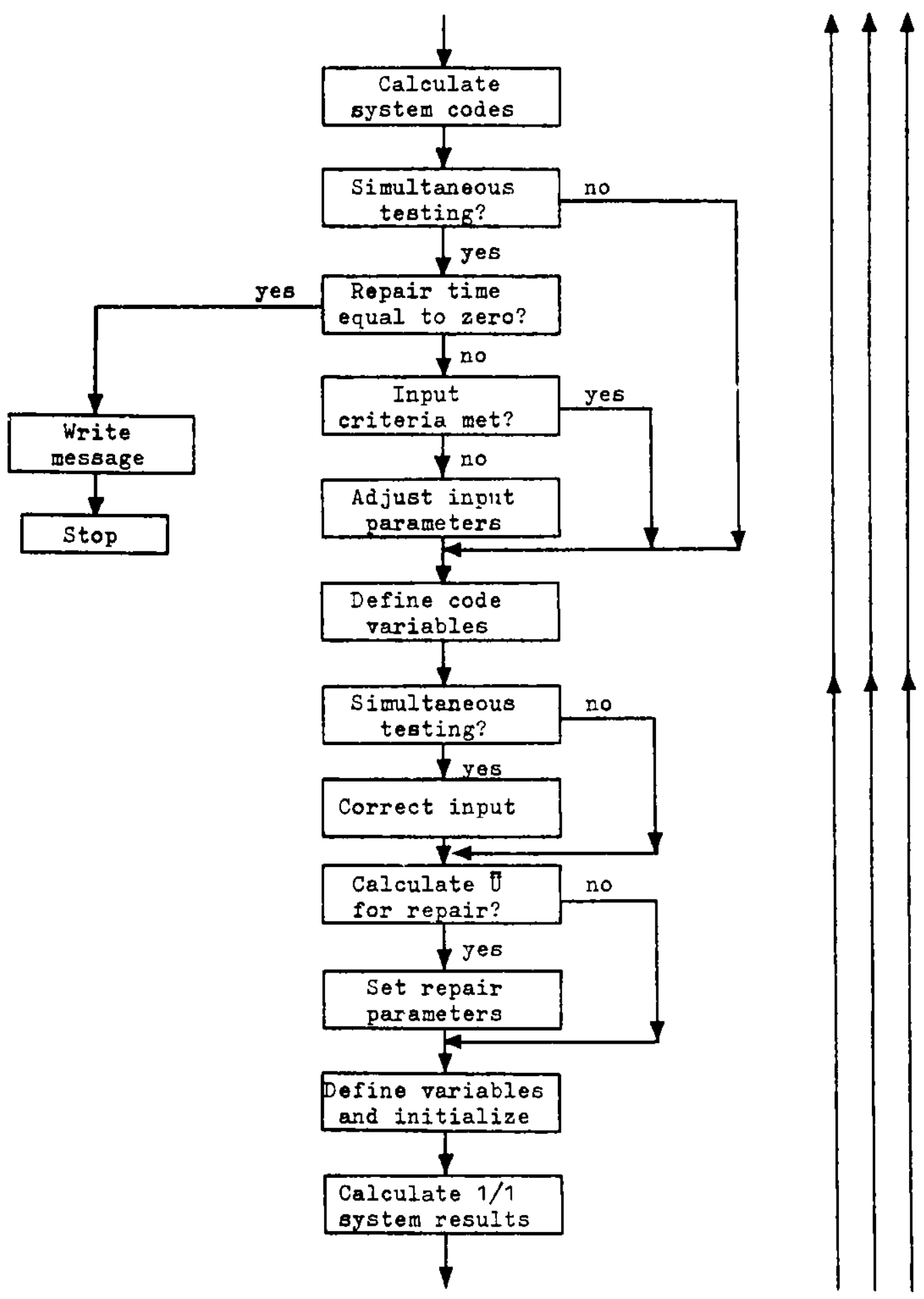

Fig. C.1 (Contd.) 


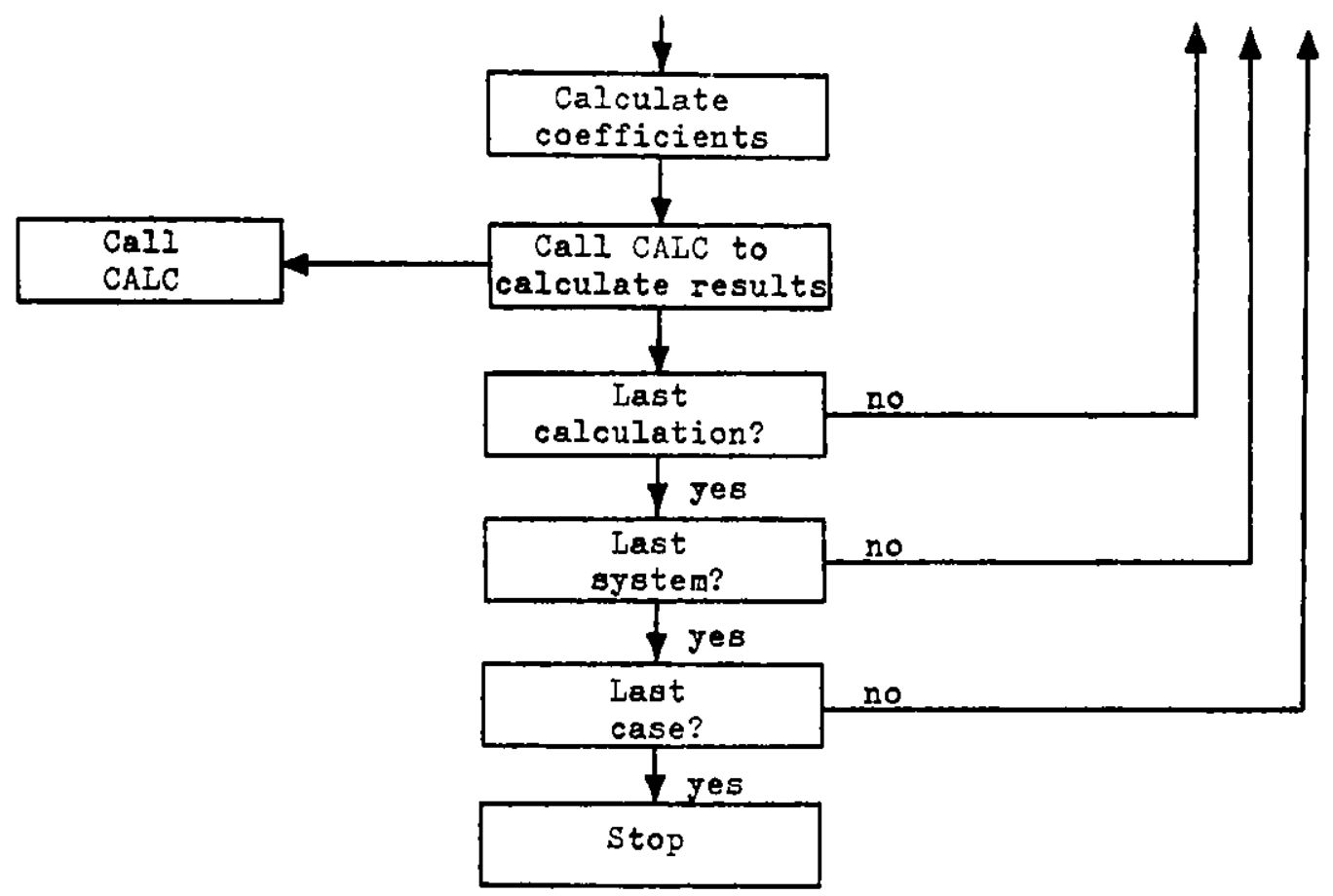

Fig. C.1 (Contd.)

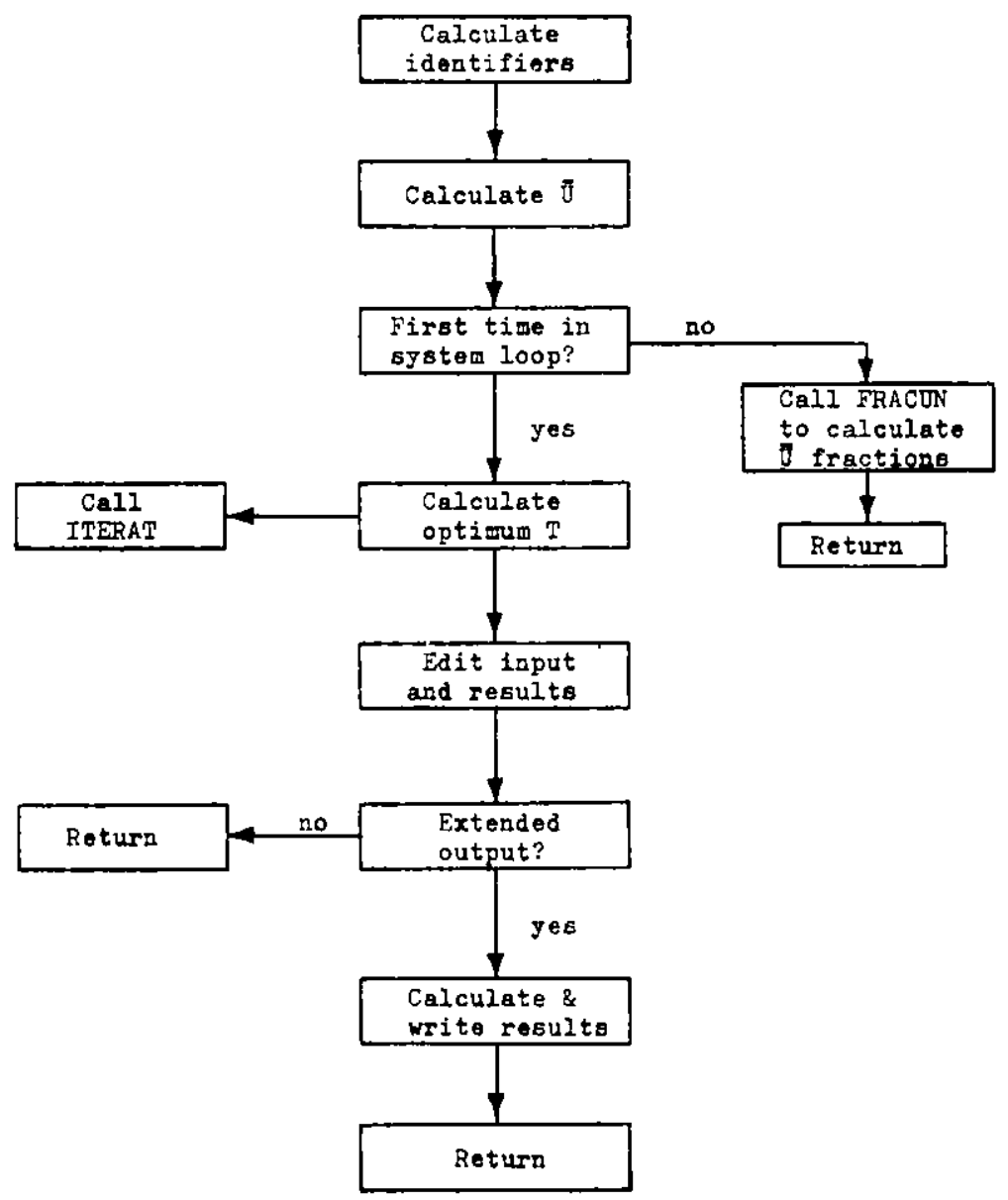

Fig. C.2. Flow Diagram for CALC Subroutine 


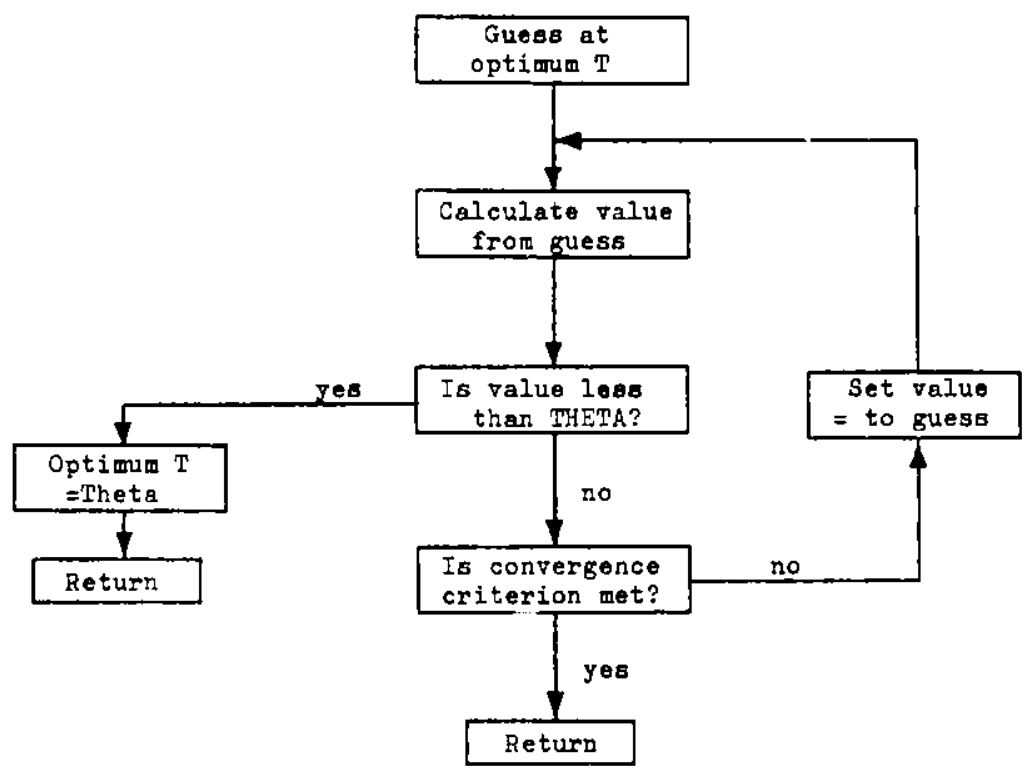

Fig. C.3. Flow Diagram for IT LRAT Subroutine 
APPENDIX D

ICARUS Program Listing

The following is a computer listing of the ICARUS computer code.

$c$
$C$
$C$

HAIN FROGRAM

IMIPLICIT REAL*8(A-H,O-Z)

REAL $\because 8$ LAMBDA

COHION/INTVAR/N, IFRK, IMRK 1, ITASK, IHR ITE, NNY 28 ), IFLAG, ISIGN, NNSYS

1, ITT

COH!ON/FLTVAR/GAH:"iAE, GAMHAR, LAMEDA, QZERO, RHOF , RHOU, TAUR, TAUT, TUP,

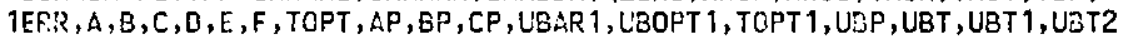

2,U3T3, UBT 12, LBT22, UBT32, THETA

DIHEAISIGN ISISTM(450), ITITLE(20)

DATA ISYSTH $109 * 0,1,10 * 0,2,3,4,7 * 0,8,9,10,7 * 0,17,18,19,77 * 0,5,6,7$,

$17 * 0,11,12,13,7 * 0,20,21,22,87 * 0,14,15,16,7 * 0,23,24,25,97 * 0,26,27,28$

$2,7 * 0$,

DATA ITITLE/20*4H

TREAD $=5$

ICASE $=0$

$E R R=1.00-03$

$c$

C INPUT DATA DEFIHITIOHS

C RICASE NULHBER OF CASES TO BE RUN - 1 CARD ONLY

C ITITLE TITLE GF CACE TO GE RUN - 1 FOR EACH CASE

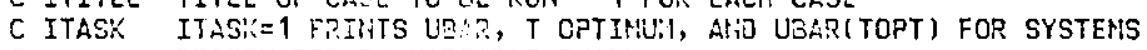

C DESIEUATCO BI VAREASLE IVIARK

C IIASK=? FIIKS ITASK=1 INFCAMATICH PLUS UBAR(TOPT) FOR VALUES

C OF . 2*TOF TO 1.8 TO:T, AL THE FPACTIOMAL UNAVAILABILITIES

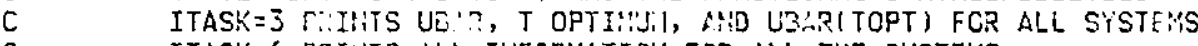

C ITASK=4 FRIITS ALL IIVONIIATICH FCR ALL THE SYSTEIIS

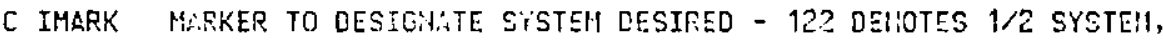

C STRCGERED TFSTING. FINST 2 IPITEGERS ARE SISTEM COHFIGUAATICN

C LAST INIEGEP IS TLSTIV SCHEHE: 1-RAHDC'! 2-STAG. 3-SIIUULT.

C IF ITAS: IS CREATER THAN 2, SET IMARK TO 100

C NOYSTM IUU: ¿ER OF SISTET:3 TO BE IHVESTIGATED (CILY IF ITASK < 3 )

C GAiviAE PRCOABILITY THAT A TEST CRUDES A FAILURE THAT GETS REPAIRED

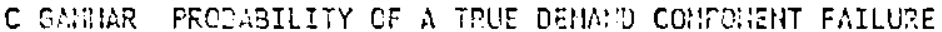

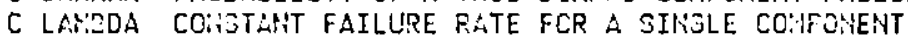

C QZERO FRACTIOH OF CC:Bi TTHE OURIHS A TEST

C RHOF FROEABILITY THAT A CO BOHEHT WHICH HAD FAILED BEFORE THE TEST

C GET REPAIRED (HLIAI TESTIHG EFFCR, DETECTIOH ERROR, OR HU:AYi

C REPAIR ERTORI

C RHOU FICOAILITY THAT A TEST CAUSES A FAILURE THAT DOES NOT GET

C

C TAUR

FEPAIRED EEFCRE THE NEXT TEST (HEGLIGET:EE OR HIUDEN TYFE FAILU?BE)

C TAUT TESTIIIG TIIEE

C TUP TESTIHG IIITERVAL

C

C READ NJMEER OF CASES TO BE RUit

C

READ(IFEAD, 1000) HCASE

c

C START OF HICASE LCOP

C

1 continue

C

C IHITIALIZE FOR CASE

$I C A S E=I C A S E+1$ 


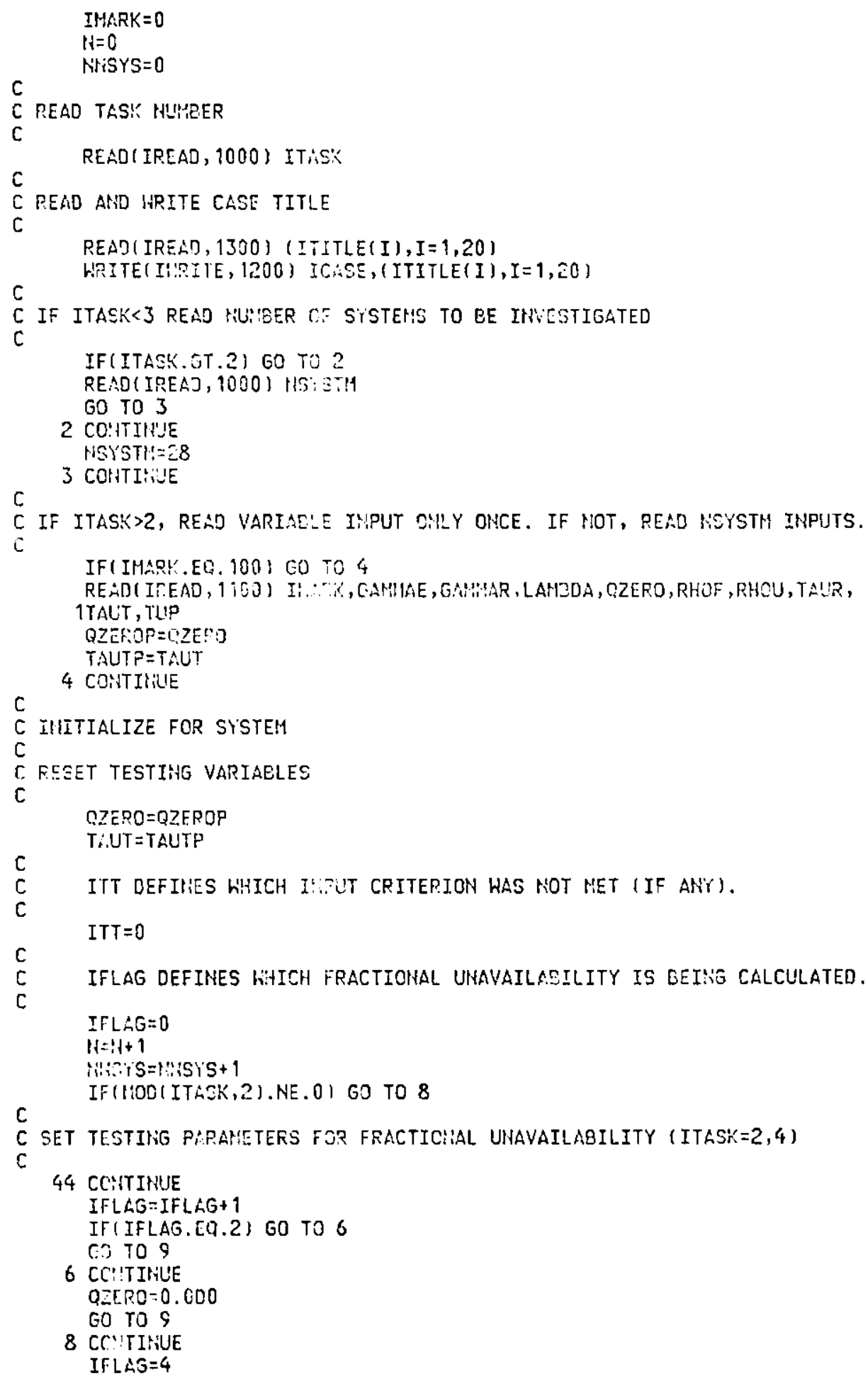


9 CONTIRUE

IF(ITASK.GT.2) GO TO 5

11: ISISTII IHAFK)

5 COHIT IHUE

$C$
$C$
$C$
$C$
$C$
$C$
$C$
$C$

NIN DEFINES SYSTEN AND TESTING SCHEME.

ISIG:N DEFINES THE TESTING SCHEME.

IV DEFIHES THE NU:B:R OF SYSTEH REDURIDANCES.

ISICH $=149(H)-(1+i(N) / 10) * 10+1$

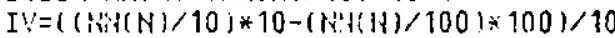

E MAKE SURE INFUT CRITERIA IRE MET FC? EACH SYSTEH. ADJUST YLLUES TO MICET

C CRITERIA IF TC:JIRLE. IF I:DT, FRIITT ERROR MESSASEE.

IF(ISIGN.HE.4) 60 TO 19

IF(TALR.LT.1.0D-50) GO TO 112

THETA $=T A U T+T, B$ R

GO TO $19,15, k, 17), \mathrm{IV}$

15 CONIII'JE

TI!U=T:ETA-2. C. TAUT

IF(THY.GE.0.600) 60 TO 19

$T R I I=0.000$

TAUT = TALR

$I T T=1$

60 TO 18

16 CONTIR:'I

Ti:U $=$ THETA-3. ก४TLUT

If(TKU.GE.0.CDO) CO to ts

$T \cdot i, j=2.608$

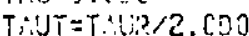

ITT $=2$

I.0 TO 18

17 COLTIIUE

TSIG THETA-4.3*TAUT

IF(TSIG.GE.0.CDO) C.O TO 19

TSIG $=0.003$

TAUT $=$ TAUR $/ 3.000$

ITT $=3$

18 CCIIIIIUE

Gi. U :RZEFCF*TSUTP/TAUT

19 CC. ITISiNE

IF(QLERO.GT.1.000) GO TO 112

$c$

C VARIABLE DEFINITICYS

THETA $=$ TAUT T TALR

GLTLTA=EHOF - HOU

CDEL $=1.0-C$ CELTA

$C L F^{\prime}=G A H M A R+R H C U / C O E L$

C

WITH SIMULTA!:COUS TESTINIG, R!IP USED IH IHTEERATIHG FROH O TO T.

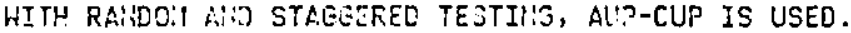

AUP $=$ OZERO $+(1,0$-QZERO $) *($ GAPHIA.R +GAP:IAE +FHOU $*(2,0-$ GDELTA $) / G D E L)-C U P$ $\therefore O H=(1.0-0 Z E R O) \div$ LANGDA 


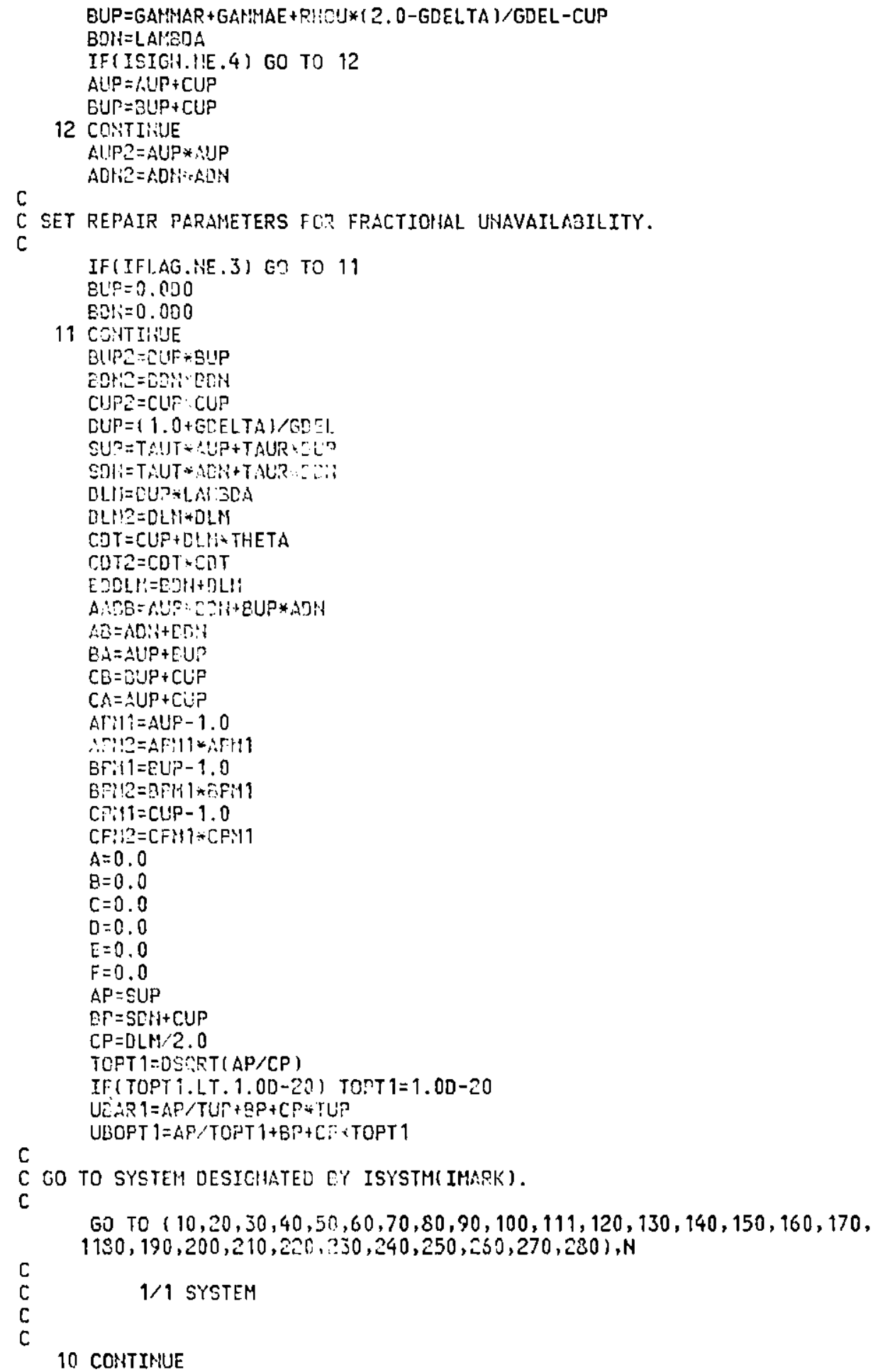




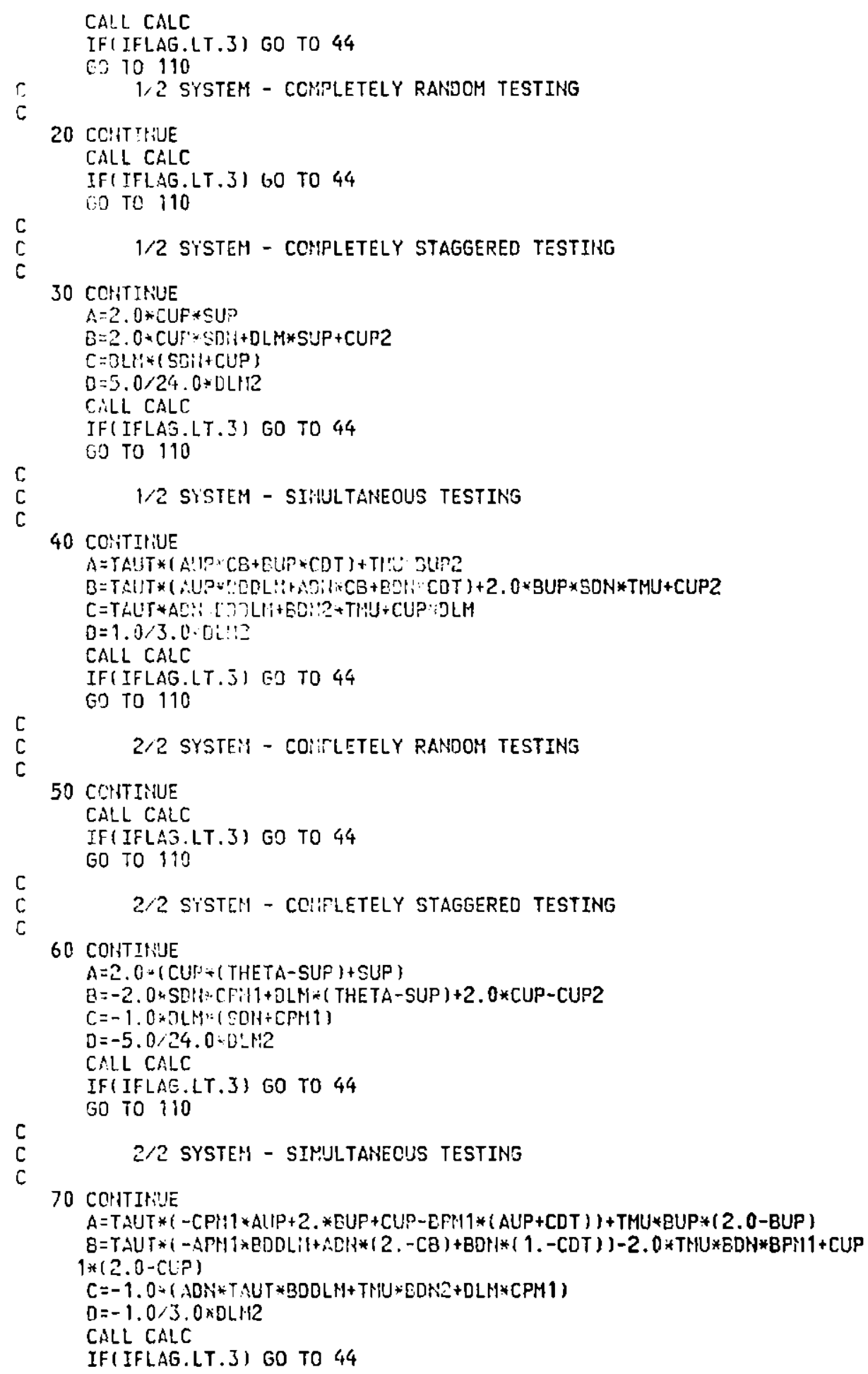




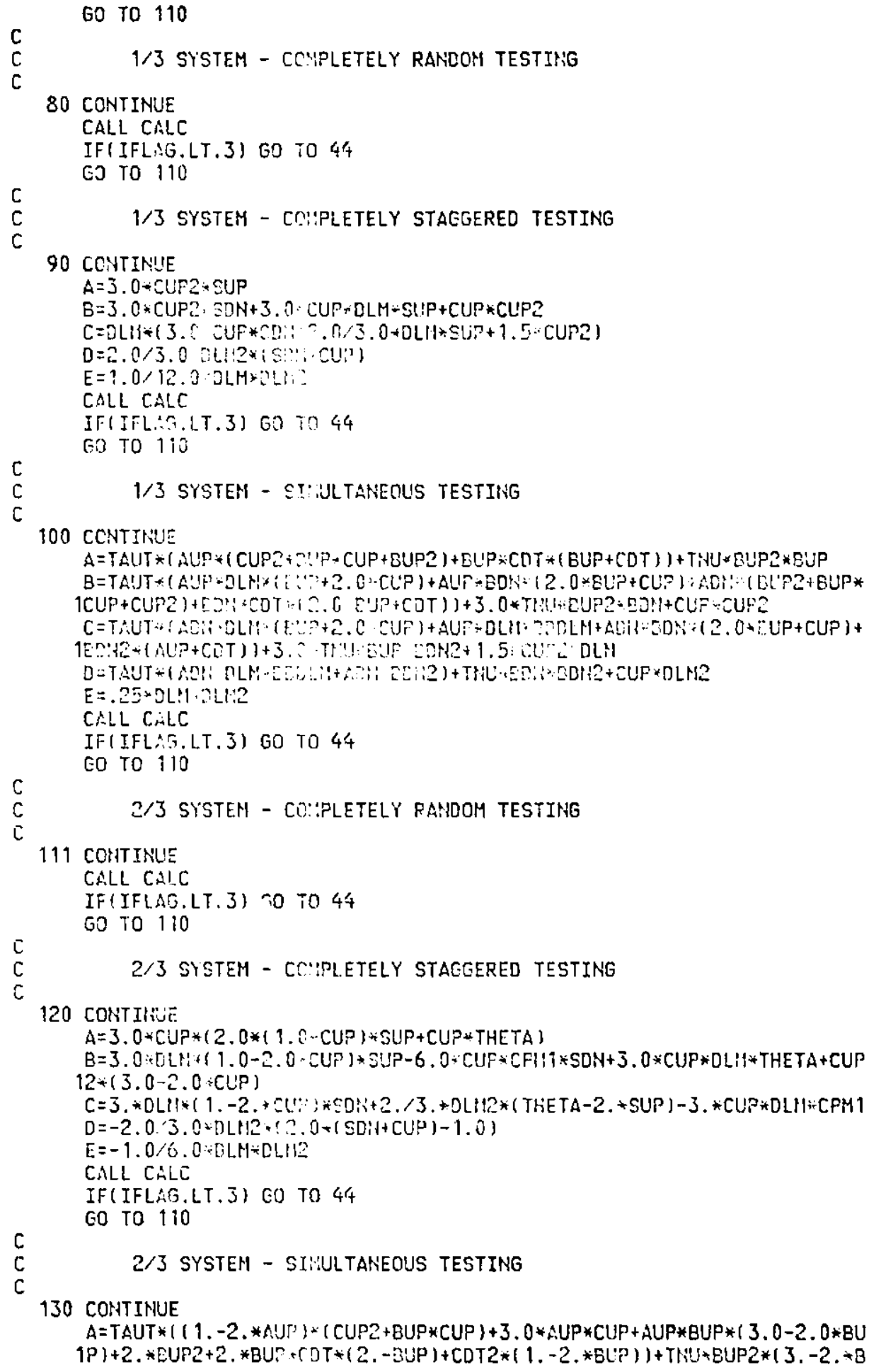




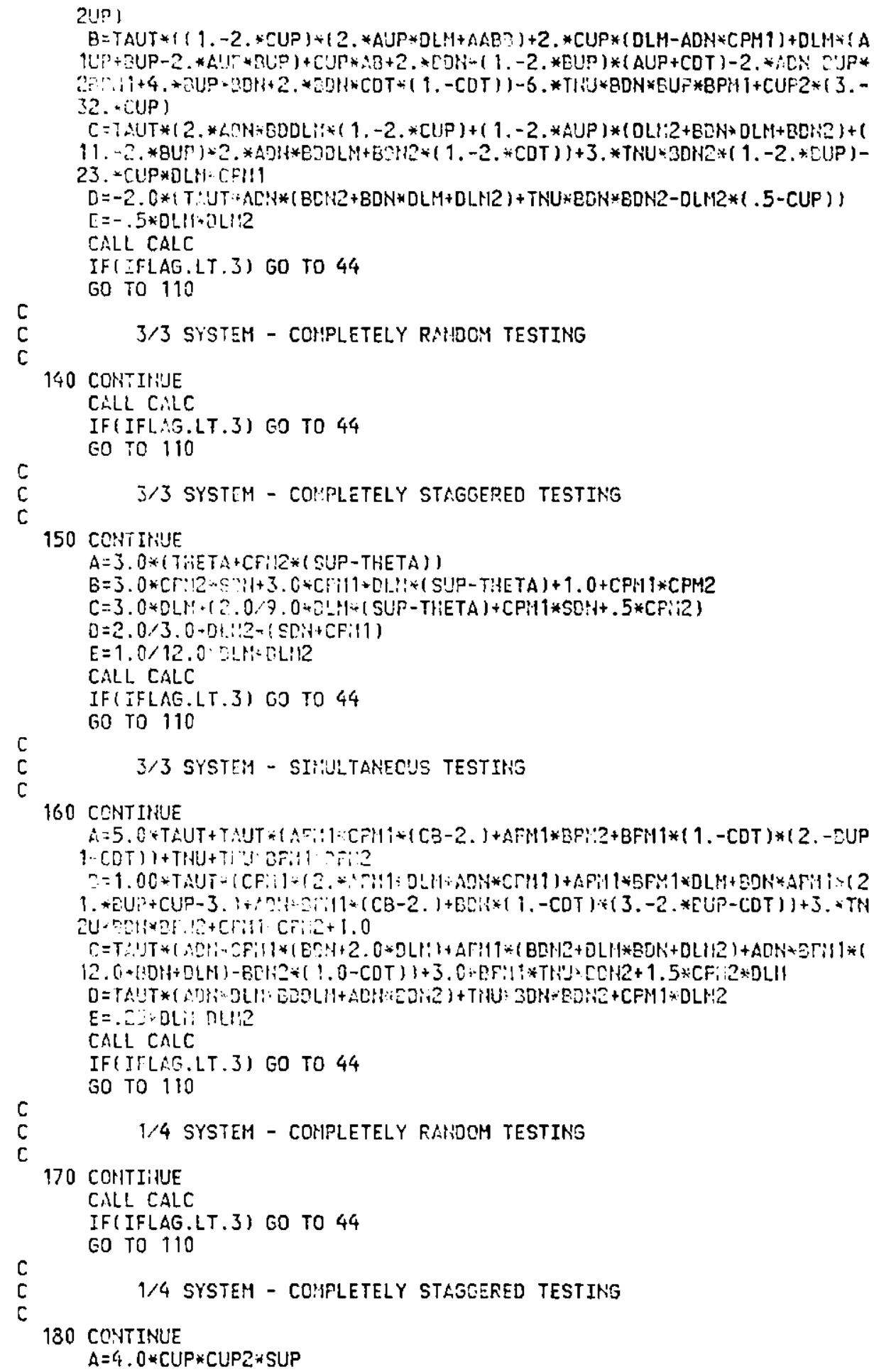




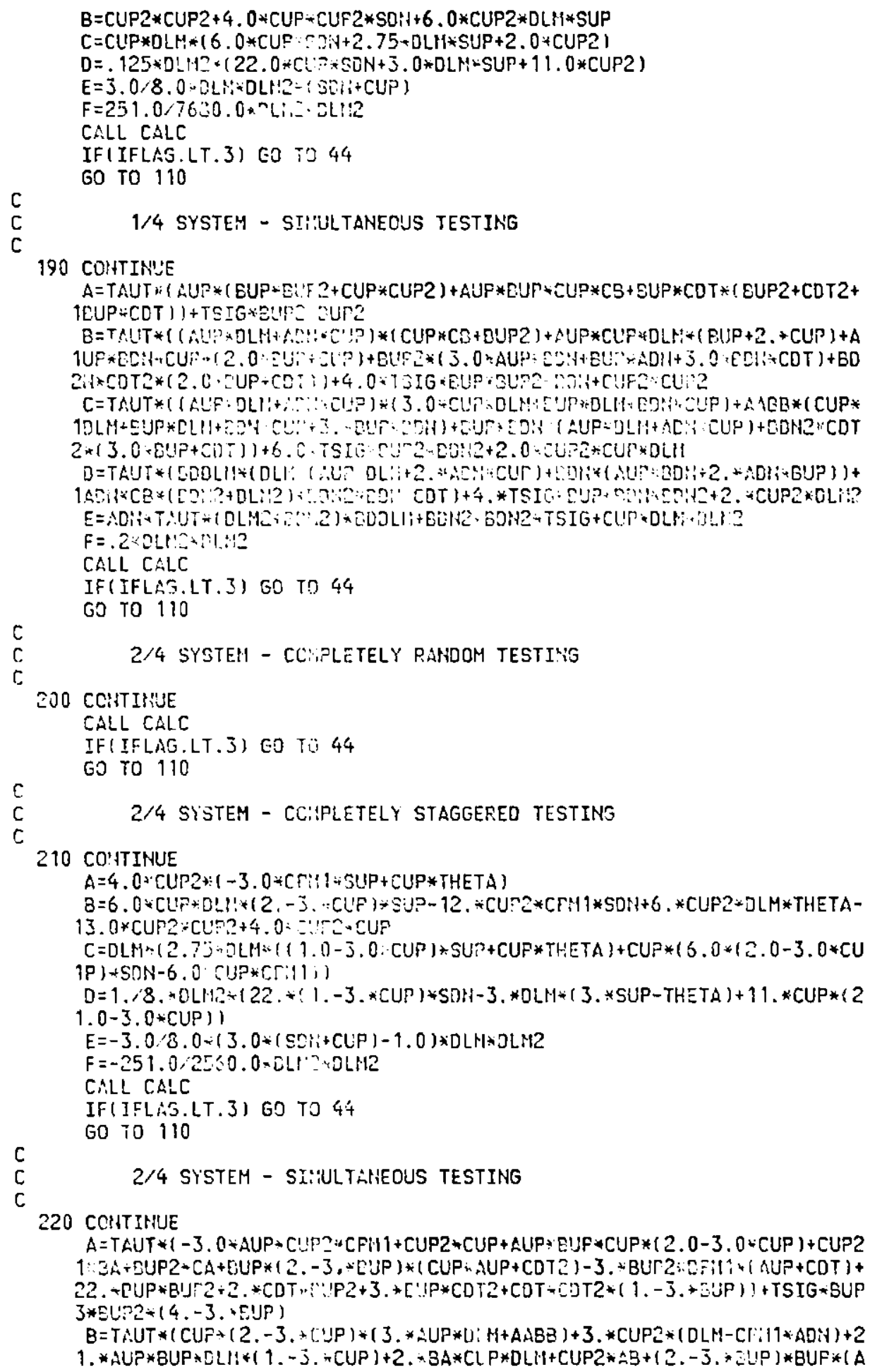




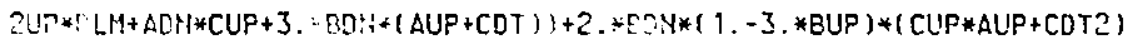

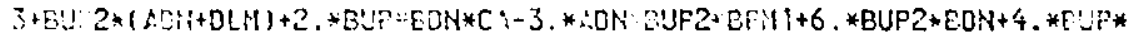

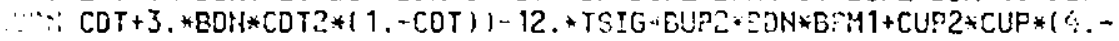
$\because \therefore$ U UP)

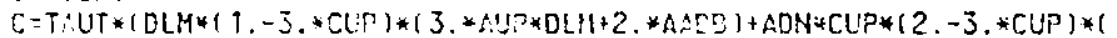

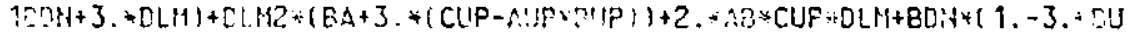

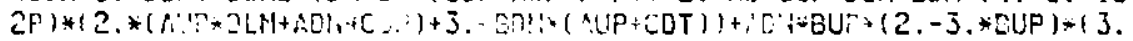

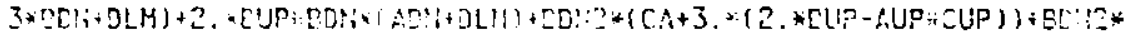

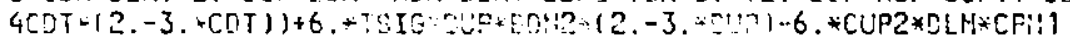

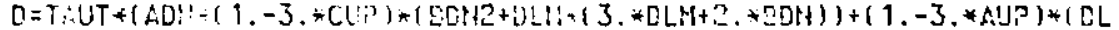

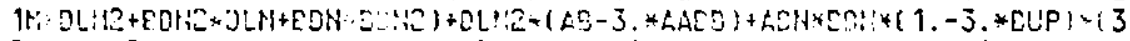

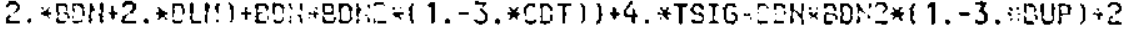
3. *CUT:DLM $2 * i 2 .-3 . * C U P)$

$E=-3 . * A O H * T A \cup T * E D D L M *(B D * 12+D L M ?)-3 . * T S I G * E D H 2 * E D N 2+D L M * D L H 2 *(1,-3$. $1 \times C U: 1$

$F=-3.0 / 5.0 * D L N 2 * D L M 2$

CALL CALL

IF(IFLAG.LT.3) GO TO 44

G) TO 110

C

230 CONTIHIJE

C:LL CALC

IF (IFL:SG.LT.3) GO TO 44

c

EO TO 110

C

240 CCHTIR:UE

3.4 SYSTEM - CC:IPLETELY STAGGERED TESTING

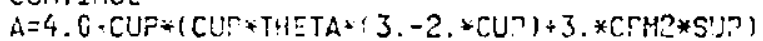

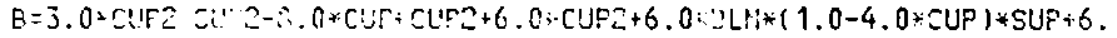

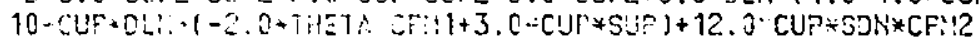

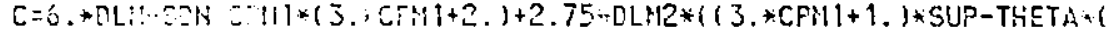

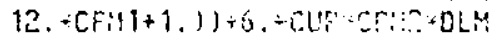

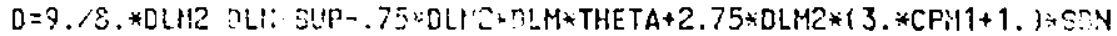

$1+11 ., 3 . *(3 . * C r: 19+2):. 25119+21: 12$

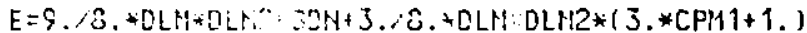

$\mathrm{F}=251.12530 .+0 \mathrm{LH} / \mathrm{OLI}: ?$

C..LL CALC

IF(IFIAG.LT.3) GO TO 44

ES TO 110

C

C

250 CC:TTHERE

$3 / 4$ SYSTEII - SIMJLTANEOUS TESTING

$A=T A \cup T *(3, A A U F * C U P+C F: 12+2, * C U F ? *(2,-C U P)+A U P * C U P * C U F *(3, * C B-3,1+1 B$

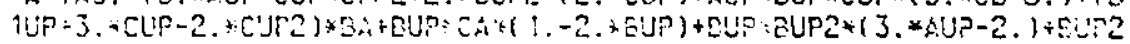

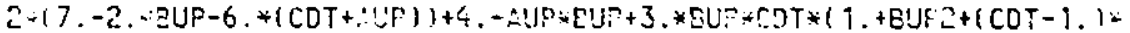

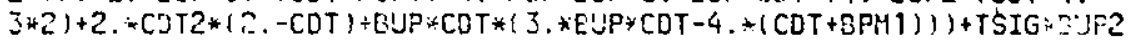
$4 *\left(3 . * \operatorname{EUP2}-3 . \mathrm{U}^{2}+6.\right)$

$B=T: U T *(3, * A C, 1, C U P * C F: 2+(3, * C(1 P-1) * C F M 1 *,(3, * A U P * D L M+A A C B)-2, * C U P *$

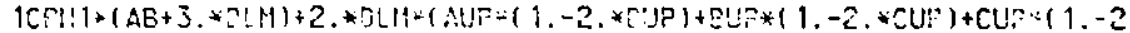

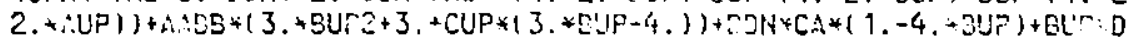

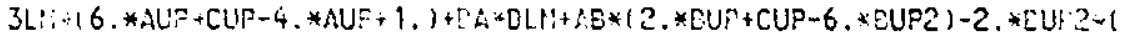

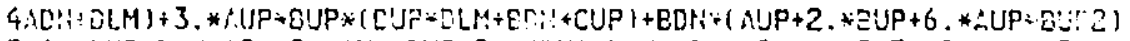

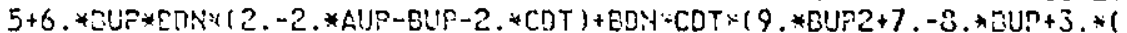
6 CDT $-1.1 * * 2)+2 . * E D i * C D T 2 *(3 . * S U P-2.1)+12 . * E U P * B D N * T S I G * B F M 2+C U P 2 *(3$ 7. $*$ CUP2 $-8 .+C U P+6.1$

$C=T A U T *(3 . * A C H *-D L M * 13 . * C U P-1) * C F H 1+.O L M 2 * 13 . * A U P * B U P-2 . * B A-6 . * C U ?+$ 
14. $)+(3 . * C U P-2) * O L M+.(2 . * A A B S+3 . * \angle U F * D L M)+A D N * B D N * C U F *(3 . * C U P-8)+$, $2 D N=B D H * 19 . * E ! J P 2+1).+3 D H *(1 .-4 . * B(L P) *(4 . * A D H * J L H)+\triangle A C 8 * 33 . *(B U P * D L M$

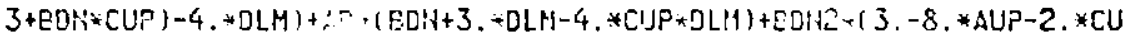

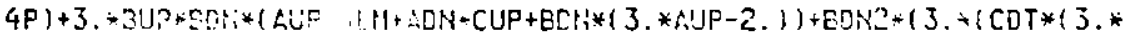

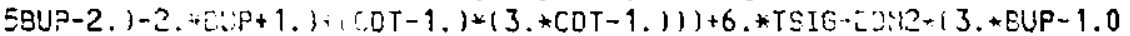
$6)+E F M 1+6, * C L P: D L M+C .: 3$

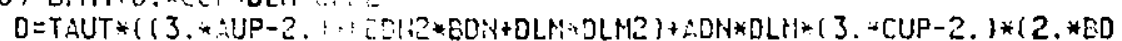

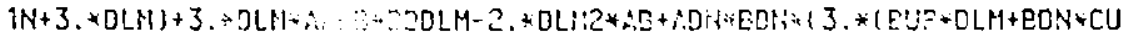

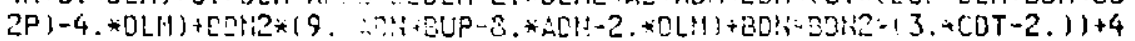

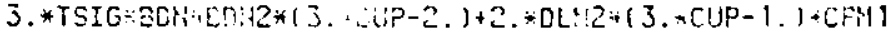

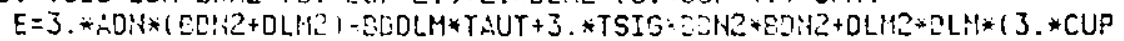
$\{-2$.

$F=3.15 \cdot 2 \mathrm{DLH} 2 * \mathrm{DLM} 2$

CALL CALC

IF (IFLAG.LT. 3) GO TO 44

C

GO TO 110

C

250 COMTITIUE

CALL CALC

IF IFLAG.LT.3) GO TO 44

C

6C. TO 110

C 4/4 SYSTEM - CCI:PLETELY STAGGERED TESTING

270 COHTINUE

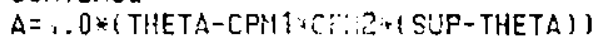

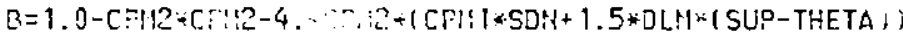

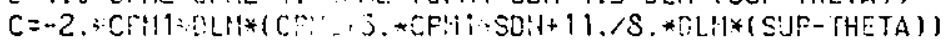

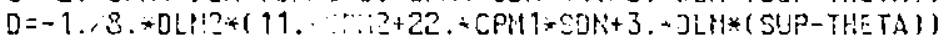

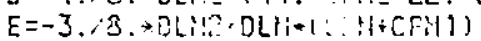

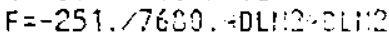

CALL. CALC

IF(IFLSG.LT.3) GO TO 44

$c$

GO TO 110

C 4/4 SYSTEM - SIMULTAMEOUS TESTING

280 CONTINUE

$A=7, * T A U T-T A I J T *(A F H I)+C F M 1 * C F: 12+8 P H 1 *(A F: 11 * C P H 2+(C O T-1) * * 3)+,B P M 2 * 1$

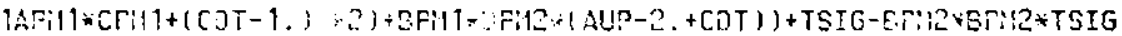

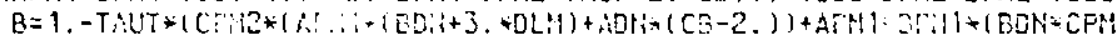

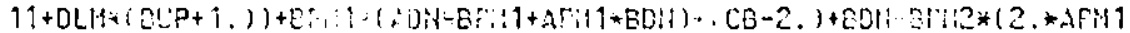

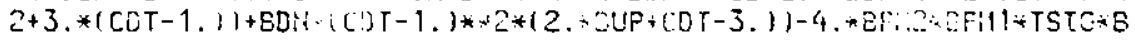
SOH-CFit? + CFH?

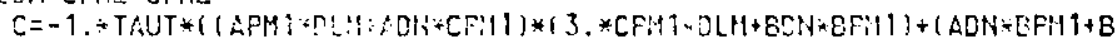

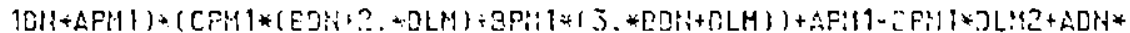

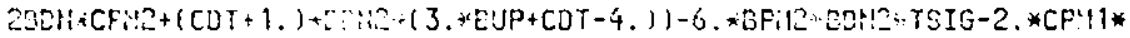
JCFHI $\div$ OL:I

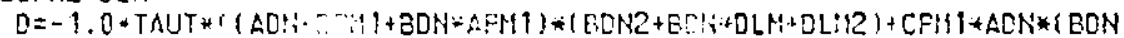

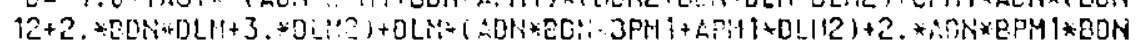

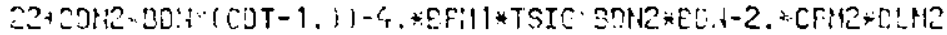

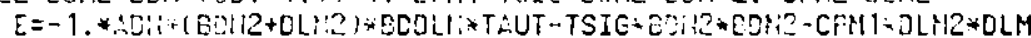

$F=-1,0,5.0 * 01.112 * 2 L 1$.

CALL CALC

IFIIFLAG.LT.3) GO TO 44

GO TO 110

C

C WRITE ERROR MESSAGE AHIO STOP IF INPUT CRITERIA NOT MET. 


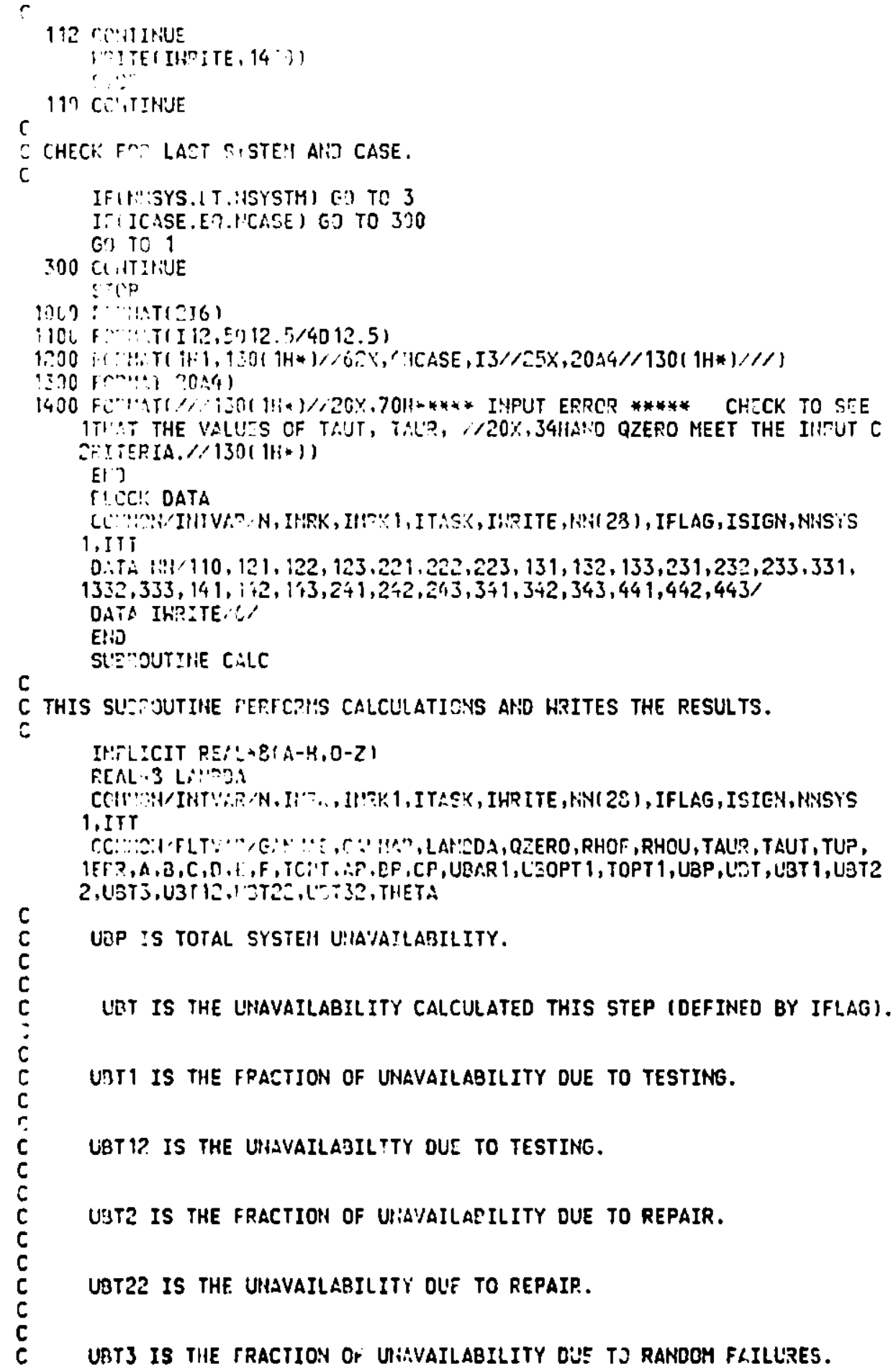




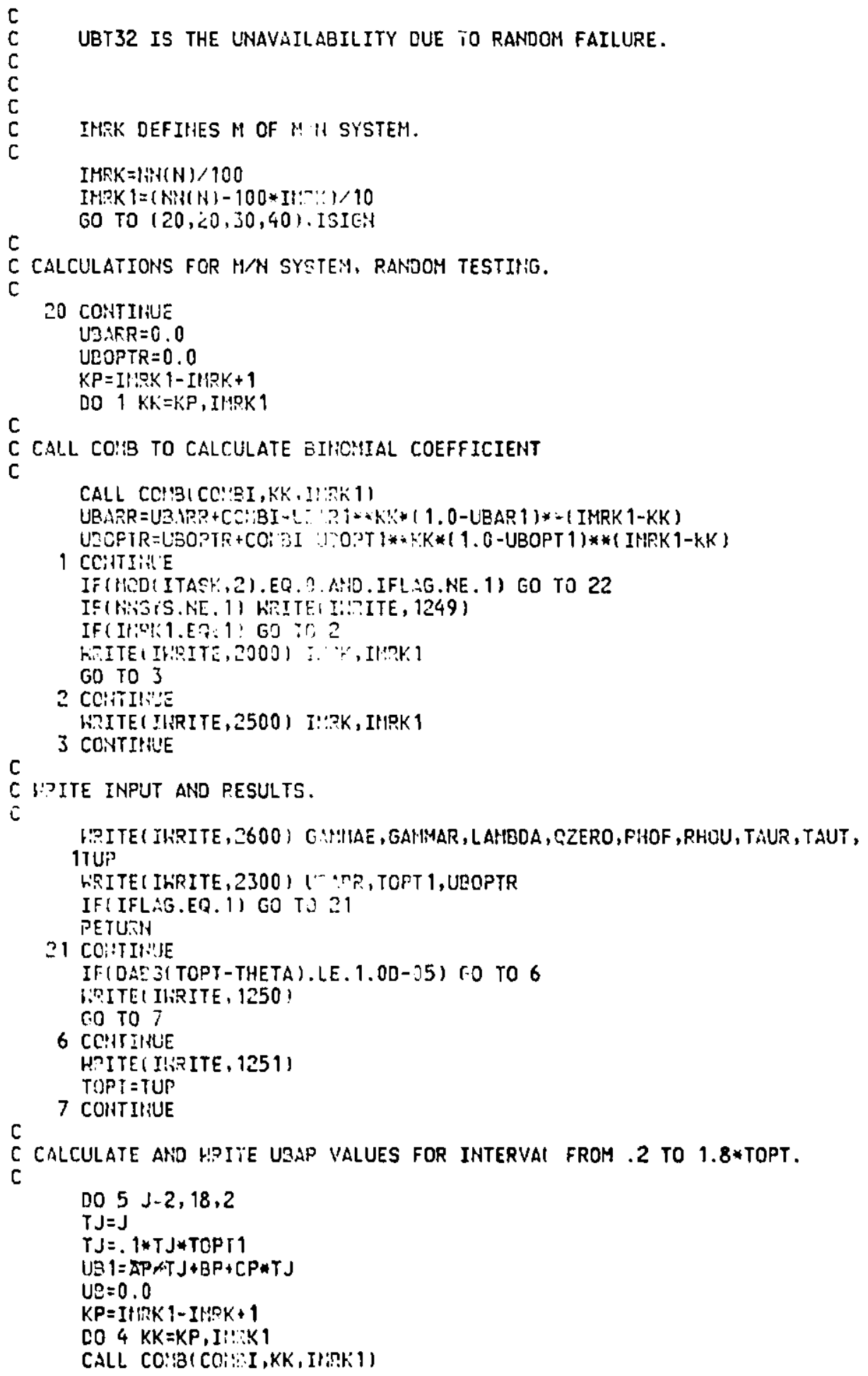




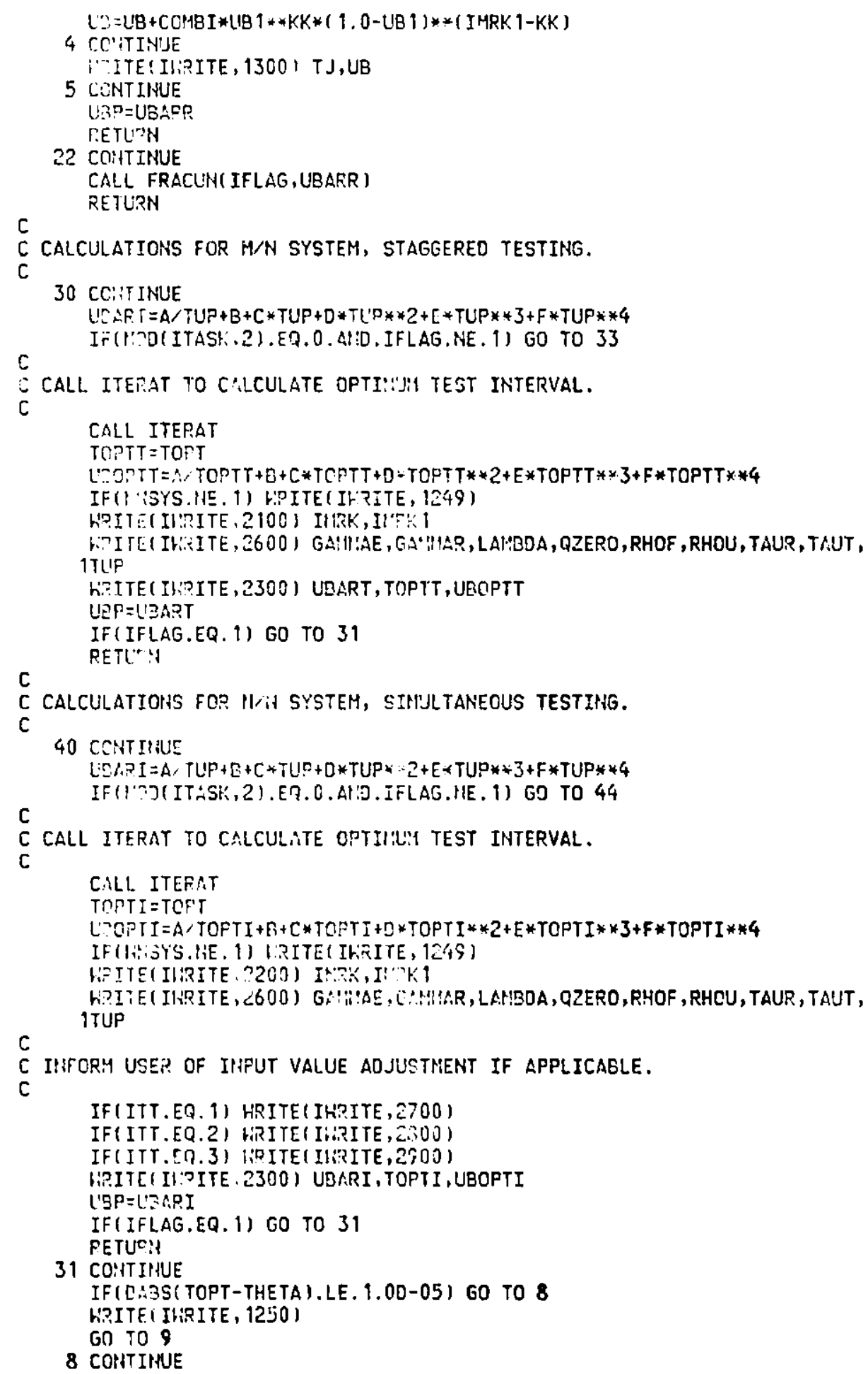




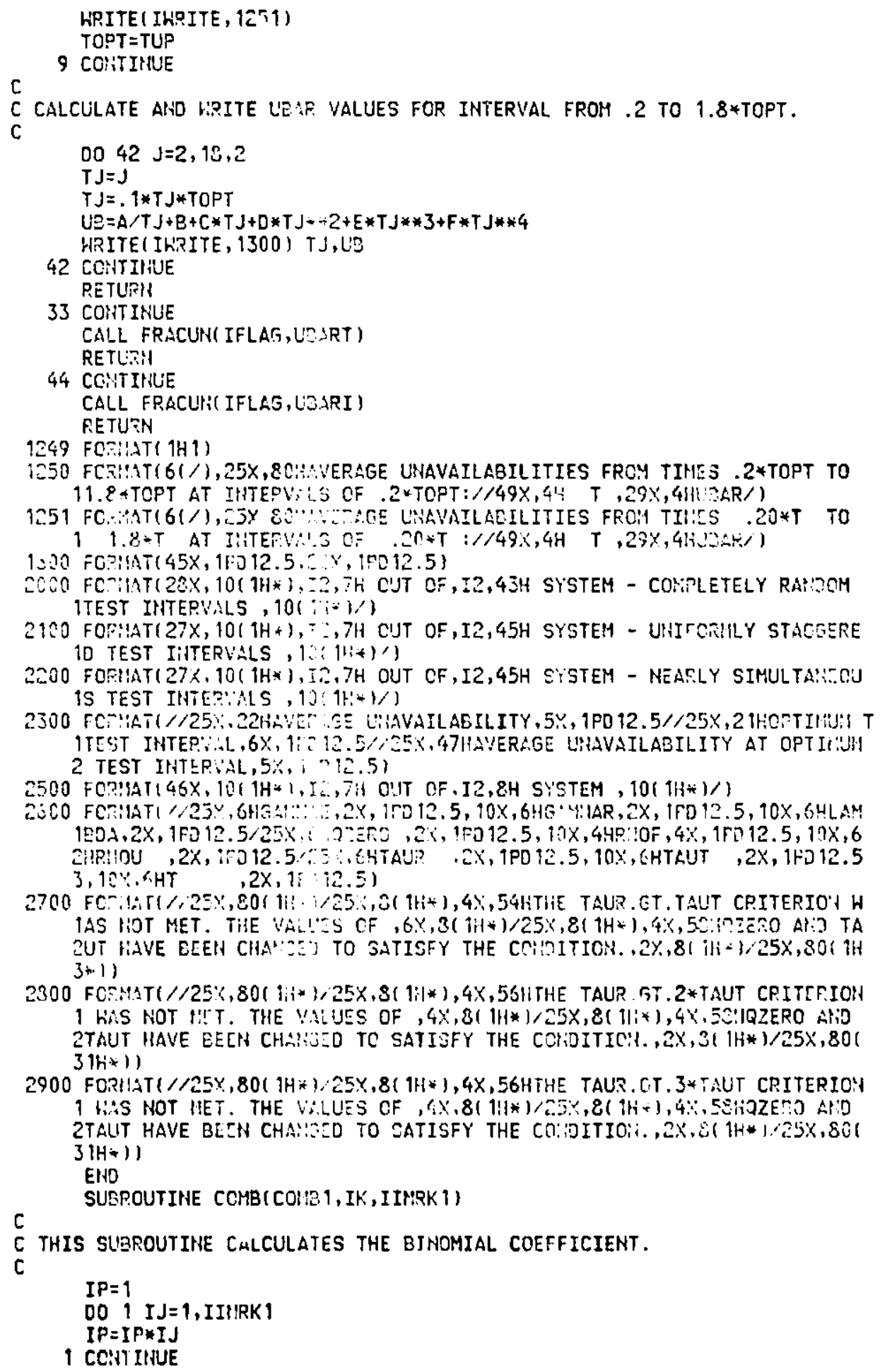




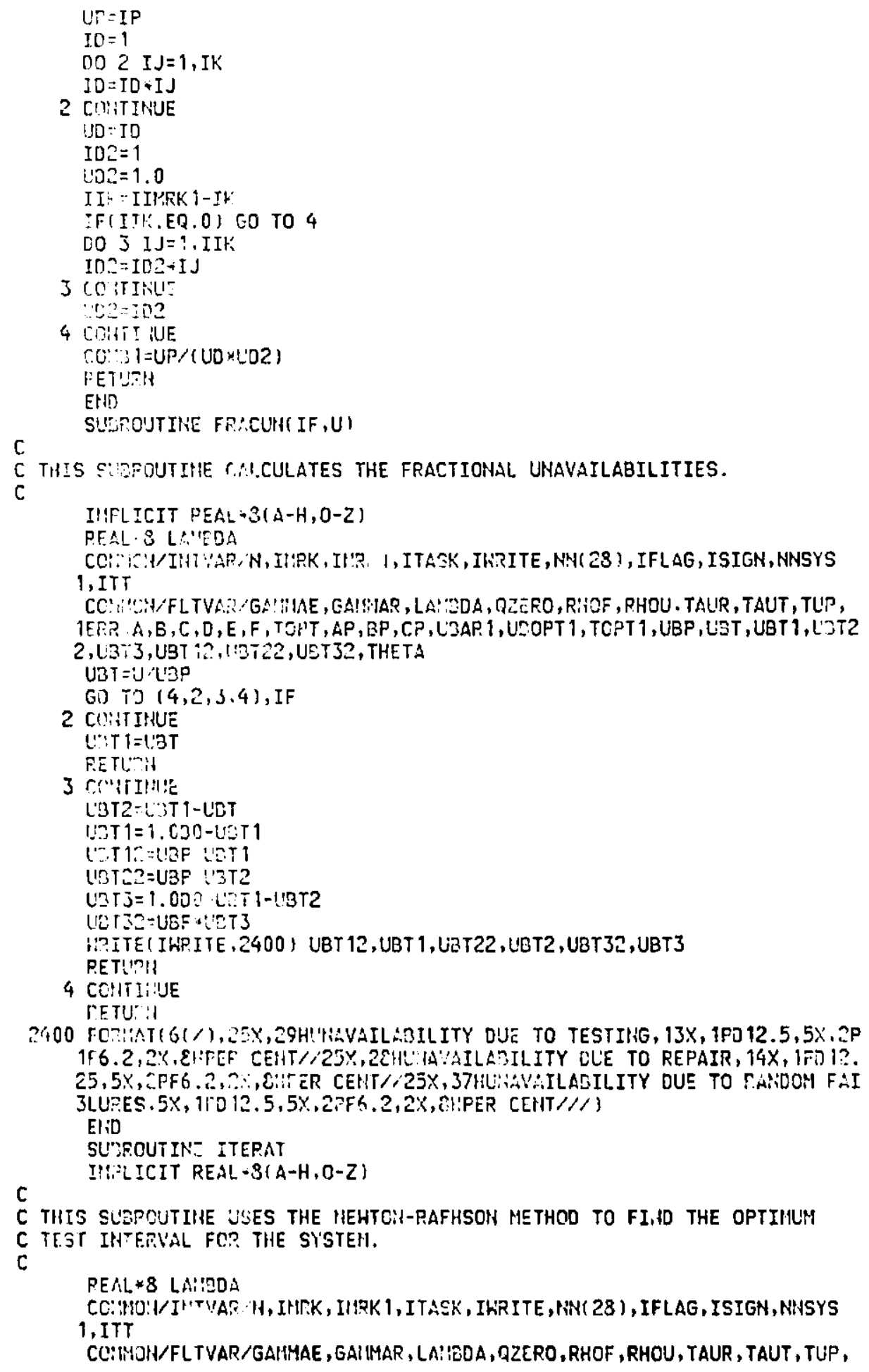


1EFF, A , B,C, D, E , F , TOPT , AP , BP, CP, UBAR 1, UBOPT 1, TOPT 1, UBP, UBT , UBT 1, U3T2 2, UST3, LBT 12, UBT22, UBT32, THETA

TOLD = TOPT 1

1 COKTIU:LE

$F X=\{. C$ F $* T O L O * 5+3.0 * E *$ TULD $0 * 4+2.0 * 0 *$ TOLD $* 3+C *$ TOLD $* 2-A$

$F X F=20.0 * F * T C: D * * 4+12.0: 5 * T O L D * 3+6.0 * D * T O L D * 2+? .0 * C * T O L D$

$T T=T O ! D-F X / F X P$

IF (TT.LE.THETA) GO TO 3

IFIURES(TT-TOLD).LE.(ENP..TT)\} GO TO 2

TOL:?:TT

(10 1

a Contel:Je

TUंग =TT

CO TO 4

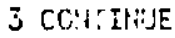

TOPT = THETA

4 CrititiUe

RETU?N

EEI:D 
$\underline{\text { IC } f_{\mathrm{R}} \mathrm{RUS} \text { Input and Output }}$

Presented is a sample computer run of ICARUS for two systems: a $1 / 3$ systrm tested usid the uniformly staggered testing scheme and a $2 / 4$ sysem using tne uniformly staggered testing scheme. The task flag is set to calculate the average unavailability as a function of test interval and also to calculate the fractions of the average unavailability due to testing, repair, and random failures.

There were eight input cards, and they appeared in the following order:

Card 1, NCASE $=1$, column 6

Card 2, ITASK $=2$, column 6

Card 3, ITITLE = ICARUS Documentation Run, columns 29-52

Card 4, NSYSTM $=2$, column 6

Card 5, 1MARK $=132$, columns $10-12$

GAMMAE $=1.0 \mathrm{D}-03$, columns $18-24$

GAMMAR = 1.CD-03, columns 30-36

LAMBDA $=3.0 \mathrm{D}-07$, columns $42-48$

Q7.ERO = 1.0D-01, columns 54-60

RIIOF $=1.0 \mathrm{D}-03$, columns $66-72$

Card 6, RHOU $=1.0 \mathrm{D}-03$, columns $6-12$

TAUR $=7.0$, columns $22-24$

TAUT $=3.0$, columns $34-36$

$\Gamma \cup P=720.0$, columns 44-48

Card 7, IMARK = 242, columns $10-12$

Rest of Card 7 is the same as Card 5.

Card 8 is the saine as Card 6.

The output is arranged in the following manner. The case title is printed first with earh of the systems to be studied following. The system title is printed, as are the values that ICARUS uses to do the calculations. (These may be djfferent from the input if ICARUS makes internal modifications to the input.) Next, ICARUS prints the average unavailability, the optimum test interval, and the average unavailability at the optimum test interval.

If the task flag is set, the code will print the average unavailability for values of the test interval that range from 0.2 to 1.8 times the optinum. The same flag will calculate and print the fractional unavailabilities. 
ICARUS UUCUMENTATION RUN

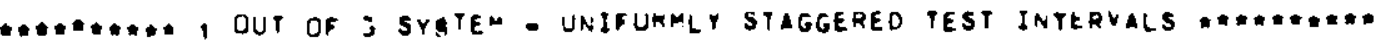

\begin{tabular}{|c|c|c|c|c|c|}
\hline $\begin{array}{l}\text { GIMMAE } \\
\text { GIERO } \\
\text { TAUR }\end{array}$ & $\begin{array}{l}1.00 n n 00=03 \\
1.000000=01 \\
7.00000000\end{array}$ & $\begin{array}{l}\text { GAMEAR } \\
\text { REOF } \\
\text { TAUT }\end{array}$ & $\begin{array}{l}1.000000-03 \\
1.000000=03 \\
3.00000000\end{array}$ & $\begin{array}{l}\text { LAMEDA } \\
\text { BHOU } \\
T\end{array}$ & $\begin{array}{l}1.00000 u=07 \\
1.0000 u v=03 \\
7.200000\end{array}$ \\
\hline AvERAGE & UNAVAILABILITY & 1.529800 & & & \\
\hline OPTImUM & TEST INTERVAL & 1.331920 & & & \\
\hline
\end{tabular}

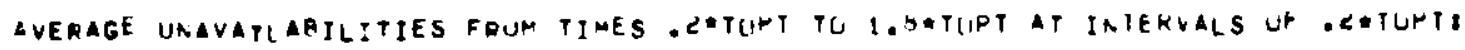

个

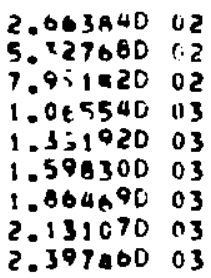

UUAR
UNAYALAEILITY OUE TO TESTING UNAVAILAOILITY DUE TO REPAIR

UNAVAILABILITY OUE TO RANDOM FAILURES
$5.529550-09$

$2.871250 \cdot 10$

$0.482100-09$
36.14 PEN CENT

1.96 PEK CENT

61.98 PEK CENT 


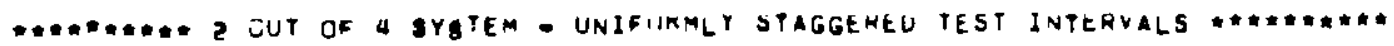

\begin{tabular}{|c|c|c|c|c|c|}
\hline $\begin{array}{l}\text { GAMNAE } \\
\text { OZERO } \\
\text { TAUP }\end{array}$ & $\begin{array}{l}1.000000=03 \\
1.000000=01 \\
7.00000000\end{array}$ & $\begin{array}{l}\text { GAMMAK } \\
\text { RHOF } \\
\text { TAUT }\end{array}$ & $\begin{array}{l}1.000000-03 \\
1.000000-03 \\
3.00000000\end{array}$ & $\begin{array}{l}\text { LAMBLA } \\
\text { RHOU } \\
T\end{array}$ & $\begin{array}{l}3.000000=07 \\
1.000000=03 \\
7.200000 \text { 0 }\end{array}$ \\
\hline AVERAGE & UNAVAILAFILITY & 0.10159110 & & & \\
\hline UPTIMUM & TEST JNTERVAL & $1.1+1+30$ & 3 & & \\
\hline
\end{tabular}

AVERAGE UNAVATLABILITIES FPUM PIMES .

\section{T}

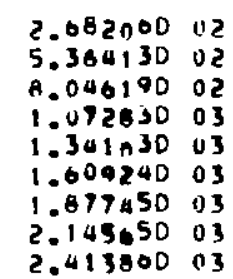

USAR

$9.457040-1.8$
$0.757280=08$

$6.002060-C 8$

$5.738740-08$

$5.070780-08$

$5.719400-08$

$5.825200-08$

$5.973530-08$

$0.152970-78$
L'NaVAILABILITY DUE TO TESTING

unavaILaBILITY OUE TO REPAIK

UNAVAILABILITY DUE TO RANDAM FAILURES

$\begin{array}{rr}2.207320-0 E & 35.82 \text { PEK CENT } \\ 1.146100 .09 & 1.80 \text { PEN CENT } \\ 3.839050-08 & 02.32 \text { DEK CENT }\end{array}$




\section{APPENDIX F}

Data Base for $\rho_{u}, \rho_{f}, \gamma_{e}$, and $\gamma_{r}$ Type Failures

The following reported occurrences were used as the bases for the values of the parameters $\rho_{u}, \rho_{f}, \gamma_{e}$, and $\gamma_{r}$ in the auxiliary-feed-water-system analysis of Chapter $V$.

Failure Type: ou

Plar.t: Zion Unit 2 Docket No.: 50-304-183

Event: Three Related Abnormal Occurrences with Diesel Generation

Date: February 17, 1974

Cause: The unit was operating at $25 \%$ power with two auxiliary feedwater pumps inoperable. During the daily testing of the third pump, the standby ac power-supply Diesel generator started, but tripped out on overspeed. Due to an operator error, the synchro-speed setter had been set on 13.5 instead of 12. After it was reset, the Diesel operated properly. Then the output breaker would not close on the bus so that the $D / G$ control panel was latched closed. A loose screw was lodged in the pivot. The screw was removed. The lapsed time to correct these defects excecded the $4-\mathrm{h}$ limit of operating without an AFWS pump by $2.7 \mathrm{~h}$.

Failure Type: $\rho_{f}$

Plant: Turkey Point Unit No. 3 Docket No.: 50-250-230

Event: Malfunction of Auxiliary Feedwater Pump:;

Date: May 8, 1974

Cause: All three auxiliary feedwater pumps had been tested satisfactorily on May 7, 1974, according to the operation procedure OP7304.1, which is the monthly test. After the test, the pump packing was adjusted on the $A$ and $B$ pumps. Apparently, the packing was tightened with the pumps secured, and the packing was tight enough to prevent the pumps fi $\mathrm{sm}$ starting. The pumps were not tested for operability after the packing was tightened.

Failure Type: $P_{u}$

Plant: Prairie Island

Nuclear Gencrating Plant I Docket No.: 50-282-274

Event: Failure of No. 12 Auxiliary Feedwater Pump to Start

Date: August 13,1974

Cause: During testing with the reactor at $100 \%$ power, an electric auxiliary-feedwater-system pump failed to start. During maintenance 
on August 6, 1974, someone inadvertently opened the breaker for the auxiliary lube oil pump. This action prevented the No. 12 auxiliary feedwater pump from starting due to the lube-oil pressure interlock.

Failure Type: Pu

Plant: H. B. Robinson Unit No. 2 Docket No.: 50-261-454

Event: Overspeed Trip of Steam-driven Auxiliary Feedwater Pump

Date: November 19, 1974

Cause: Investigation revealed the woodward governor manual setting was out of adjustment. It could not be determined if the setting was inadvertently moved after a previous test or changed by noncognizant personnel working in the area.

Failure Type: $\rho_{f}$

Plant: Oconee 3 Docket No.: 50-287-268

Event: Inoperable Emergency Feedwater Pump

Date: April 30, 1975

Cause: Maintenance was performed on the emergency feedwater pump to repair a leaking packing gland on April 19, 1975. The packing was apparently set too tight, and the pump was returned to service without verifying operability as is required by procedure.

Failure Type: i

Plant: Trojan Nuclrar Plant Docket No.: 50-344-249

Event: Diesel-driven Auxiliary-feedwater-pump Failure to Start

Date: Janliary o, 1976

Cause: This occurrence was caused by a change in engine conditions (oil and water temperatures, etc) from those under which the governor had last been aligned.

Failure Type: iu

Plant: Trojan Nuclear Plant Docket No.: 50-344-387

Event: The Turbine-driven Auxiliary Feedwater Pump Failed to Automatic Start

Date: October 19,1976

Cause: The trip throttle valve (MO 3071) was not properly reset by an auxiliary operator for an automatic start. 
Failure Type: $\rho_{u}, v_{e}, \gamma_{r}$

Plant: Millstone Unit 2 Docket No.: 50-336-539

Event: Pump Inlet Steam Valve Fails to Open

Date: July 24,1976

Cause: The cause of the valve-shaft shearing prohibiting the valve from opening was discovered to be improper torque switch setting (reported October 22, 1976).

Failure Type: $v_{e}, v_{r}$

Plant: Zion Unit 1 Docket No.: 50-295-324

Event: An Auxiliary Feedwater Pump Found Inoperable

Date: June 6, 1974

Cause: On June 6, 1974, at $2115 \mathrm{~h}$ with the unit 1 reactor operating at $75 \%$ power level and the steam-generator load at $750 \mathrm{MW}$, the shift supervisor, during a tour of the plant, found the overspeed trip valve on the 1 A steam-turbine-driven auxiliary feedwater pump in the tripped position. The trip valve was reset, and an attempt was made to run the pump. The pump started normally, but water accumulation in the turbine exhaust pipe caused an excessive water hammer that broke one of the exhaust pipe hangers. Because of this, the $1 \mathrm{~A}$ auxiliary feedwater pump was shut down and declared inoperable pending repairs.

Failure Type: $\gamma_{e}, \gamma_{\mathbf{r}}$

Plant: Zion Unit 1 Docket No.: 50-295-606

Event: Auxiliary Feedwater Pump Fails to Start

Date: March 5, 1976

Cause: The pump had been started and stopped several times to maintain steam-generator pressure. Because of this, moisture accumulation was highe: than normal in the turbine and moisture could not drain off properly. The pump thus became waterbound and failed to start. 


\section{ACKNOW LEDGMENTS}

This refort summarizes results obtained under a contract between Argonne National Laboratory and the University of Lowell. We would like to thank Dr Gilbert J. Brown as well as all the other members of the Department of Nuclear Engineering, University of Lowell, for helpful comments and discussion during the work. We also thank all our friends at Argonne National Laboratory, especially Jim Cahalan and the rest of the members of the Reactor Analysis and Safety Division, who were always ready to provide help and encouragement.

\section{REFERENCES}

1. A. E. Greene and A. J. Bourne, Reliability Technology, Wiley-Interscience, London, p. 272 (1972).

2. E. Dressler and H. Spindler, the Unavailability of Standby Systems as a Function of Test Strategy and Repair Time (in German), MRk 144, Technica1 University at Munich (1975).

3. J. P. Signoret, Availability of a Periodically Tested Standby System, NUREG/TR-0027 (1976).

4. G. E. Apostolakis and P. P. Bansal, Effect of Human Error on the Availability of Periodically Insrected Redundant Systems, IEEE Trans. Reliab. R-26(3), 220 (1977).

j. W. E. Vesely and F. F. Goldberg, Time Dependent Unavailability Analysis of Nuclear Safet? Systems, IEEE Trans. Reliab. R-26(4), 257 (1977).

6. W. E. Vesely and F. F. Goldberg, FRANTIC--A Computer Code for Time L'ependent Unavailability Analysis, NUREG-0193 (1977).

7. J. K. Vaurio, Unarailability of Components with Inspection and Repair, Nucl. Eng. Des. (in press).

8. J. K. Vaurio, "Response Surface Tec:iniques Developed for Probabilistic Analysis of Accident Consequences," Proc. ANS Topical Meeting, Los Angeles, p. II.2 (May 1978).

9. M. R. Rosenthal, Numerical Methods in Computer Programing, Richard D. Irwin, Inc., Homewood, Ill., pp. 236-237 (1966).

10. Reactor Safoty Study: An Assessment of Accident Risis in U. S. Commercial Nuclear Power Plants, WASH-1400, USNRC (Oet 1975).

11. J. D. Millan and G. E. Edison, Comnon Cause FaiZure Experience in PWR Auriliary Feedwater Syotems for Large Rreeder Reliability Analysis, WARD-SR-3045-5 (Aug 1978).

12. V. Joksimovic et al., "HTGR Risk Assessment Study," Proc. Int. Conf. Nuclear Sustem Reliability Engineering and Risk Assessment, Gatlinburg, Tenn. p. 180 (1977). 